\title{
Associative white matter connecting the dorsal and ventral posterior human cortex
}

\author{
Bullock, D., Takemura, H., Caiafa, C. F., Kitchell, L., McPherson, B., Caron, B., and Pestilli, F.
}

\author{
Affiliation \\ 1. Daniel Bullock. Department of Psychological and Brain Sciences, Program in Neuroscience, Indiana University Bloomington, \\ 1101 E 10th Street, Bloomington, Indiana USA 47405; Center for Information and Neural Networks (CiNet), National Institute of \\ Information and Communications Technology. Email: dnbulloc@indiana.edu \\ 2. Hiromasa Takemura. Center for Information and Neural Networks (CiNet), National Institute of Information and Communication \\ Technology, and Osaka University; Graduate School of Frontier Biosciences, Osaka University, 1-4 Yamadaoka, Suita-shi, \\ Osaka Japan 565-0801. Email: htakemur@nict.go.jp \\ 3. Cesar Caiafa. Department of Psychological and Brain Sciences, Indiana University Bloomington, $1101 \mathrm{E} 10$ th Street, \\ Bloomington, Indiana USA 47405. Instituto Argentino de Radioastronomía (CCT-La Plata, CONICET; CICPBA), CC5 V. Elisa, \\ ARGENTINA, 1894. Facultad de Ingeniería - Universidad de Buenos Aires, Buenos Aires, ARGENTINA, C1063ACV. Email: \\ ccaiafa@gmail.com \\ 4. Lindsey Kitchell. Department of Psychological and Brain Sciences, Department of Cognitive Science, Indiana University \\ Bloomington, 1101 E 10th Street, Bloomington, Indiana USA 47405; Email: kitchell@umail.iu.edu \\ 5. Brent McPHerson. Department of Psychological and Brain Sciences, Department of Cognitive Science, Indiana University \\ Bloomington, 1101 E 10th Street, Bloomington, Indiana USA 47405; Email: bcmcpher@umail.iu.edu \\ 6. Bradley Caron. Program in Neuroscience, Vision Science, Indiana University Bloomington,702 N Walnut Grove St, Bloomington, \\ Indiana USA 47405; Email: bacaron@iu.edu \\ 7. Franco Pestilli. Department of Psychological and Brain Sciences, Engineering, Computer Science, Programs in Neuroscience \\ and Cognitive Science, School of Optometry, and Indiana Network Science Institute, Indiana University Bloomington, $1101 \mathrm{E}$ \\ 10th Street, Bloomington, Indiana USA 47405. Email: franpest@indiana.edu
}

Contribution D.N.B. and F.P. conceptualized and performed analyses. H.T. and C.C. provided data curation and software. D.N.B., H.T., C.C., and F.P. wrote the manuscript. L.K., B.M., and B.C. provided validation and software.

Competing interests The authors declare no competing financial interests.

Corresponding authors Franco Pestilli franpest@indiana.edu

\begin{abstract}
Historically, the primary focus of studies of human white matter tracts has been on large tracts that connect anterior to posterior cortical regions. These include the superior longitudinal fasciculus (SLF), the inferior longitudinal fasciculus (ILF), and the inferior fronto-occipital fasciculus (IFOF). Recently, more refined and well understood tractography methods have facilitated the characterization of several tracts in the posterior of the human brain that connect dorsal to ventral cortical regions. These include the vertical occipital fasciculus (VOF), the posterior arcuate fasciculus (pArc), the temporo-parietal connection (TP-SPL), and the middle longitudinal fasciculus (MdLF). The addition of these dorso-ventral connective tracts to our standard picture of white matter architecture results in a more complicated pattern of white matter connectivity than previously considered. Dorso-ventral connective tracts may play a role in transferring information from superior horizontal tracts, such as the SLF, to inferior horizontal tracts, such as the IFOF and ILF. We present a full anatomical delineation of these major dorso-ventral connective white matter tracts (the VOF, pArc, TP-SPL, MdLF). We show their spatial layout and cortical termination mappings in relation to the more established horizontal tracts (SLF, IFOF, ILF, Arc) and consider standard values for quantitative features associated with the aforementioned tracts. We hope to facilitate further study on these tracts and their relations. To this end, we also share links to automated code that segments these tracts, thereby providing a standard approach to obtaining these tracts for subsequent analysis. We developed open source software to allow reproducible segmentation of the tracts: https://github.com/brainlife/Vertical_Tracts. Finally, we make the segmentation method available as an open cloud service on the data and analyses sharing platform brainlife.io. Investigators will be able to access these services and upload their data to segment these tracts.
\end{abstract}

Keywords: diffusion imaging; white matter; historical; tractography; dorsal and ventral streams; computational neuroanatomy 


\section{Acknowledgements}

This research was supported by NSF IIS-1636893, NSF BCS-1734853, NIH NCATS UL1TR002529, a Microsoft Research Award, Japan Society for the Promotion of Science (JSPS) KAKENHI (JP17H04684, JP15J00412) and the Indiana University Areas of Emergent Research initiative "Learning: Brains, Machines, Children." In part by NIH NCATS UL1TR002529 to F.P.. D.N.B. and B.M. were partially funded via NIH NIMH 5 T32 MH103213 to B. Hetrick and B. D'Onofrio. We thank Soichi Hayashi, Steven O'Riley, David Hunt and Aman Arya for contributing to the development of brainlife.io, Craig Stewart, Winona Snapp-Childs, David Hancok and Jeremy Fischer for support with jetstream-cloud.org (NSF ACl-1445604). Data were provided in part by the Human Connectome Project, WU-Minn Consortium (Principal Investigators: David Van Essen and Kamil Ugurbil; 1U54MH091657) funded by the 16 NIH Institutes and Centers that support the NIH Blueprint for Neuroscience Research; and by the McDonnell Center for Systems Neuroscience at Washington University. Data were provided in part by Brian Wandell (Stanford University; https://purl.stanford.edu/bb060nk0241). Thanks to Sophia Vinci-Booher for comments on early versions of the manuscript. Thanks also to Josh Faskowitz for help with software.

\section{Compliance with ethical standards.}

Conflicts of interest:

Authors declare no conflicts of interest.

Research involving human participants and/or animals:

Data collection was approved by the respective Institutional Review Boards (IRBs) of the University of Washington, Saint Louis (HCP dataset) and Stanford University (STN dataset).

Informed consent.

All participants provided written informed consent to participate in the project 


\section{INTRODUCTION}

Scientific investigations of brain anatomy and connections, using post mortem methods, have been ongoing for more than two centuries (Obersteiner 1890; Gray 1918; Catani and de Schotten 2012). In this time, a number of major white matter tracts have been established in the human brain (Mori et al. 2005; Schmahmann et al. 2007; Catani and de Schotten 2012). Typically, these major tracts conform to a rule proposed by Theodore Meynert (Charcot 2016) which holds that the major associative tracts follow an anterior-posterior orientation. This is reflected in many historical depictions of white matter anatomy (Figure 1a (Edinger 1885, 1893)). This canon of anterior-posterior oriented white matter tracts was established primarily by post mortem anatomical dissections and camera lucida drawings (Wernicke 1881; Obersteiner 1890; Gray 1918; Yeatman et al. 2014). Hereafter, we focus our analysis on four of these major horizontal tracts and refer to them as canonical in virtue of their conformity with Meynert's principle. These canonical tracts include the superior longitudinal fasciculus (SLF), the arcuate fasciculus (Arc), the inferior longitudinal fasciculus (ILF), and the inferior fronto-occipital fasciculus (IFOF). Work using post mortem anatomical dissection continues to provide fundamental insights into the structure of these and other white matter tracts (De Benedictis et al. 2014, 2016; Wang et al. 2016; Sarubbo et al. 2016; Hau et al. 2017).

More recently, in vivo neuroimaging methods have been developed for exploring the properties of white matter tracts and represent a rapidly expanding approach to studying the brain. Diffusion weighted imaging (dMRI) (Basser and Jones 2002; Hagmann et al. 2006; Mori and Zhang 2006)) and tractography (Mori et al. 1999; Basser et al. 2000; Lazar et al. 2003; Behrens et al. 2003; Descoteaux et al. 2009; Jbabdi and Johansen-Berg 2011; Wasserthal et al. 2018), for example, have been combined to study both the microstructural (tissue) and macrostructural (anatomical) properties of the human white matter in vivo (Jbabdi et al. 2015; Wandell 2016; Rokem et al. 2017; Yeatman et al. 2018). Indeed, dMRI and tractography have been used to identify the gross anatomical features of major, canonical tracts, such as the SLF (Makris et al. 2004; de Schotten et al. 2011), the Arc (Catani et al. 2002, 2005; Catani and Thiebaut de Schotten 2008), the IFOF (Catani et al. 2002; Wakana et al. 2007; Catani and Thiebaut de Schotten 2008; Sarubbo et al. 2013) or the ILF (Mori et al. 2002; Catani et al. 2002). These modern investigations reproduce the earlier findings of conventional post mortem dissection methods (Mori et al. 2005; Schmahmann et al. 2007; Catani and de Schotten 2012; De Benedictis et al. 2014; Mai et al. 2015; Wang et al. 2016; Sarubbo et al. 2016). We note that some discrepancies still exist between the description of white matter across primate species, in that some descriptions of the ILF, Arcuate, IFOF and MdLF might still be contentious (Schmahmann et al. 2007; Petrides and Pandya 2009; Forkel et al. 2014; Takemura et al. 2017). One issue that may account for these discrepancies is the current lack of established methods for unequivocally matching the very large number of connections that can be reported using tracer methods in the non-human primate brain (e.g., (Mori et al. 2005; Schmahmann and Pandya 2006; Schmahmann et al. 2007; Catani and de Schotten 2012)) with white matter tracts reported using dMRI, Klinger's dissection methods or polarized light imaging in the human brain (Dohmen et al. 2015; Decramer et al. 2018; Axer et al. 2011; De Benedictis et al. 2014)

Notably, the opportunity offered by in vivo methods in the human brain goes beyond anatomical confirmation. In vivo measurements also allow for correlations of the micro- and macro-structural properties of the white matter tracts with measures of human cognition and perception, in both healthy and disease states (Fields 2008a; Scholz et al. 2009; Yeatman et al. 2011; Hart 2015; Allen et al. 2015; Libero et al. 2016). Indeed, over time, the associative tracts have been found to be involved with communicating specific functional capacities. For example, the Arc has been linked to language (Catani and Mesulam 2008; Dick and Tremblay 2012; Hau et al. 2017), while the SLF has been linked to spatial planning and attention (Catani et al. 2005; Ptak and Schnider 2010; de Schotten et al. 2011; Friederici 2011; Bartolomeo et al. 2012; Lunven et al. 2015). Because understanding the human brain, perception and cognition are primary goals of neuroscience, 
advancing norms for identifying major tracts reliably in the living brain is a significant goal (Wandell 2016; Pestilli 2018).

Convergent evidence from ex vivo and in vivo methods has brought much attention to the canonical tracts, as demonstrated by the number of citations referring to the four example tracts we focus on here in this study (Figure 1b).(Yeatman et al. 2014; Wandell 2016; Pestilli 2018)
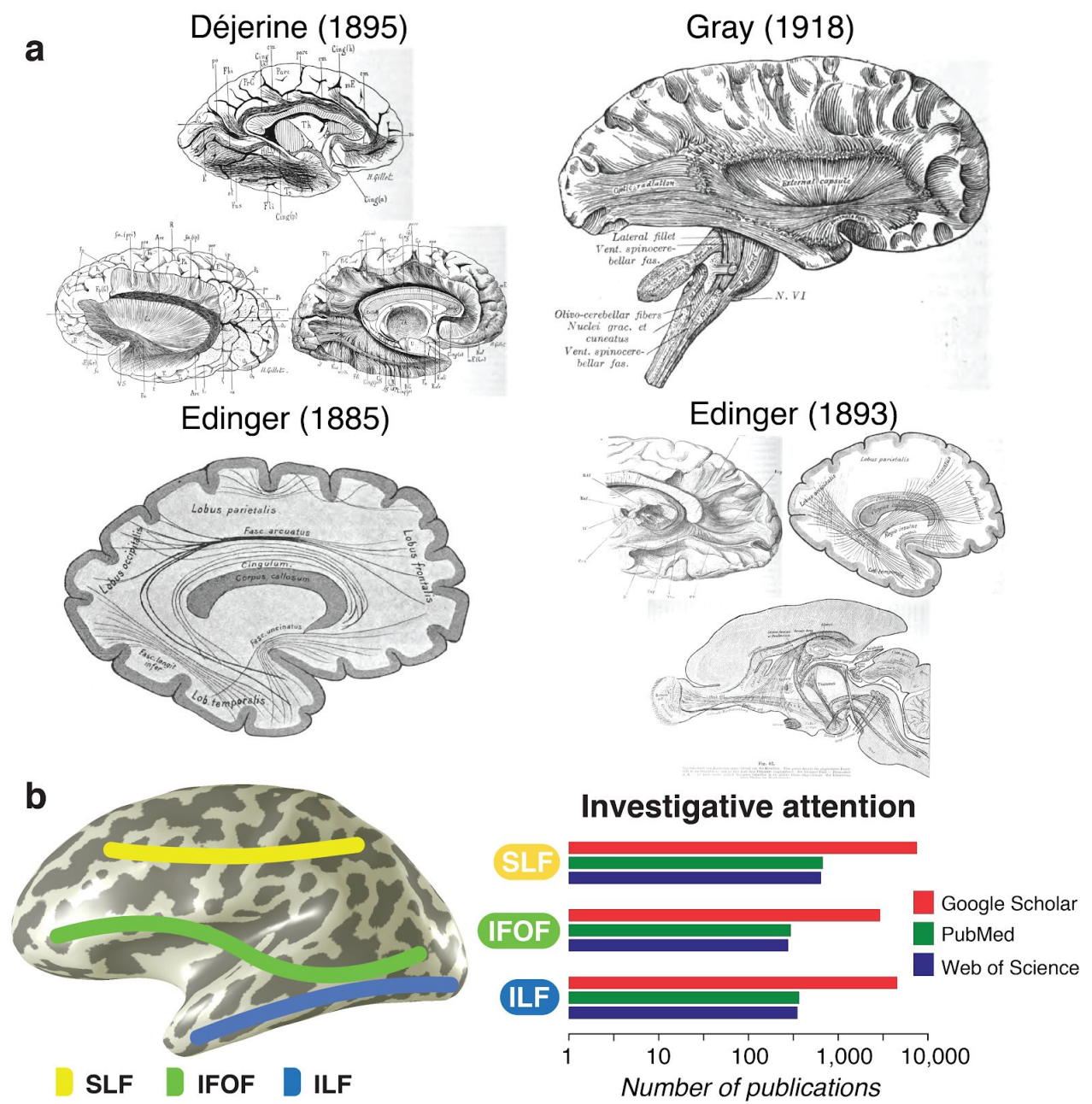

Fig. 1 Canonical associative tracts. A. Historical drawings of canonical tracts. Illustrations of major white matter tracts which focus on on anterior-posterior oriented tracts by Déjerine(Dejerine and Dejerine-Klumpke 1895), Gray (Gray 1918) and Edinger (Edinger 1885, 1893). B. Schematic depiction of canonical tracts and investigative attention. Right-hand graphs shows the number of articles mentioning each tract as returned from Google Scholar, PubMed, and Web of Science search results for the white matter tracts of interest (citation number in the ordinate, and white matter tract names in the abscissa). Search results were generated using the format: "[white matter tract name]" brain. For example: "Arcuate Fasciculus" brain. Searches conducted in April 2017

In addition to associating white matter characteristics with human cognition and disease, in vivo work has recently proven to be useful in examining a series of underreported tracts, thereby demonstrating that these methods can expand the boundaries of what is understood about human white matter, even beyond the established canon (Yeatman et al. 2014; Gomez et al. 2015; Leong et al. 2016; Wandell 2016; Rokem et al. 2017). This is despite evidence that in vivo methods may be limited in their capabilities (Thomas et al. 2014; Reveley et al. 2015). Regardless, several vertically-oriented associative white matter tracts have been reported recently using in vivo methods, examples of interest for the current article include the vertical occipital fasciculus (VOF; (Yeatman et al. 2014; Takemura et al. 2016b; Wu et al. 2016; Weiner et al. 2016; Wandell 2016; 
Lee Masson et al. 2017)), the posterior arcuate (pArc; (Catani et al. 2005; Weiner et al. 2016)), the Temporal-Parietal connection to the Superior Parietal Lobule (TP-SPL; (Kamali et al. 2014b; Wu et al. 2016)) and the middle longitudinal fasciculus (MdLF, (Makris et al. 2009; Menjot de Champfleur et al. 2013; Maldonado et al. 2013; Wang et al. 2013; Kamali et al. 2014b)). These tracts connect the dorsal and ventral cortical streams (Ungerleider and Haxby 1994; Milner and Goodale 2008), with profound implications for the functional architecture of the human connectome (Takemura et al. 2016b; Wu et al. 2016; Weiner et al. 2016; Lee Masson et al. 2017) and seem to be conserved in primate species (Takemura et al. 2017, 2018). Some of these non-canonical tracts that have received renewed attention via in vivo methods have been previously described using post mortem methods (Figure 2a (Obersteiner 1889; Dejerine and Dejerine-Klumpke 1895; Curran 1909; Gray 1918)). Others have only been reported using in vivo methods (i.e. the TP-SPL and MdLF-Ang).

Supplementary Figure 1 reports various previous characterizations of these tracts of interest and the methods used to identify them. The figure shows that all non-canonical tracts discussed here have been previously reported by multiple groups using imaging methods, and that two out four of these tracts have further been characterized via post-mortem dissection methods. We note that previous investigations did not consider the canonical, and non-canonical tracts in relation to one another in great detail. This is a major contribution of the present article. Broadly speaking, these vertical, non-canonical associative tracts have thus far been underrepresented in the investigations of white matter (Figure $\mathbf{2 b}$ ). We note the striking difference in literary attention between the extensive discussion of the Arc, SLF, IFOF and ILF (Figure 1b) as compared to the relative dearth of articles discussing the VOF, pArc, TP-SPL and MdLF (Figure 2b). 
a

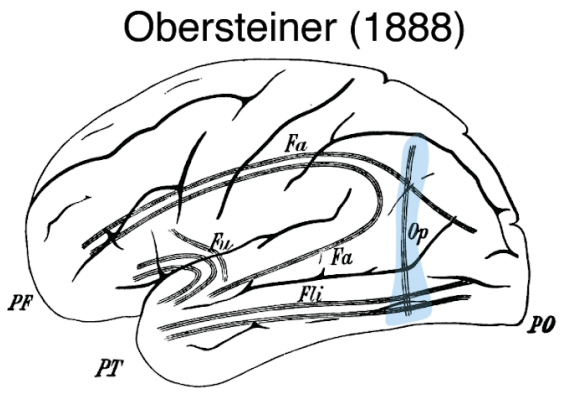

Déjerine (1895)

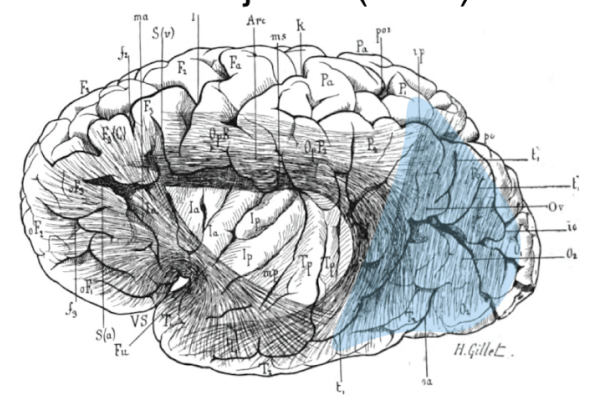

Curran (1909)

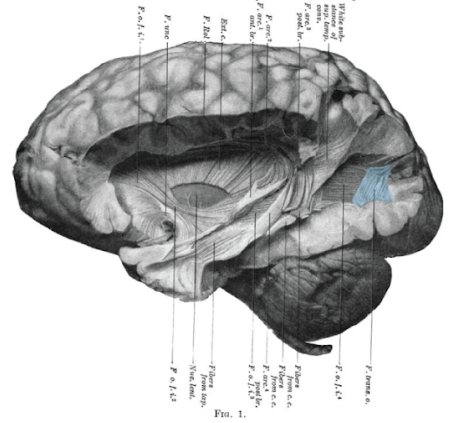

Gray (1918)

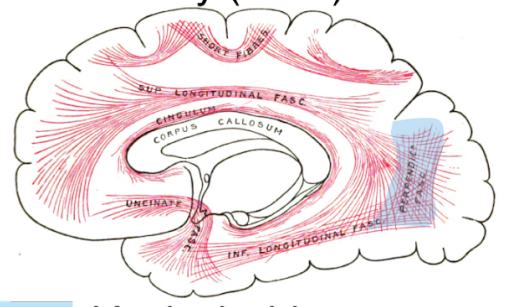

Vertical white matter

b

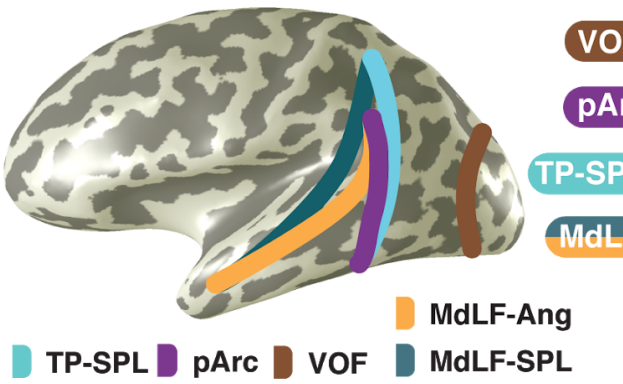

Investigative attention

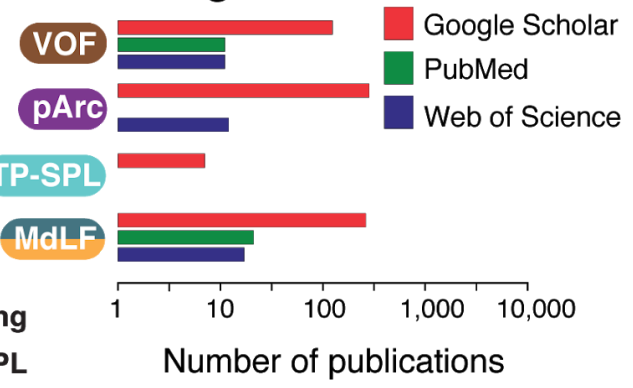

Fig. 2 Non canonical associative tracts. a. Historical drawings of canonical tracts with partial depictions of non-canonical tracts. Illustrations of white matter anatomy from Obersteiner (Obersteiner 1889, 1890), Curran (Curran 1909), and Déjerine (Dejerine and Dejerine-Klumpke 1895) and Gray (Gray 1918) featuring vertically oriented tracts in the posterior of the brain. B. Schematic depiction of canonical tracts and investigative attention. Right-hand graph shows the number of articles mentioning each tract as returned from Google Scholar, PubMed, and Web of Science search results for the white matter tracts of interest (citation number in the ordinate, and white matter tract names in the abscissa). Search results were generated using the format: "[white matter tract name]" brain. For example: "Arcuate Fasciculus" brain. Searches were conducted on April 252017

The primary aim of this article is to characterize in detail and compare canonical and non-canonical tracts. Despite growing evidence for multiple tracts connecting the human dorsal and ventral posterior cortices, a characterization of the trajectory and cortical terminations of the canonical and non-canonical tracts together has yet to be provided. With this work, we present an extensive anatomical and quantitative characterization of the properties of the non-canonical white matter tracts, including the middle longitudinal fasciculus (MdLF) and its two subcomponents (MdLF-Ang and -SPL), posterior arcuate (pArc), vertical occipital fasciculus (VOF), and temporo-parietal connection to the superior parietal lobule (TP-SPL). When relevant, we compare cortical terminations of these tracts to the aforementioned four canonical tracts, the superior longitudinal fasciculus (SLF), the arcuate fasciculus (Arc), the inferior longitudinal fasciculus (ILF), and inferior fronto-occipital fasciculus (IFOF). The secondary aim of this article is to also provide software for the automated segmentation of the VOF, MdLF, pArc and TP-SPL. The software is presented as both a stand-alone suite of scripts (https://github.com/brainlife/Vertical Tracts), a fully automated pipeline to perform the white matter 
segmentations presented here on new, user uploaded data sets on the cloud computing platform

https://brainlife.io (see Table 1). Data underlying the maps presented here can be found on the brainlife.io data publication (Bullock et al. 2019).

\section{RESULTS}

This study reports three major results to provide a reference for future research. First, we report the anatomical properties of the eight white matter tracts, starting with the four canonical anterior-posterior connective tracts, followed by the four less studied dorso-ventral connective tracts (Figures 3-4, Supplementary figure 2). We then report quantitative properties of the tracts (volume, length and statistical evidence; Figure 5), to provide a guide for other researchers studying these tracts. Finally, we examine the cortical terminations of the tracts, comparing the location and extent of these terminations for canonical and non-canonical tracts (Figures 6-16, Supplementary figure 4).

\section{Anatomical characterization of canonical and non-canonical tracts.}

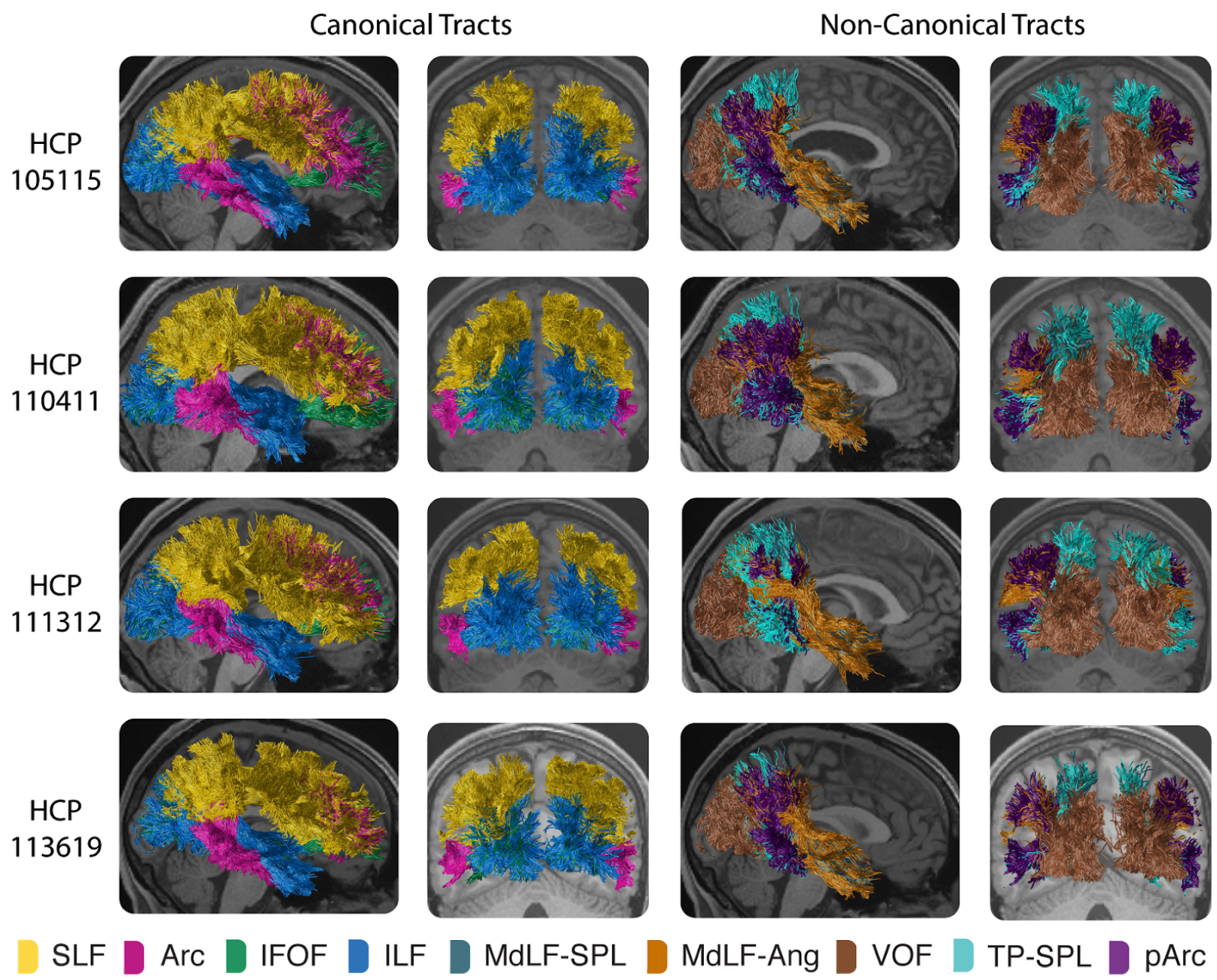

Fig. 3 Anatomy of major canonical and non-canonical white matter tracts. Columns 1 and 2: Sagittal and coronal view of canonical, horizontal, white matter tracts. Depicts a sagittal cross section of a human brain with the superior longitudinal fasciculus (SLF) in yellow, arcuate fasciculus (Arc) in pink, inferior fronto-occipital fasciculus (IFOF) in green, and inferior longitudinal fasciculus (ILF) in blue. Columns 3 and 4: Sagittal and coronal view of non-canonical, dorso-ventral connecting white matter tracts. A sagittal and coronal cross section with the Temporo-Parietal connection to the superior 
parietal lobule (TP-SPL) in cyan, posterior arcuate (pArc) in purple, middle longitudinal fasciculus - superior angular gyrus component (MdLF-Ang) in orange, middle longitudinal fasciculus - superior parietal lobule component (MdLF-SPL; note that this component is partially obscured by the MdLF-Ang), and the vertical occipital fasciculus (VOF) in copper. Coronal cross section positioned at $-47 \mathrm{~mm}$ from the posterior commissure, coronal plane). See Supplementary Figure 2 for subjects from STN dataset.

In order to examine the anatomy of the eight tracts of interest (TOI), we first had to generate candidate connectomes (defined here as the full set of streamlines covering the total white matter volume (Pestilli et al. 2014; Goldstone et al. 2015; Takemura et al. 2016a; Caiafa and Pestilli 2017) from which the TOls could be extracted. To this end, we utilized dMRI data of eight individual brains from both the STN and HCP 3T data sets (see Methods). We used probabilistic tracking to generate ten (repeated measures) whole brain connectomes using multiple tracking methods (constrained-spherical deconvolution based tractography with $L_{\max }=2-10$ ) (Tournier et al. 2012; Takemura et al. 2016a). From each of these whole brain connectomes, 100,000 streamlines were selected and incorporated into a multi-parameter, whole brain tractography ensemble (Tournier et al. 2012; Takemura et al. 2016a). These ensembles were, in turn, validated using the LiFE methodology. Streamlines that did not contribute to the prediction of the measured diffusion signal in individual brains were, therefore, not included in subsequent analyses as there was no evidence for their existence (Pestilli et al. 2014; Takemura et al. 2016a, 2017; Caiafa and Pestilli 2017). Tracts were segmented using a region of interest based method (see also Methods). Anatomical segmentations of the TOls in four example subjects are reported in Figure 3 and Supplementary Figure 2. Plots show overall tract anatomy and degree of variability across subjects comparable to previous studies (Pestilli et al. 2014; Takemura et al. 2016a; Caiafa and Pestilli 2017).

We begin by looking at the anatomical features of the canonical tracts (SLF, ILF, IFOF and Arc). The left-hand column of Figure 3 presents sagittal views of these four predominantly horizontal tracts. These canonical tracts have been widely described in other works, with the SLF constrained to the superior-medial white matter of the frontal and parietal lobes, the ILF to the temporal and occipital, the Arc connecting temporal and frontal lobes and the IFOF spanning the occipital, temporal and frontal white matter (Figure 3).

Consistent with previous reports (de Schotten et al. 2011; Catani and de Schotten 2012), we show canonical tracts extending posteriorly into the parietal, temporal and occipital lobes, and anteriorly either toward prefrontal cortex (SLF, Arc (Makris et al. 2004; Martino et al. 2013b); IFOF (Martino et al. 2010b, a)) or into the anterior temporal lobe (ILF (Davis 1921)). We next look at the anatomical features of the non-canonical tracts (MdLF, pArc, TP-SPL and VOF). The third and fourth column of Figure 3 present sagittal and coronal views of the four tracts. These tracts are posterior to the corpus callosum (with the exception of the temporal portion of the MdLF). All of these tracts occupy the posterior ventral and dorsal white matter volume, and as a consequence, they are typically shorter than the canonical tracts (with the exception of the MdLF; Figure 3 columns 3 and 4). The VOF is by definition restricted exclusively to the occipital white matter (Figure 3 column 3, copper; (Yeatman et al. 2014; Takemura et al. 2016b; Rokem et al. 2017)). A more in depth look at the morphologies of the TP-SPL, pArc and MdLF will be provided later (see Anatomy and cortical terminations of individual non-canonical tracts). 


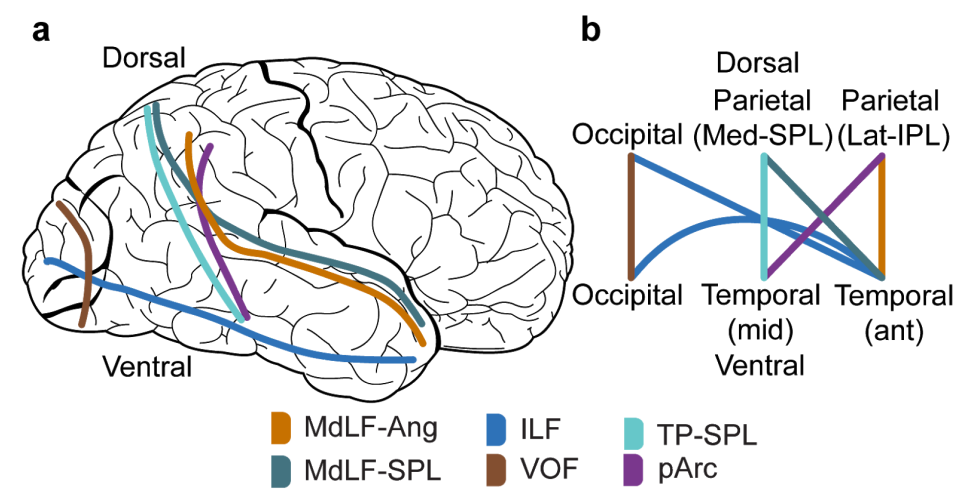

Fig. 4 Schematic summary of several anatomical projections in the occipital, temporal and parietal lobes. a. Anatomical connectivity information overlaid on schematic brain anatomy. The shape of the colored lines represents the rough anatomical path of each white matter tract. Color identifies different tracts. b. A schematic of the connectivity information as a limited graph. Nodes correspond to the specified cortical areas while edges correspond to the tracts themselves (as depicted by color). See also (Goryainov et al. 2017)

Hereafter we provide an extensive characterization of the canonical and non-canonical tracts. Before doing that, we provide a summary of the features of five TOls (including a sub-segmentation of the MdLF) that are less well characterized (Figure $\mathbf{2 b}$ ), which project within the occipital, temporal and parietal lobes (Figure 4). The figure can be used by the readers as reference as the fine anatomical organization is presented below. The schematic in Figure 4 depicts a summary of the general path-morphology and connectivity pattern of the tracts of interest terminating the posterior cortex (parietal and occipital lobes). We show a pattern of cortical terminations. First, two streams connect the middle temporal lobe with superior and lateral parietal (TP-SPL and pArc, respectively; Figure 4a cyan and purple). Second, two streams connect the anterior temporal lobe with the superior and lateral parietal lobule (MdLF-SPL and - Ang, respectively Figure 4a jade and orange). In addition, the occipital lobe connects to the anterior temporal lobe via the ILF (Figure 4a blue), while the VOF connects the dorsal and ventral streams within the occipital lobe (Figure 4a copper). The interest in this analysis is in highlighting the pattern of connectivity of the tracts connecting within the occipital, temporal and parietal lobes, which corresponds to the early components of the dorsal and ventral visual-associative pathways. The schematic in Figure 4a summarizes the findings with an additional level of abstraction. This schematic representation highlight the crossing pattern of double-stream communication between the parietal and temporal lobes and the relatively more isolated connectivity in the occipital lobe. The results indicate strong convergence from parietal cortex within two regions of the temporal lobe (anterior and medial). We provide a deep characterization of the major features of the TOls measured using diffusion imaging and tractography.

\section{Quantitative characterization of canonical and non-canonical tracts}

We provide a series of quantitative measurements that can be established using tractography: tract length, volume and "strength of evidence" - the latter is hereafter measured and referred to as the earth mover distance or EMD; (Pestilli et al. 2014). Examination of these features supports several observations. 


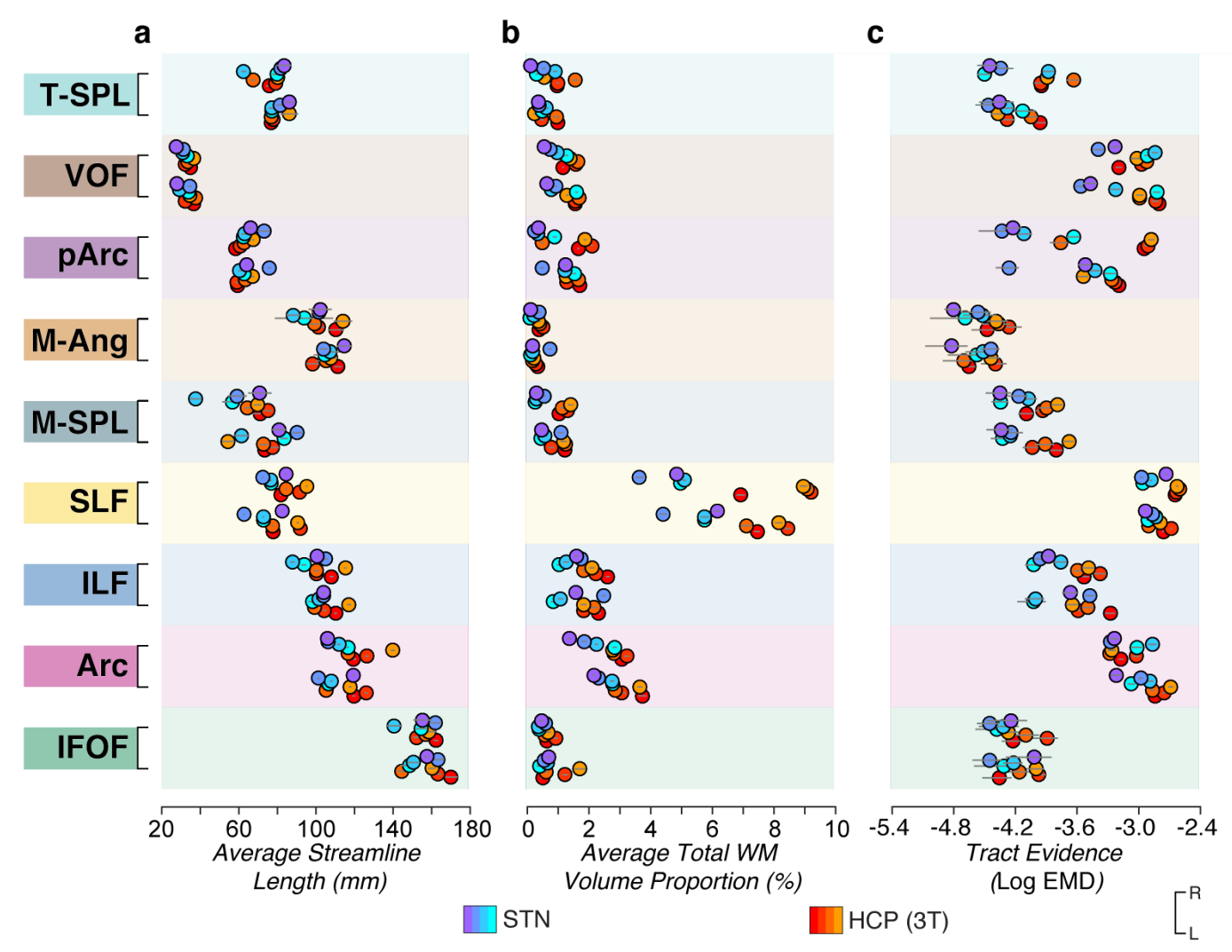

Fig. 5 Quantitative characterization of canonical and non-canonical white matter tracts. a. Tract length in $m m$ for each subject and different data sets averaged across repeated segmentations to establish reliability. b. Percentage white matter volume occupied by tract relative to total white matter volume. c. Statistical evidence for each computed using the Earth Mover's Distance (EMD). All data plotted for seven subjects, 3 (in cool colors) from the STN data set and 4 (in warm colors) from the HCP 3T data set. Values for the left and right hemispheres tacts are plotted one above each other as indicated by the ticks in the ordinate axes. Error bars are computed, per subject and tract, by calculating the standard deviation of each measure obtained across ten repeated measures connectomes generated using identical parameter settings.

First, we note that the non-canonical tracts are shorter than the canonical ones (with the exception of the MdLF; Figure 5a). The proportion of total white matter volume (PTWM; ratio between TOI volume and whole white matter volume) reveals that the SLF is larger than any of the other tracts, occupying a PTWM of nearly twice that of the other tracts (Figure 5b). This may be attributable to the methods used for segmentation, which, in our case does not distinguish between the SLF's subcomponents (de Schotten et al. 2011).

Additionally, we observe that the estimates of the PTWM for tracts from STN subjects occupy less volume than their counterparts in HCP subjects - compare warm and cool colors (Figure 5b). This is likely due to lower data SNR (HCP 32.6704 \pm 11.3595 and STN 20.2100 \pm 10.2686 ) and the related reduction in the number streamlines after the LiFE algorithm (Caiafa and Pestilli 2017) is applied. See below for more details.

We utilized the LiFE algorithm implemented using the ENCODE framework (Caiafa and Pestilli 2017) to evaluate TOIs and exclude streamlines which were not supported by the data. The ENCODE method was also used to implement "virtual lesions" for individual tracts and to measure the statistical strength of evidence associated with each tract (Pestilli et al. 2014). Briefly, the virtual lesion method removes the TOI from a full-brain connectome and measures the increase in error in predicting the dMRI signal within the volume of the the TOI. The error of the two connectome models in all voxels touched by the TOI is compared between a model with and without the TOI. The difference in these two distributions of error is then compared by using the the Earth Mover's Distance (EMD; (Rubner, Y., Tomasi, C. \& Guibas, L.J. 2000; Pestilli et al. 2014)). 
Importantly, all EMD values are clearly above zero, indicating that all the TOls nontrivially contribute to prediction of the measured dMRI signal (Figure 5c). Generally the EMD values cover a wide range of values (hence the $\log _{10}$ scaling in Figure 5c), consistent with previous reports (Pestilli et al. 2014; Caiafa and Pestilli 2017). The strength of evidence measure based on EMD depends on data quality and tract size (Figure 5, compare warm and cool colors). Specifically, tracts identified on higher signal-to-noise data (HCP3T dataset) show higher EMD values than those identified on lower SNR data set. This is a replication of previous results (Pestilli et al. 2014).

As was the case with the length and volume proportion, several trends can be observed relative to our measure of evidence. A consideration of tract volume (Figure $5 \mathbf{b}$ ) alongside tract evidence (Figure 5c) hints that tract evidence is related to volume proportion (Pearson correlation coefficient $r=0.83$ across all tracts and subjects). One of the non-canonical tracts, the VOF, exhibits evidence comparable to that of the Arc and SLF (roughly 0.001 versus 0.0012 respectively). This is beside the fact that the VOF is much smaller volumetrically than either the Arc and SLF (about 1\% verses about 3\% and 6\% of the total WM volume, respectively; Figure $5 b)$.

The reproducibility and variability in quantitative properties for all tracts of interest were also topics of interest for this study. To this end, we exploited the ten repeated measures of tracking and statistical evaluation generated for each subject (see Methods: Repeated Measure Tract Generation). The high degree of consistency, as illustrated by the narrow grey bars in Figure 5, columns 1 and 2, indicates that the automated segmentation methods that we utilized reliably identified tracts with highly consistent features across multiple reconstructions. Errors in segmentation method would incorporate streamlines from extraneous tracts and thereby increase variability in both count and average length, however minimal variability in tract volume, length, or statistical evidence was noted (Figure 5a, b and c, note grey error bars). In sum, these results allow us to consider variability between and within subjects and compare these measures across tracts. In order to establish norms, however, it will be useful to look at the specific quantitative traits associated with these tracts - this is discussed with the use of Supplementary Tables 1 and 2.

Examination of these quantitative features supports several observations. First, we note that the non-canonical tracts are shorter than the canonical ones (with the exception of the MdLF; Figure 5a). Second, tract volume, as a proportion of total white matter volume, is smaller for the non-canonical tract (Figure 5b). Third, strength of evidence is comparable across canonical and noncanonical tracts, and the EMD measures correlate with tract volume (Figure 5c). These quantitative properties are taken to be characteristic of their respective tracts and to aid comparisons across canonical and non-canonical tracts.

\section{Anatomy and cortical terminations of individual non-canonical tracts}

Below we describe the anatomical path and cortical terminations of noncanonical tracts individually. We focus on the spatial occupancy and extent of cortical projection overlap between canonical and non-canonical tacts in the later sections. We avoid discussing the VOF because its cortical terminations has recently been discussed in great detail elsewhere (Takemura et al. 2016b). Supplementary Figure 3 provides, as reference for the reader, the major anatomical landmarks on a labeled cortex (Mai et al. 2015). Multiple methods and parameter sets can be used for estimating the cortical terminations of white matter tracts using tractography. Choices in such methods can affect the final results and estimates (e.g., (Goldstone et al. 2015; Takemura et al. 2016b, 2017)). 

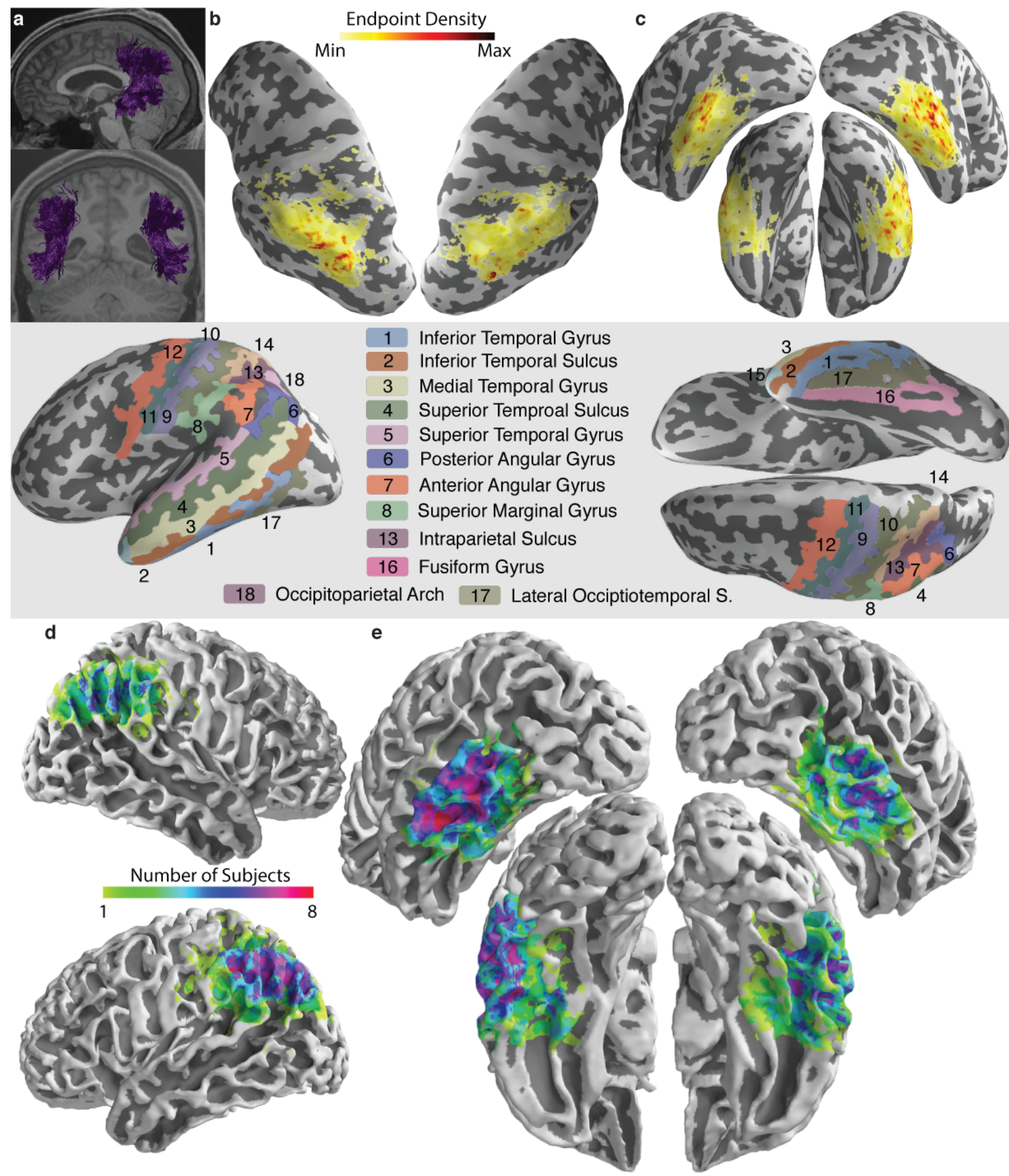

Fig. 6 Posterior arcuate cortical termination pattern. a. Posterior arcuate anatomy. Anatomy of the tracts of interest is plotted over representative sagittal and coronal brain slices for a single subject (HCP 105115).. b. Superior cortical endpoint density mapping, pArc. c. Inferior cortical endpoint density mapping, pArc. Density projection plotted on both cortical hemispheres summed across all subjects. Darker coloring of the heat map corresponds to higher densities of nearby endpoints. Shaded inset shows pertinent cortical areas including regions 1 and 3 and $6-8$, see also

Supplementary Figure 3. $d$ and e. Superior and inferior consistency maps across subjects of the pArc. Maps show binarized endpoint density counts across 8 subjects. 
Cortical termination calculations are further complicated by the existence of cortical white matter that can limit the accuracy of tractography at the interface between white matter and gray matter (Reveley et al. 2015). For these reasons, our data visualization approach is relatively generous in depicting the cortical terminations of each tract. In short, we first applied a gaussian smoothing kernel with a $7 \mathrm{~mm}$ radius to the tract endpoints and then used PySurfer (see Methods; https://pysurfer.github.io) to plot the resulting data onto the cortical surface. Data were minimally thresholded - only density values less than $0.5 \%$ of the maximal post smoothing density were left out. This is to provide liberally estimated cortical areas of putative impact of each tract. It is likely that future improvements in tractography methods will result in more conservative estimates of these endpoint mappings. At this point however, we are interested in clarifying potential interactions and overlap between tract termination zones.

The posterior arcuate (pArc; Figure 6a) is a vertically oriented tract connecting the inferior parietal with the middle and inferior temporal lobes, the pArc forms a subcomponent of the arcuate fasciculus (Catani et al. 2005; Catani and Mesulam 2008; Kamali et al. 2014a, b; Weiner et al. 2016). Previous work has referred to this tract using a multitude of names including, the SLF-V (Wu et al. 2016), SLF TP-IPL (Kamali et al. 2014a, b), AFv/vAF (Makris et al. 2004; Panesar et al. 2019), vertical SLF (Martino et al. 2013a; Martino and De Lucas 2014), perisylvian SLF (Catani and Ffytche 2005; Martino et al. 2013b), posterior SLF (Martino et al. 2013b), indirect AF (Turken and Dronkers 2011), and temporo-parietal aslant (Panesar et al. 2019). We describe the pArc's dorsal terminations as occurring lateral to the intraparietal sulcus, in the inferior parietal lobule (IPL), and superior to the posterior portion of the lateral fissure (Figure 6b; see also regions 6, 7, and 8 in Supplementary Figure 3). The ventral terminations occur throughout the posterior and middle temporal lobe, specifically in the middle and inferior temporal gyrus (Figure 6c and 6e; see also Supplementary Figure 3 regions 1 and 3 ). These terminations are not apparent in the temporal pole. Importantly, minimal, if any, connectivity is noted in the fusiform gyrus ( see Supplementary Figures 6 and 8, Supplementary Figure 3 region 16).

The temporo-parietal connection (TP-SPL; Figure 7a; (Kamali et al. 2014a)) also referred to as the TP (Wu et al. 2016) is another non-canonical, dorso-ventrally oriented tract connecting the temporal and parietal lobes. In contrast to the more compact pArc, we find that the TP-SPL is better characterized by a sheet-like morphology, and connects the superior parietal lobule with the middle and inferior temporal lobe. The TP-SPL's superior terminations are located medial to the IPS, after which the tract adopts an oblique orientation as it descends from the parietal lobe to the ventro-lateral temporal lobe (Figure $\mathbf{7 b}$ ). In dorsal cortex, its terminations are bordered anteriorly by the postcentral gyrus (Supplementary Figure 3, area 9 ) and posteriorly by the dorsal IPS (Supplementary Figure 3, area 13), as well as by the occipitoparietal arch (Mai et al. 2015) (Supplementary Figure 3, area 18). The inferior terminations occupy the middle and posterior portions of the middle and inferior temporal gyri (Figure 7C; Supplementary Figure 3, areas 1 and 3). The TP-SPL does not extend to more anterior temporal regions, nor does it extend into ventral occipital regions. Finally, it exhibits several areas of high endpoint density within the fusiform gyrus (see Supplementary Figures 6 and 8, see Supplementary Figure 3, area 16; (Wu et al. 2016)). 

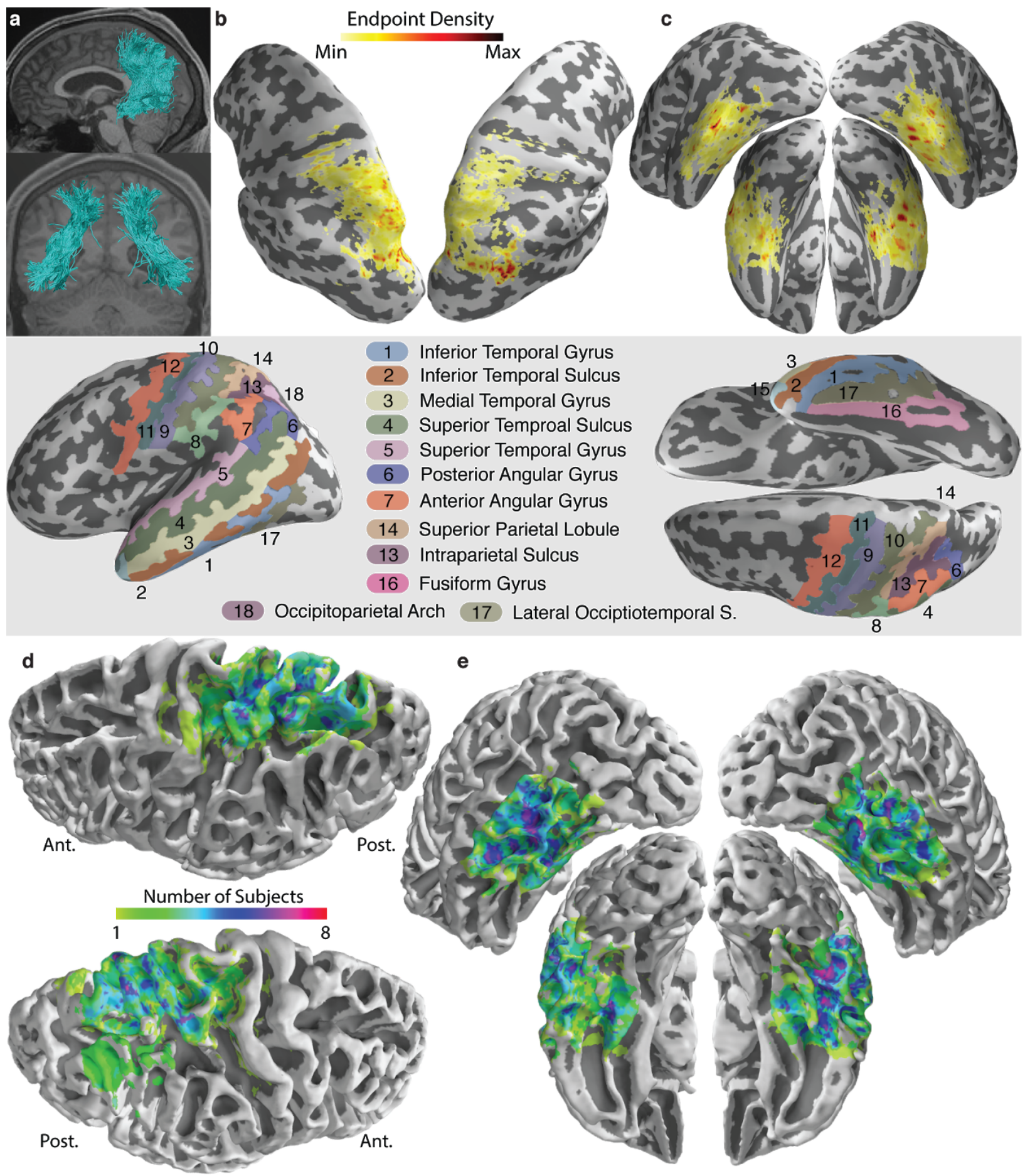

Fig. 7 Temporo-parietal connection cortical termination pattern. a. TP-SPL anatomy. Anatomy of the tract of interest is plotted over a representative sagittal and coronal brain slice for a single subject (HCP 105115). b. Superior cortical endpoint density mapping, TP-SPL. c. Inferior cortical endpoint density mapping TP-SPL. Density projection plotted on both cortical hemispheres summed across all subjects. Darker coloring of the heat map corresponds to higher densities of nearby endpoints. Shaded inset shows pertinent cortical areas including regions 1 and 3 and 6-8, see also Supplementary Figure 3. d and e. Superior and inferior consistency maps across subjects of the TP-SPL. Maps show binarized endpoint density counts across 8 subjects. 

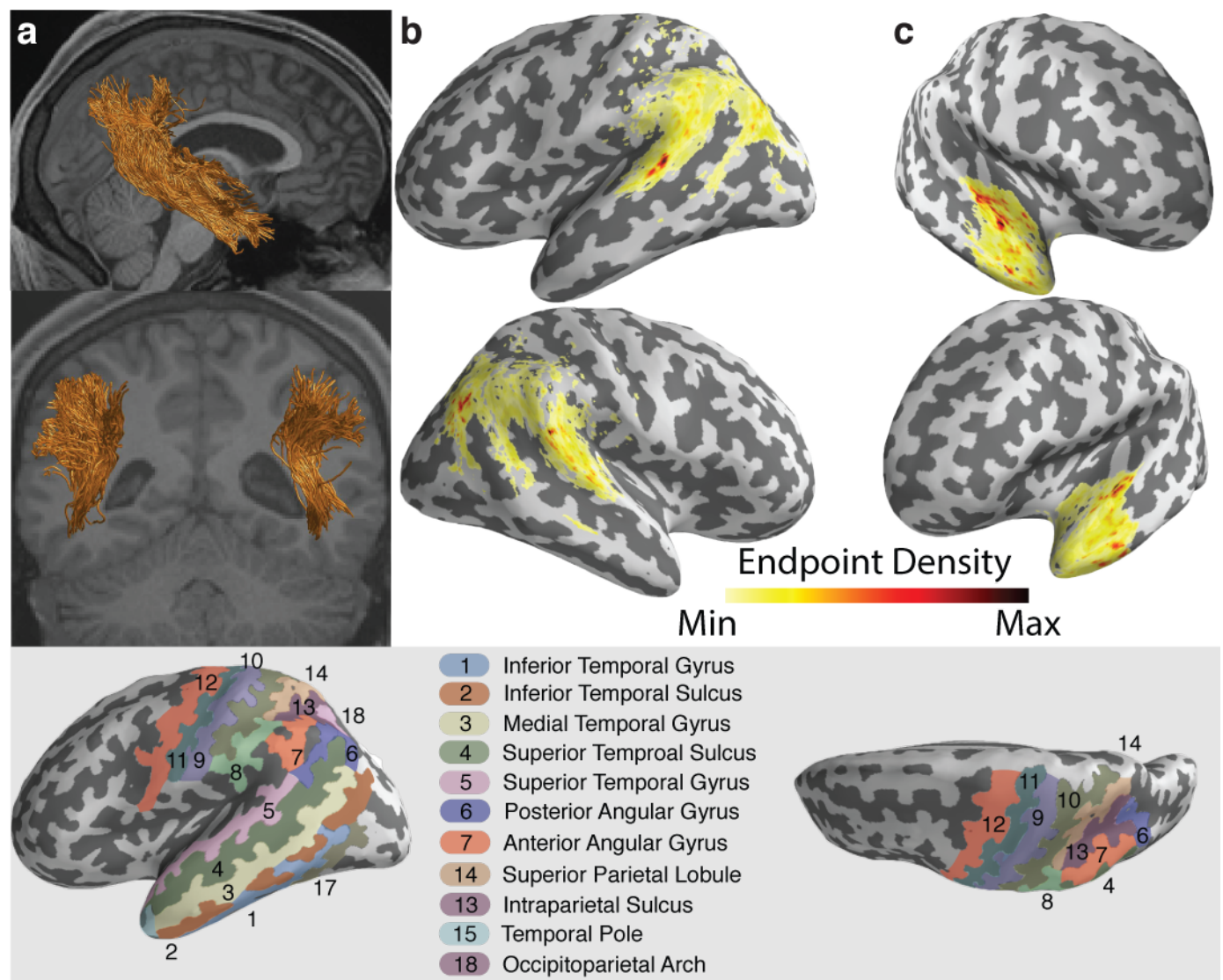

\section{Min}

1 Inferior Temporal Gyrus

(2) Inferior Temporal Sulcus

3 Medial Temporal Gyrus

(4) Superior Temproal Sulcus

5 Superior Temporal Gyrus

6 Posterior Angular Gyrus

7 Anterior Angular Gyrus

14 Superior Parietal Lobule

13 Intraparietal Sulcus

15 Temporal Pole

18 Occipitoparietal Arch
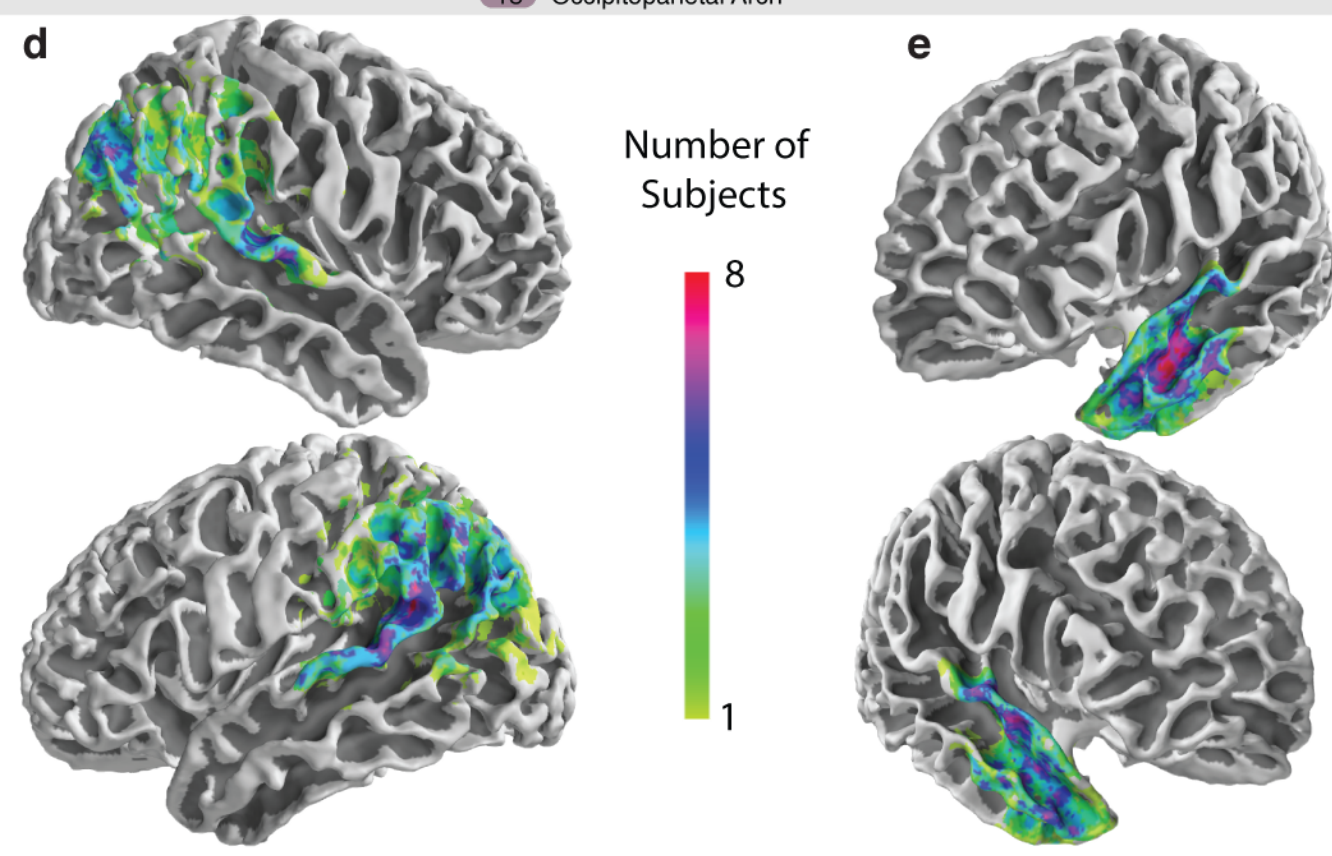

Fig. 8 MdLF-Ang cortical termination pattern. a. MdLF-Ang anatomy. Anatomy of the tract of interest is plotted over a representative sagittal and coronal brain slice for a single subject (HCP 105115). b. Superior and posterior cortical endpoint density mapping, MdLF-Ang. c. Inferior and anterior cortical endpoint density mapping, MdLF-Ang. Density projection plotted on both cortical hemispheres summed across all subjects. Darker coloring of the heat map corresponds to higher densities of nearby endpoints. Shaded inset shows pertinent cortical areas including regions 1 and 3 and 6-8, see also Supplementary Figure 3. d and e. Superior and inferior consistency maps across subjects of the MdLF-Ang. Maps show binarized endpoint density counts across 8 subjects. 
The angular gyrus component of the middle longitudinal fasciculus (MdLF-Ang or MdLF-IPL) is a somewhat more obliquely oriented tract, as compared to the PArc and TP-SPL, and connects the temporal and parietal lobes. Specifically, it connects the anterior temporal regions to the angular gyrus, inferior parietal lobule (IPL) and some regions of posterior superior temporal gyrus (Menjot de Champfleur et al. 2013; Makris et al. 2013a, 2017; Kamali et al. 2014a, b; Bajada et al. 2015). Its posterior morphology is quite similar to the pArc and is similarly characterized by its superior terminations being located lateral to the IPS (compare Figure 8a and Figure 6a). In the anterior temporal lobe, a high density of terminations is noted in the anterior superior temporal gyrus (see Supplementary Figure 5 and 8, Supplementary Figure 3, area 3).

The superior parietal lobule component of the middle longitudinal fasciculus (MdLF-SPL), like the MdLF-Ang, is an obliquely oriented tract which connects the temporal and parietal lobes. Unlike the MdLF-Ang, the MdLF-SPL's superior terminations are located medial to the IPS and are spread along the superior parietal lobule (Figure 9b; (Lawes et al. 2008; Martino et al. 2013a; Makris et al. 2013a, 2017; Wang et al. 2013; Kamali et al. 2014a, b). Just as the morphology of the posterior MdLF-Ang mirrors the pArc, the posterior morphology of the MdLF-SPL mirrors the TP-SPL, most notably in the sheet-like character it exhibits (Figure 9a). The posterior termination pattern, which extends along the entirety of the superior parietal lobule, exhibits an increased density near the posterior border, in the occipitoparietal arch (Figure $\mathbf{9 b}$ and Supplementary figure 5 and 8, Supplementary Figure 3, area 18). Like the MdLF-Ang, there exists increased termination density in the anterior superior temporal gyrus of the anterior termination regions of the MdLF-SPL (Figure 9c, Supplementary Figure 3, area 3).

\section{Comparisons between pairs of noncanonical tracts}

Dorsally separated tract terminations. The TP-SPL is found in close proximity to the pArc (Figure 9a; (Wu et al. 2016)). The two tracts exhibit extensive volumetric overlap in the temporal lobe, with both tracts exiting the middle (MTG; Supplementary Figure 3, area 3) and inferior (ITG; Supplementary Figure 4, area 1) gyri of the temporal lobe and curving dorsally as they move into the parietal lobe. Although the inferior terminations of the tracts look largely similar, there are two particular features worth noting. First, we see that the pArc's terminations extend superiorly to the overlap region, indicating that the pArc has more extensive connections with the superior aspects of the middle temporal gyrus. Conversely, the TP-SPL is noted to have terminations on the ventral surface of the temporal lobe which extend more medially than the pArc (Figure 8c). These TP-SPL connections appear to extend into the fusiform gyrus, while the pArc lacks any such corresponding terminations in the fusiform gyrus (Figure 8c, Supplementary Figure 3, area 16). 


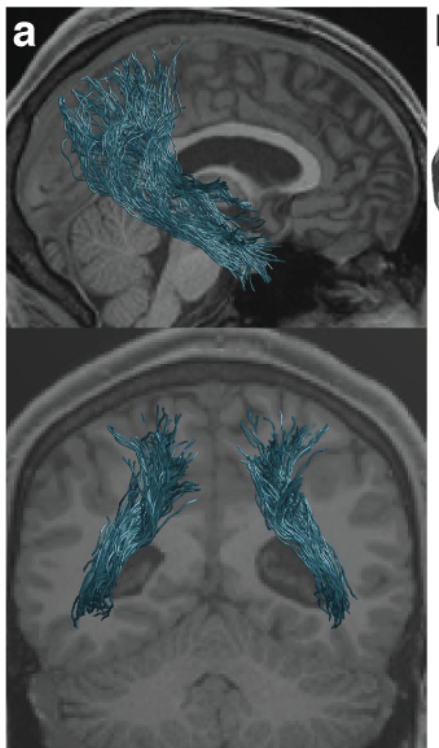

b

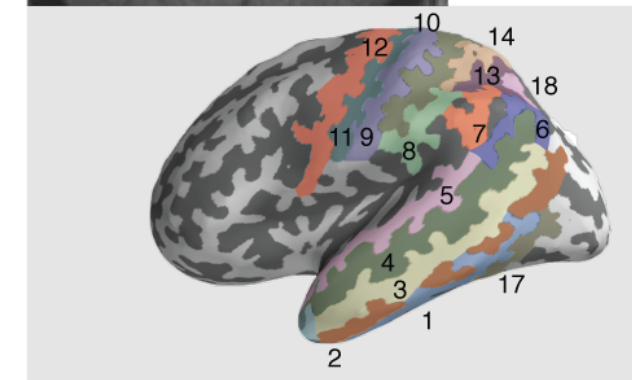

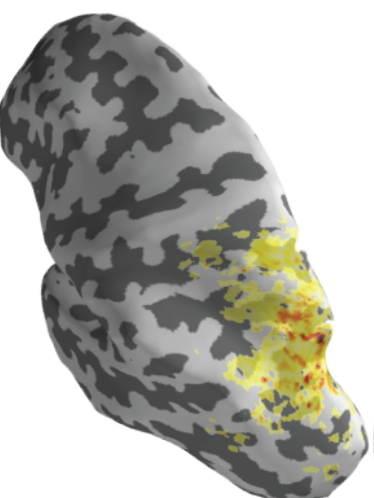

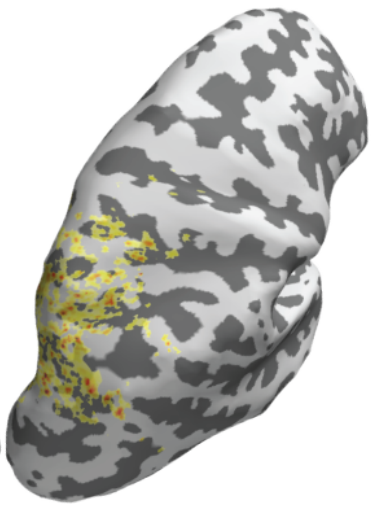

Endpoint Density

Min
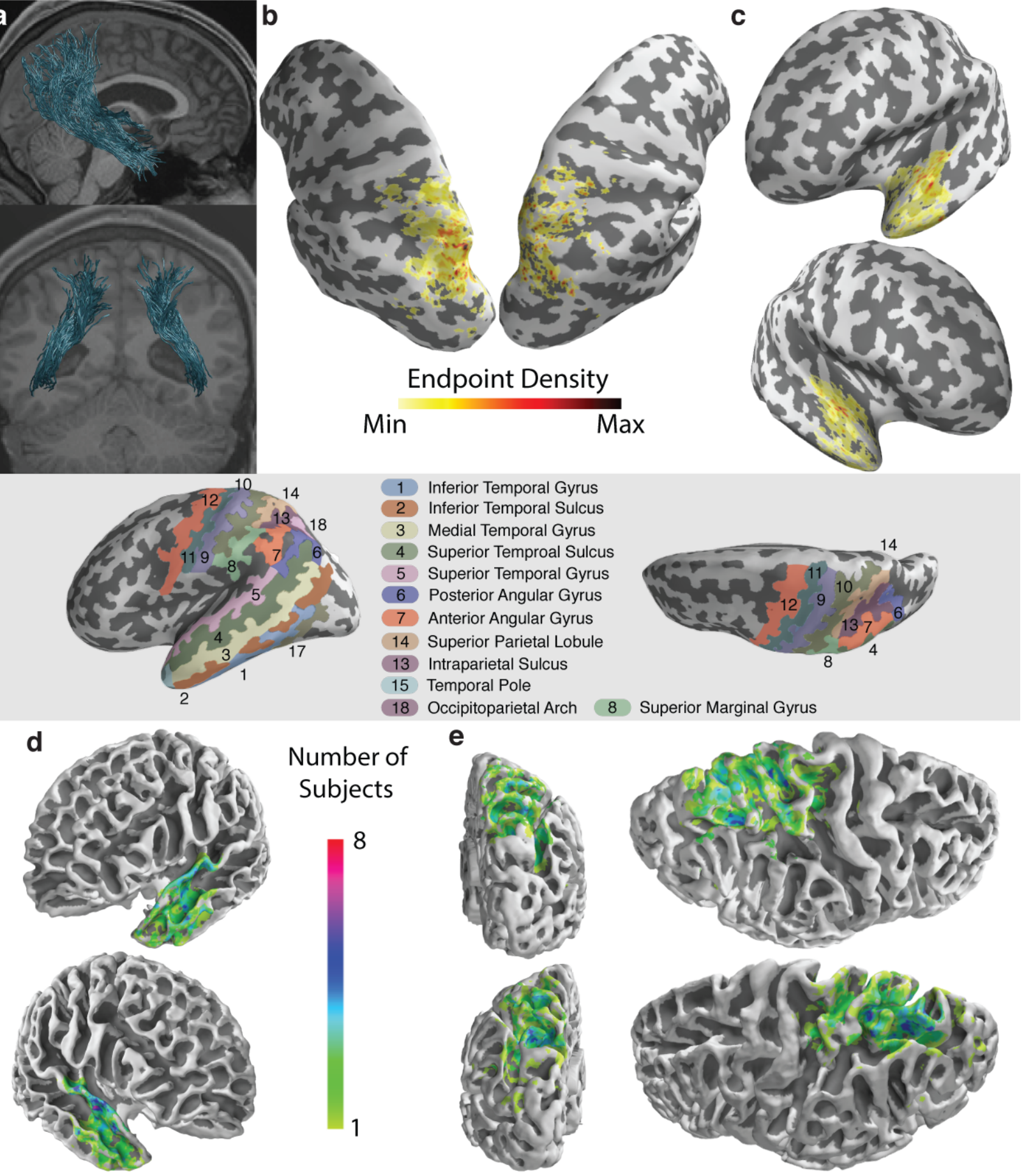

Fig. 9 MdLF-SPL cortical termination pattern. a. MdLF-SPL anatomy. Anatomy of the tract of interest is plotted over a representative sagittal and coronal brain slice for a single subject (HCP 105115). b. Superior and posterior cortical endpoint density mapping, MdLF-SPL. c. Inferior and anterior cortical endpoint density mapping, MdLF-SPL. Density projection plotted on both cortical hemispheres summed across all subjects. Darker coloring of the heat map corresponds to higher densities of nearby endpoints. Shaded inset shows pertinent cortical areas including regions 1-3 and 13-15, see also Supplementary Figure 3. $d$ and e. Superior and inferior consistency maps across subjects of the MdLF-SPL. Maps show binarized endpoint density counts across 8 subjects. 
The two tracts become more distinct from each other as they move dorsally (Figure 10a, coronal). The posterior arcuate adopts a vertical orientation before curving laterally back towards the IPL (Supplementary Figure 3, regions 6,7 , and 8) while the TP-SPL continues obliquely towards the superior parietal lobule (SPL) (Supplementary Figure 3, area 14). The IPS (Supplementary Figure 3, area 13) serves as a distinguishing feature for these tracts, with the TP-SPL's superior terminations being medial to the IPS and the pArc's being lateral to the IPS (Figures 10b and 10d). For both tracts the anterior portion of the superior terminations (Figure 10b) about the postcentral gyrus (Supplementary Figure 3, area 9) while the posterior terminations about the occipitoparietal arch (Supplementary Figure 4, area 18). The pArc is the denser of the two tracts (See Supplementary Table 1 and 2, count), and it also shows a larger termination area than the TP-SPL both superiorly and inferiorly (Figures 10b through e). It's worth noting that the apparent overlap observed in the right posterior parietal lobe and left anterior parietal lobe (Figure 10d) is likely due to the warp that was applied to align all subjects to the same atlas space (seem Methods). In the right posterior parietal lobe, this apparent overlap is probably attributable to variability in the mapping of the pArc (see Figure 6d, compare left and right hemispheres, note higher consistency in right hemisphere), while the apparent overlap in left hemisphere is attributable to a single subject's anatomical variation (see Figure 6d, left hemisphere). Inspection of individual subjects' mappings reveal no within-subject overlap of these two tracts (Supplementary Figures 6 and 8).

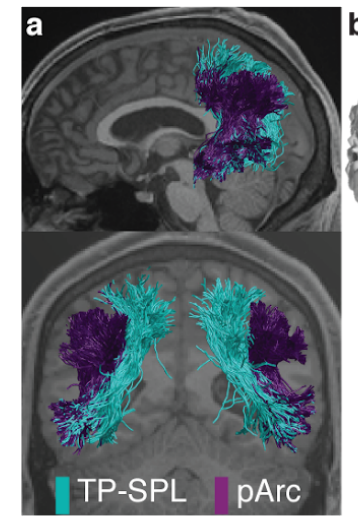

d
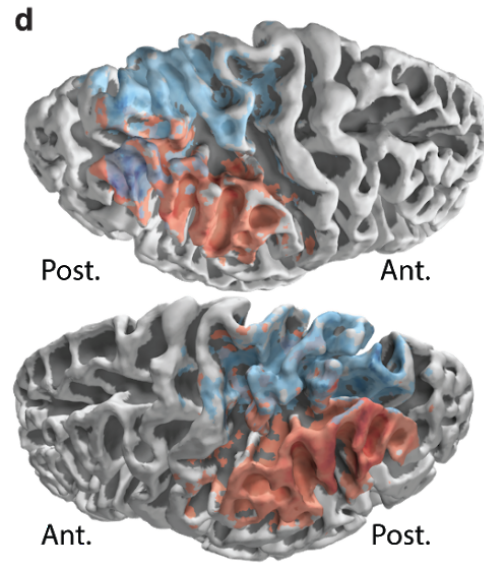
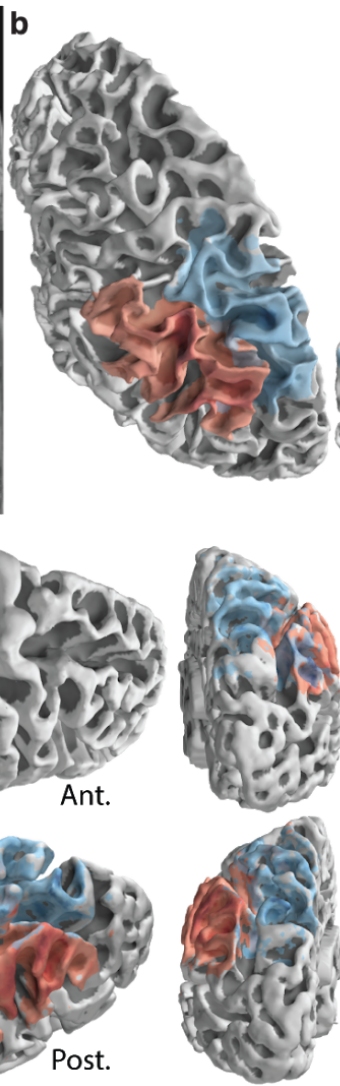
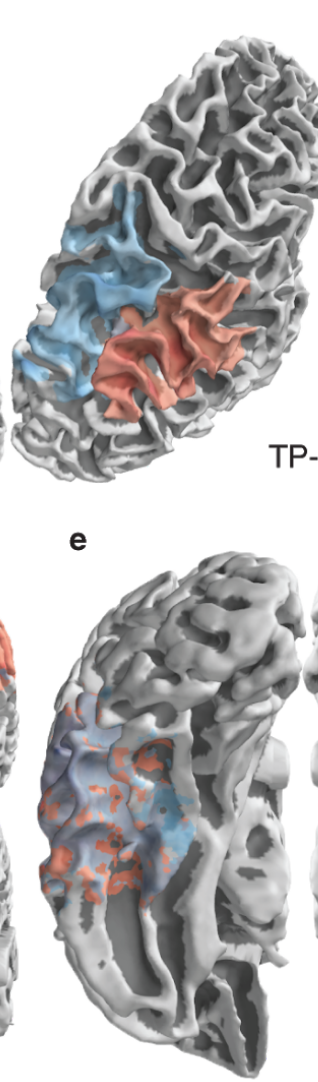
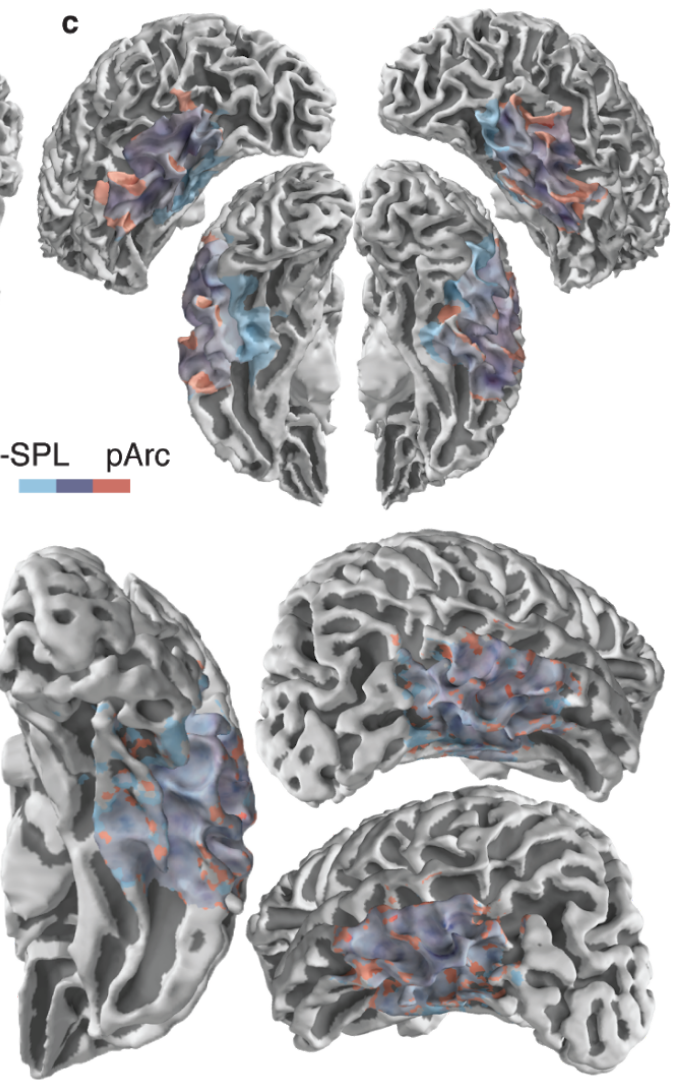

Fig. 10 Relation between the pArc and TP-SPL cortical termination patterns. a. Two tracts anatomy, pArc and TP-SPL. Anatomies of the two tracts of interest are plotted over a representative sagittal and coronal brain slice for a single subject (HCP 105115). b. Superior cortical endpoint density mapping pArc and TP-SPL. Density projections plotted on both cortical hemispheres. Blue coloring corresponds to nearby TP-SPL endpoints while red coloring corresponds to nearby pArc endpoints. Purple arises in areas of overlap. Viewed from external superior oblique. c. Inferior cortical endpoint density mapping pArc and TP-SPL. Density terminations plotted on both cortical hemispheres. Blue coloring corresponds to nearby TP-SPL endpoints while red coloring corresponds to nearby pArc endpoints. Purple arises in areas of overlap. Viewed from external posterior, inferior oblique and inferiorly. a. and b. depict data for a single subject (HCP 105115). d. And e. depict group sum plots on the MNI brain using the same color conventions as $\mathbf{a}$. and $\mathbf{b}$. 
The two components of the MdLF, like the pArc and TP-SPL dyad, exhibit extensive volumetric overlap in the temporal lobe. Unlike the pArc and TP-SPL, which exhibit slight but distinct differences in their coverages of the fusiform gyrus, the MdLF-SPL and MdLF-Ang are extremely similar in their anterior terminations. Indeed, the aforementioned regions of increased endpoint density in the superior temporal gyrus are found to be largely coextensive (Figures 11c and 11e, Supplementary Figure 5 and 7, Supplementary Figure 3, area 3). As the tracts proceed posteriorly and superiorly through the temporal white matter they begin to exhibit distinct morphologies, with the MdLF-Ang occupying more lateral white matter and the MdLF-SPL occupying more medial white matter. By the time these tracts have reached the parietal white matter they have separated into distinct tracts, with the lateral component (the MdLF-Ang) coursing towards the inferior parietal lobule and angular gyrus, and the medial component (the MdLF-SPL) continuing vertically towards the superior parietal lobule (Figure 11a, Coronal, and Figures 11b and 11d Supplementary Figure 3, areas 6, 7, 8 and 14).
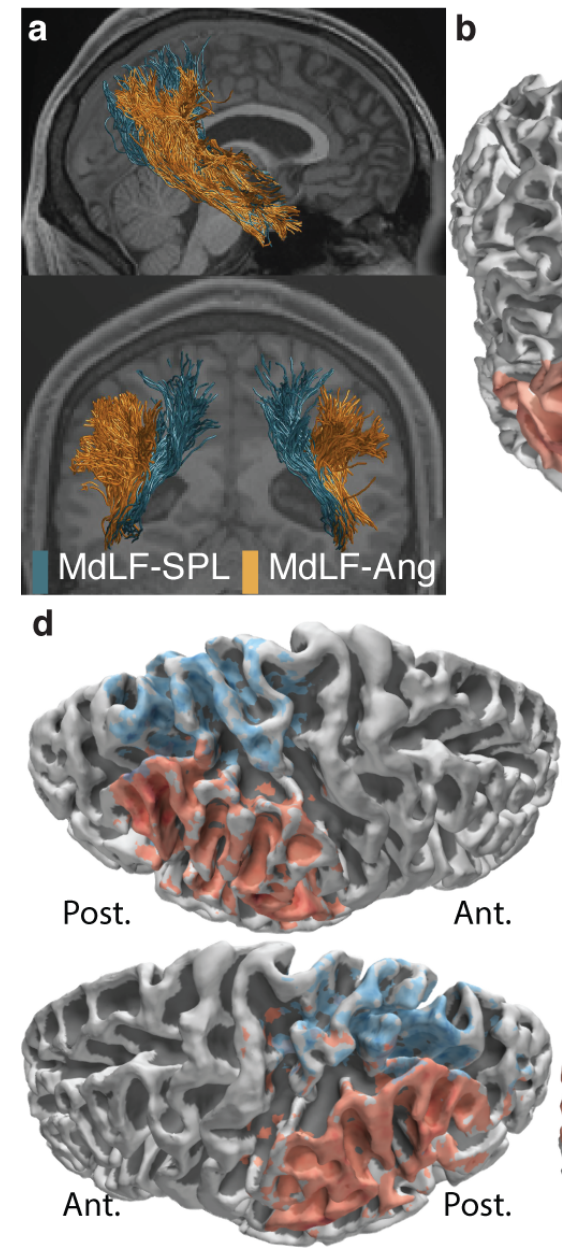
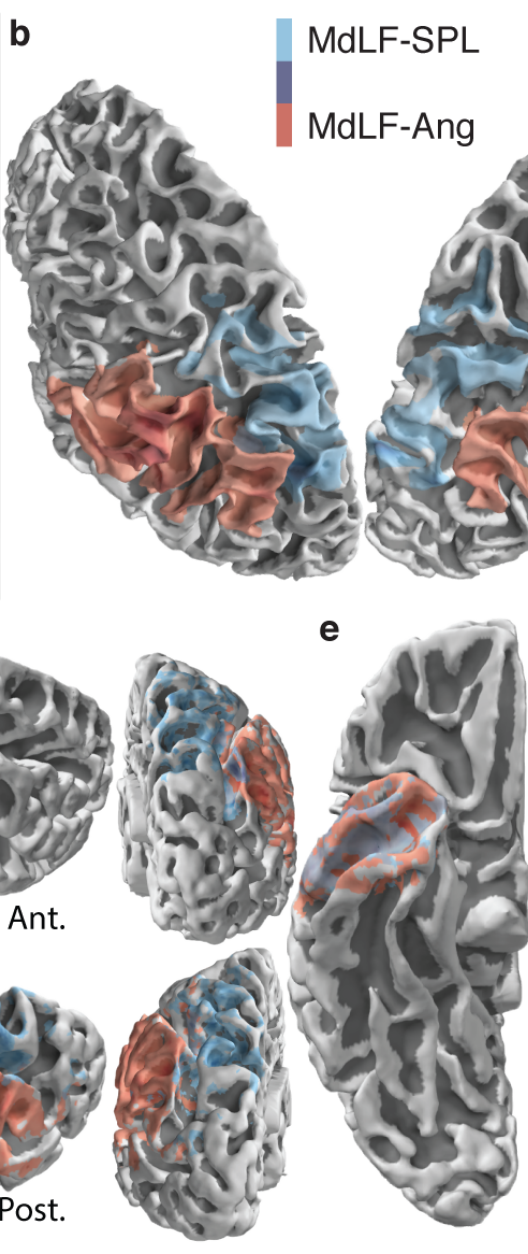
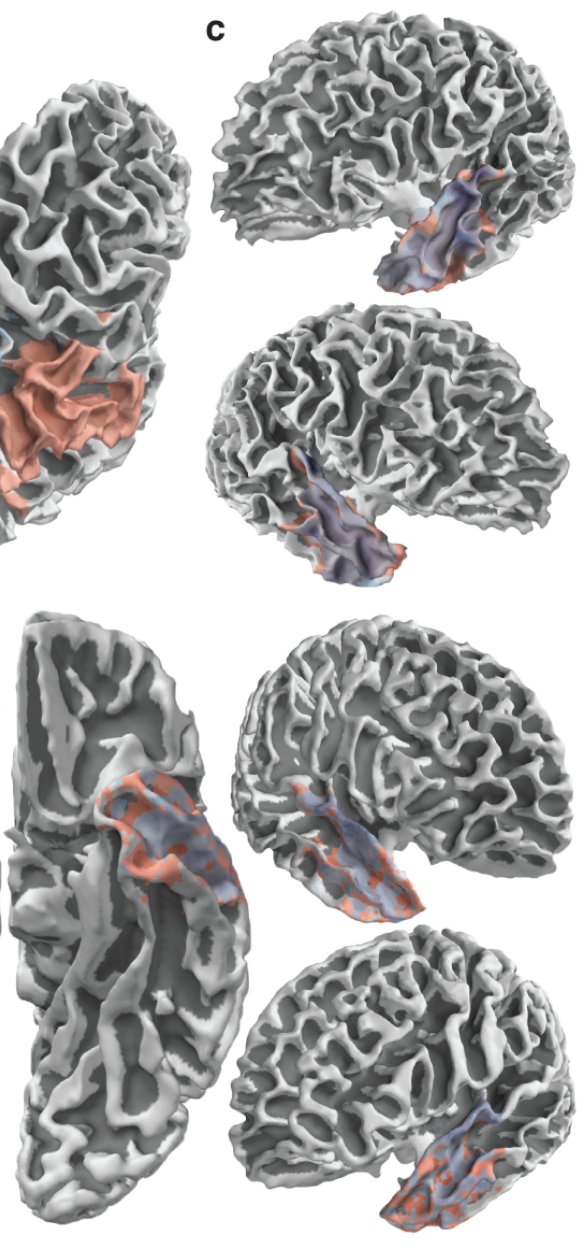

Fig. 11 Relation between the MdLF-SPL and MdLF-Ang cortical termination patterns. a. Two tracts anatomy, MdLF-Ang and MdLF-SPL. Anatomy of the two tracts of interest is plotted over a representative sagittal and coronal brain slice for a single subject (HCP 105115). b. Superior cortical endpoint density mapping MdLF-SPL and MdLF-Ang. Density projections plotted on both cortical hemispheres. Blue coloring corresponds to nearby MdLF-Ang endpoints while red coloring corresponds to nearby MdLF-SPL endpoints. Purple arises in areas of overlap. Viewed from external superior oblique. c. Inferior cortical endpoint density mapping MdLF-SPL and MdLF-Ang. Same convention as Panel c.

External anterior oblique view. a. and b. depict data for a single subject (HCP 105115). $\mathbf{d}$ and $\mathbf{e}$. Compound tract endpoint overlap maps across 8 subjects. Maps depict sum plots across subjects displayed on the MNI template (same color conventions as $\mathbf{a}$ and $\mathbf{b}$; https://doi.org/10.6084/m9.figshare.4223811.v1).

Dorsally overlapping tract terminations. Although the IPS serves as a divisor for the pArc and TP-SPL as well as the sub-components of the MdLF, it also serves as a feature of commonality. In the case of the TP-SPL 
and MdLF-SPL (Figure 12a), both tracts are noted to have their superior terminations medial to this sulcal boundary (Figures 12b and 12d). The apparent discrepancy in the fullness of their termination regions is likely due to the difference in the average number of streamlines per tract (Supplementary Tables $\mathbf{1}$ and 2). The tracts share no overlap in their inferior terminations, owing to the tracts' distinctive termination regions (superior temporal and anterior temporal gyrus for the MdLF-Ang and middle inferior temporal and fusiform gyrus for TP-SPL; Figure 12c and 12e Supplementary Figure 3, areas 1, 3, 16).
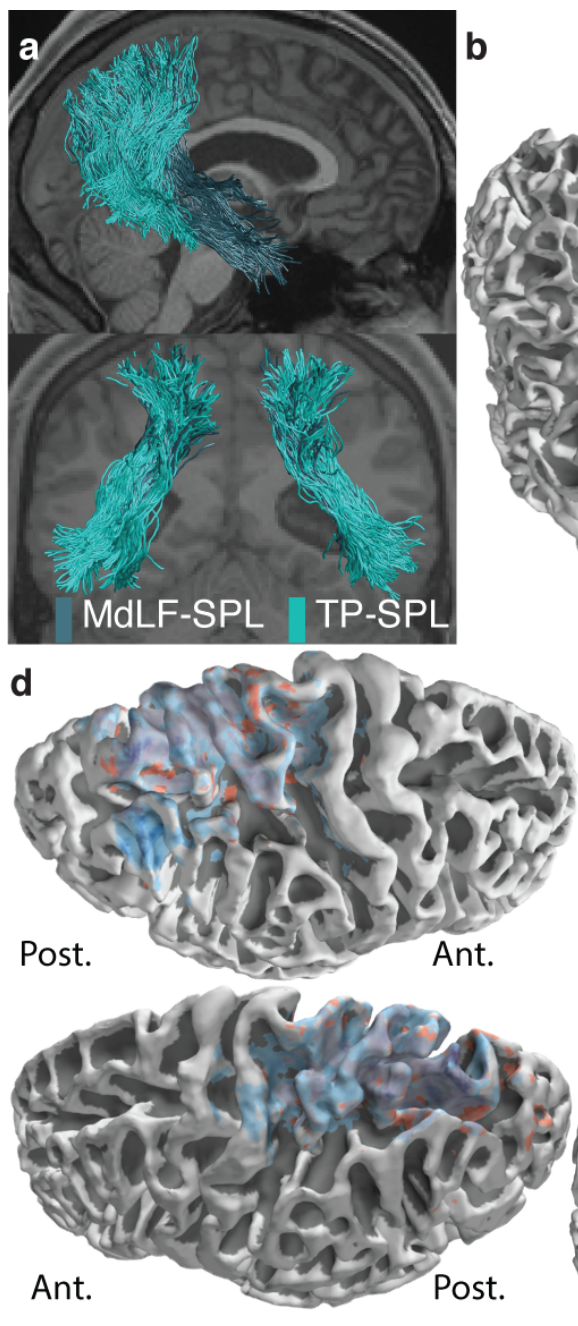
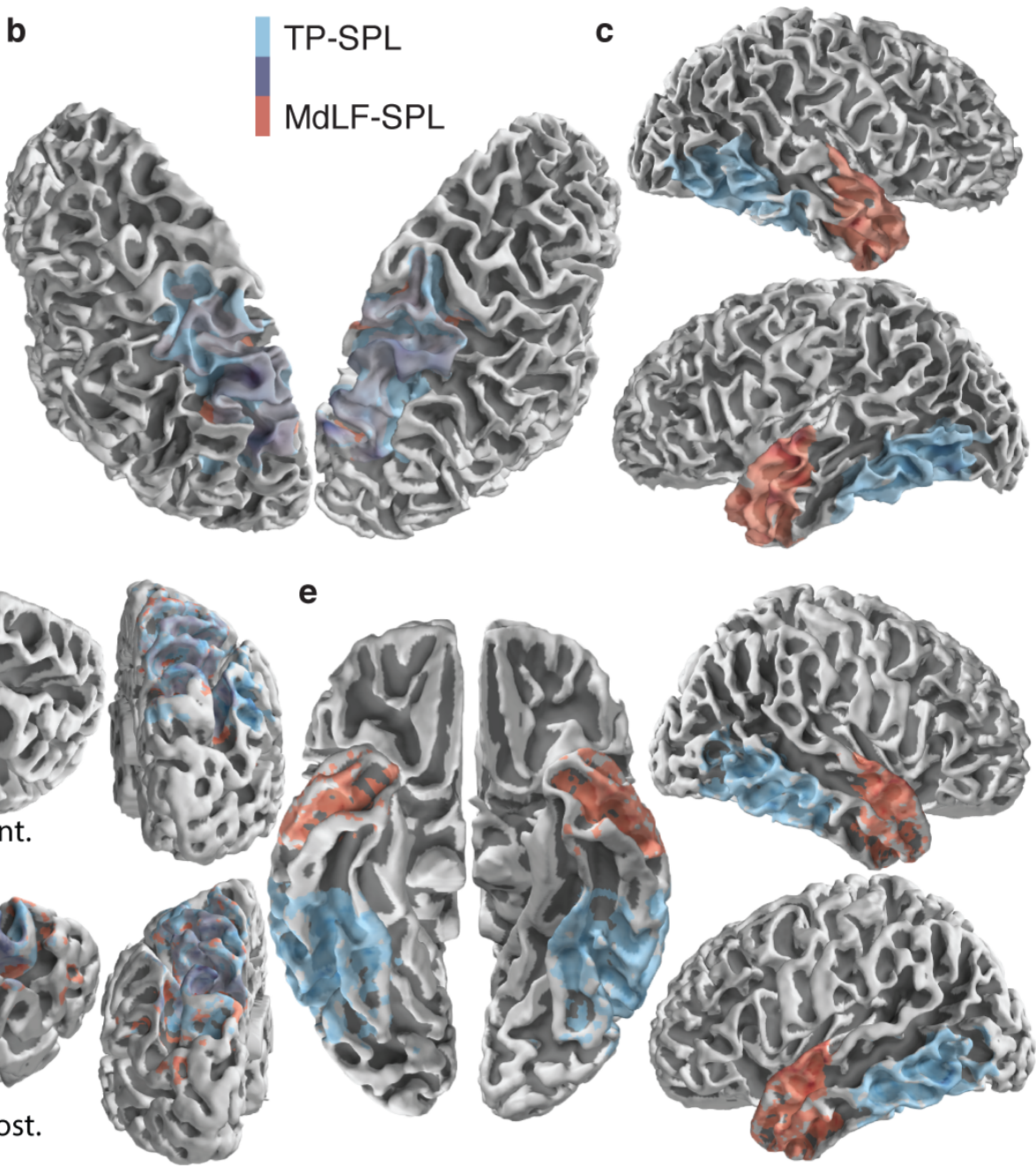

e

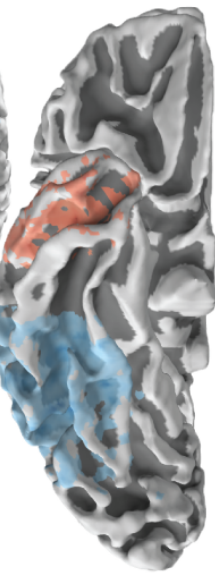

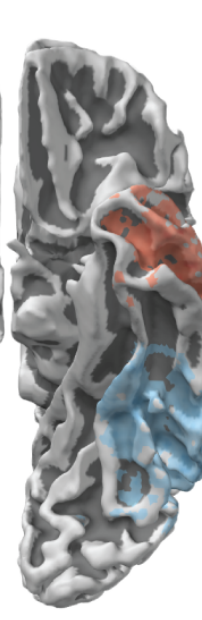

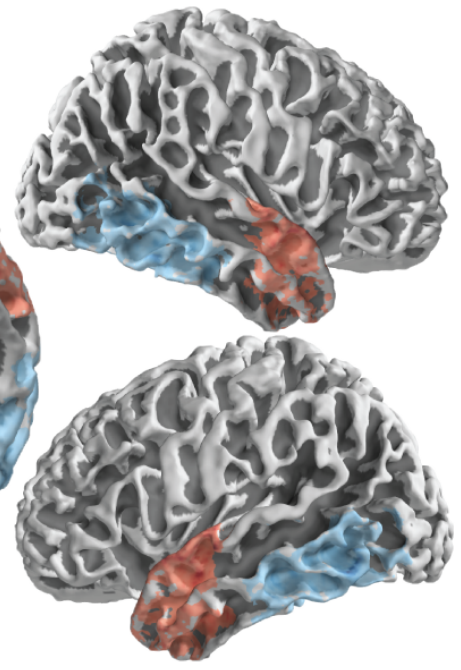

Fig. 12. Relation between the MdLF-SPL and TP-SPL cortical termination patterns. a. Two tracts anatomy, MdLF-SPL and TP-SPL. Anatomy of the two tracts of interest is plotted over a representative sagittal and coronal brain slice for a single subject (HCP 105115). b. Superior cortical endpoint density mapping MdLF-SPL and TP-SPL. Density projections plotted on both cortical hemispheres. Blue coloring corresponds to nearby TP-SPL endpoints while red coloring corresponds to nearby MdLF-SPL endpoints. Purple arises in areas of overlap. Viewed from external superior oblique. c. Inferior cortical endpoint density mapping MdLF-SPL and TP-SPL. Same convention as Panel b. Viewed from orthogonal sagittal. a. and $\mathbf{b}$. depict data for a single subject (HCP 105115). d and e. Compound tract endpoint overlap maps across 8 subjects. Maps depict sum plots across subjects displayed on the MNI template (same color conventions as $\mathbf{a}$ and $\mathbf{b}$; https://doi.org/10.6084/m9.figshare.4223811.v1).

Analogous to the TP-SPL and MdLF-SPL, the pArc and MdLF-Ang exhibit similarities in their dorsal morphology and terminations but significant differences in their ventral terminations (Figure 12a). Specifically, both tracts have extensive termination density in the anterior and posterior angular gyrus (Figures $\mathbf{1 2 b}$ and $\mathbf{1 2 d}$

Supplementary Figure 3, areas 6 and 7), while the MdLF-Ang has its ventral terminations contained within the temporal pole and anterior superior temporal gyrus (Figures 12c and 12e Supplementary Figure 3, areas 3 
and 15) and the pArc has its ventral terminations contained within the the middle and inferior temporal gyri (Figures 12c and 12e Supplementary Figure 3, areas 1 and 3). Closer inspection also reveals that the MdLF-Ang and pArc overlap in a region that may correspond to Wernicke's area. But also differences in endpoint densities, with the MdLF-Ang proceeding further inferior and anterior in Wernicke's area than the pArc.
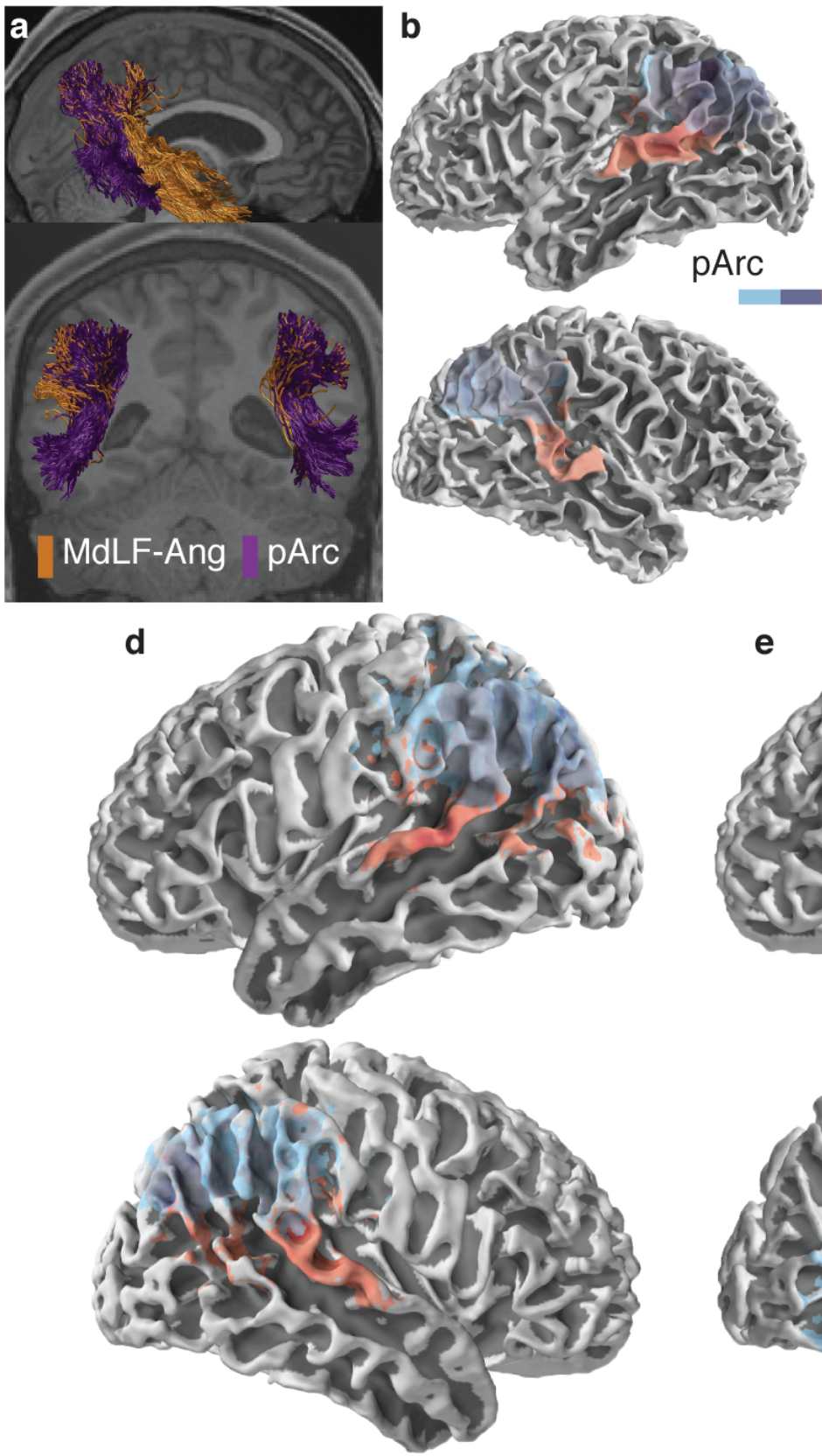

C
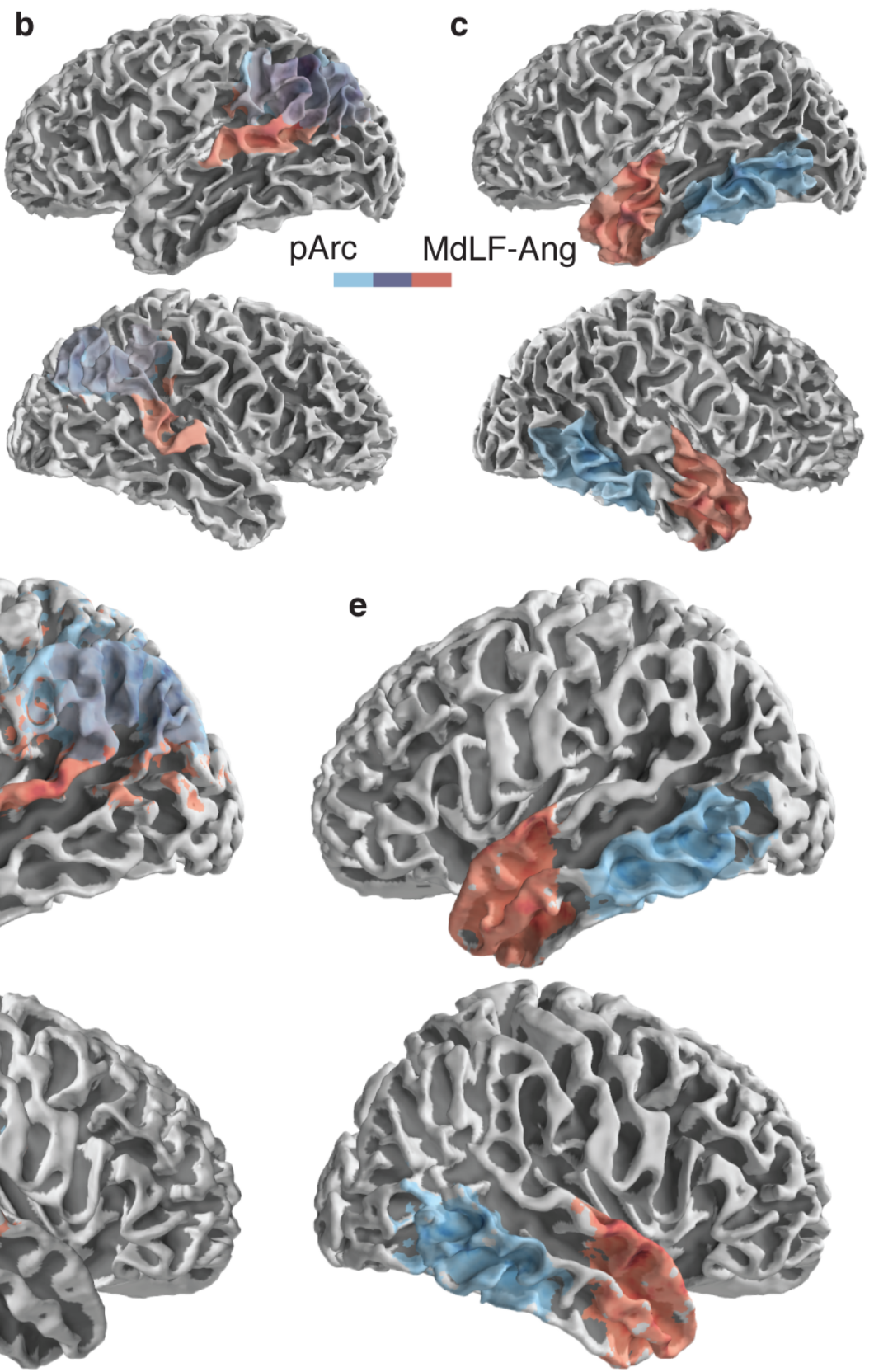

Fig. 13 Relation between the MdLF-Ang and pArc cortical termination patterns. a. Two tracts anatomy, MdLF-Ang and pArc. Anatomy of the two tracts of interest is plotted over a representative sagittal and coronal brain slice. b. Superior cortical endpoint density mapping MdLF-Ang and pArc. Density projections plotted on both cortical hemispheres. Blue coloring corresponds to nearby pArc endpoints while red coloring corresponds to nearby MdLF-Ang endpoints. Purple arises in areas of overlap. Viewed from orthogonal sagittal. c. Inferior cortical endpoint density mapping MdLF-Ang and pArc. Same convention as Panel b. Sagittal view. a. and b. depict data for a single subject (HCP 105115). d and e. Compound tract endpoint overlap maps across 8 subjects. Maps depict sum plots across subjects displayed on the MNI template (same color conventions as a and $\mathbf{b}$; https://doi.org/10.6084/m9.figshare.4223811.v1). 


\section{Comparison of canonical and noncanonical tract terminations.}

Examination of the shared overlap of the Arc with the TP-SPL (Figure 14b and 14d) and pArc (Figure 14c and 14e) reveals that the Arc has a larger endpoint footprint than either the TP-SPL or the pArc (Figure 14b through 14e, yellow). Indeed, the vast majority of both of the pArc and TP-SPL's inferior endpoint mappings are subsumed within the endpoint mapping of the Arc. The posterior and inferior borders of the Arc's endpoint mapping is largely coextensive with the pArc. However, the TP-SPL is observed to have more extensive endpoint mapping in the inferior-medial regions (Figure 14b and 14d, blue). Finally, the Arc is noted to possess endpoints in the anterior temporal lobe the temporal pole, which is found in neither the TP-SPL nor the pArc (Figure 14b through 14e, yellow). In addition to sharing a similar morphology in their temporal terminations, the TP-SPL and pArc have similar, but distinct terminations, in their parietal lobe (See Figure 10b through 10e. The same regions of the parietal lobe also happens to be a major termination region for the SLF (see

Supplementary Figures 5 and 7, Supplementary Figure 3, areas 6, 7, 8, and 14).

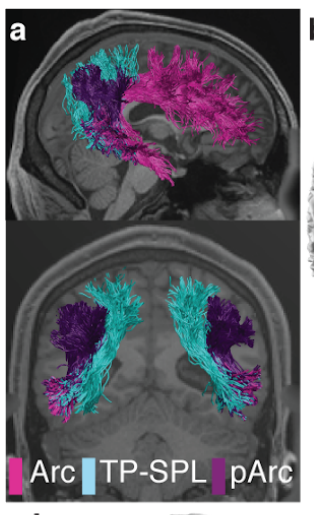

d

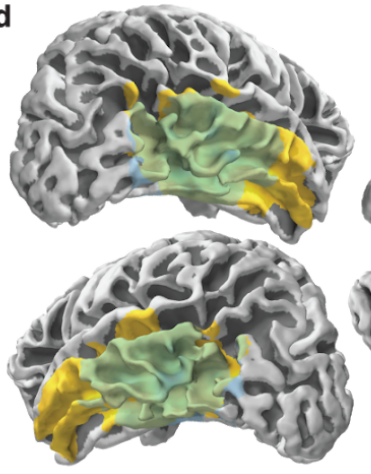

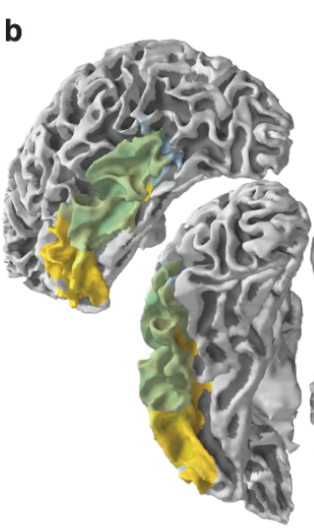
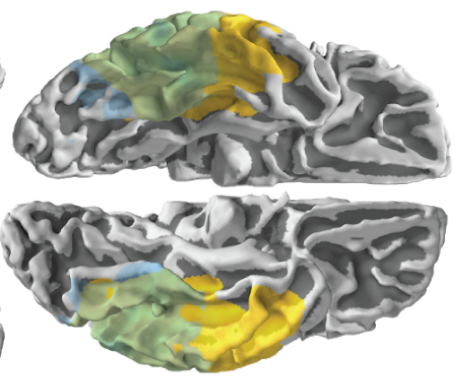

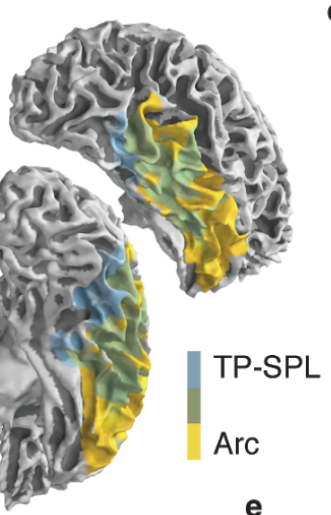

e
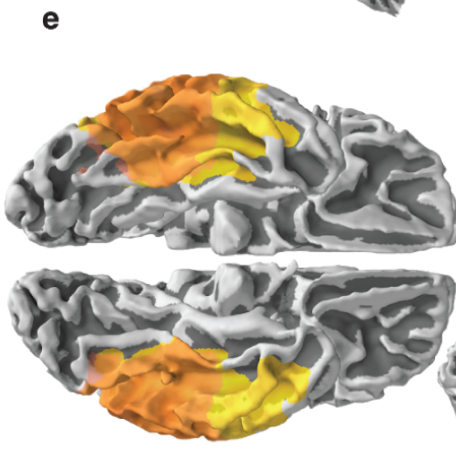
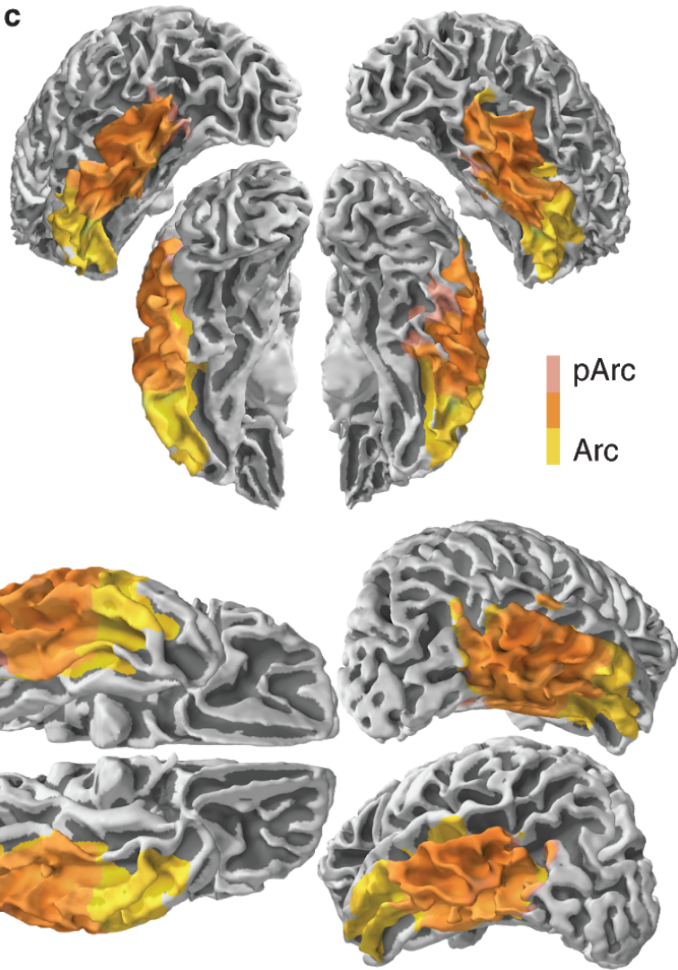

Fig. 14 Relation between the Arc, pArc and TP-SPL cortical termination patterns. a. Anatomies of the three TOls are plotted over a representative sagittal and coronal brain slice for a single subject (HCP 105115). b. Cortical endpoint density mapping Arc and TP-SPL. Blue coloring corresponds to nearby TP-SPL endpoints while yellow coloring corresponds to nearby Arc endpoints. Green arises in areas of overlap. c. Cortical endpoint density mapping Arc and pArc. Density projections plotted on both cortical hemispheres. Red coloring corresponds to nearby pArc endpoints while yellow coloring corresponds to nearby Arc endpoints. Orange arises in areas of tract projection overlap. $\mathbf{a}$. and $\mathbf{b}$. depict data for a single subject (HCP 105115). d and e. Compound tract endpoint overlap maps across 8 subjects. Maps depict sum plots across subjects displayed on the MNI template (same color conventions as $\mathbf{a}$ and $\mathbf{b}$; https://doi.org/10.6084/m9.figshare.4223811.v1).

The TP-SPL, MdLF-SPL and VOF are noted to exhibit several morphological similarities. The preceding discussion of Figure 12 characterizes the relationship between the MdLF-SPL and TP-SPL, while Figure 15 further incorporates the VOF. First, and most obviously, these three tracts connect ventral cortical areas to dorsal cortical areas. The VOF, by definition, does this in the occipital lobe, while the TP-SPL and MdLF-SPL do this for the parietal and temporal lobes. More interestingly though, they all exhibit a progression from lateral 
connective patterns in their inferior regions to medial connective patterns in their superior regions (Figure 15a). Furthermore, the MdLF-SPL and TP-SPL about the VOF in such a way that they appear to form a sheet. This anatomical proximity is more salient when looking at the occipitoparietal arch (Supplementary Figure 3, area 18), which arises at the border of the parietal and occipital lobes. All three tracts share some endpoint density in this region (Figure 15b through 15e, also see Supplementary Figures 5-8).
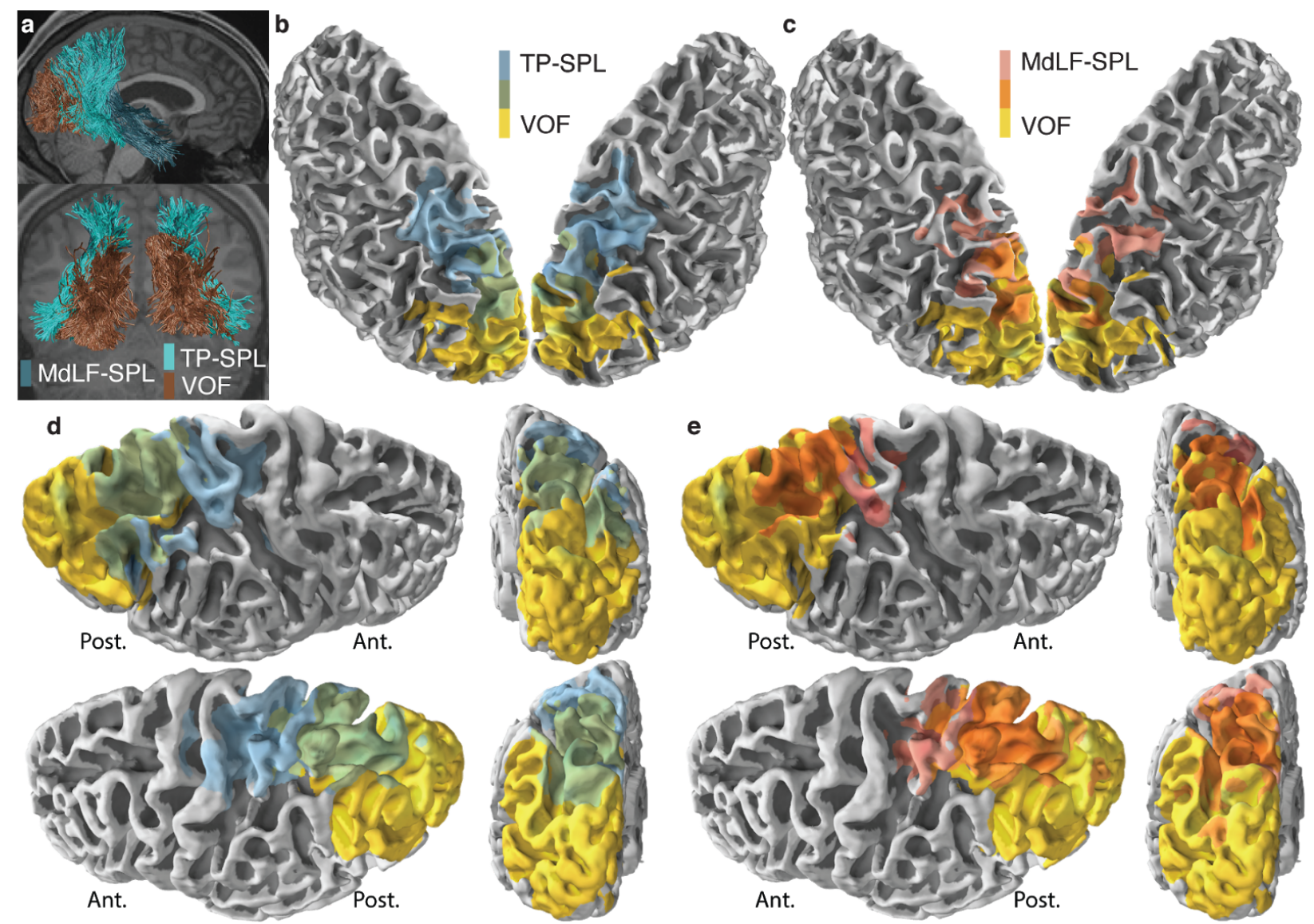

Fig. 15 Relation between the MdLF-SPL, VOF, and TP-SPL cortical terminations patterns. a. Three tracts anatomy, MdLF-SPL, TP-SPL and VOF. Anatomy of the three tracts of interest is plotted over a representative sagittal and coronal brain slice for a single subject (HCP 105115). b. Cortical endpoint density mapping MdLF-SPL and VOF. Density projections plotted on both cortical hemispheres. Blue coloring corresponds to nearby TP-SPL endpoints while yellow coloring corresponds to nearby VOF endpoints. Green arises in areas of overlap. c. Cortical endpoint density mapping MdLF-SPL and VOF. Density projections plotted on both cortical hemispheres. Red coloring corresponds to nearby pArc endpoints while yellow coloring corresponds to nearby MdLF endpoints. Orange arises in areas of overlap. a. and b. depict data for a single subject (HCP 105115). d and e. Compound tract endpoint overlap maps across 8 subjects. Maps depict sum plots across subjects displayed on the MNI template (same color conventions as $\mathbf{a}$ and $\mathbf{b}$; https://doi.org/10.6084/m9.figshare.4223811.v1).

Finally, the MdLF (SPL and Ang) and ILF warrant additional joint-consideration due to the close proximity of their posterior terminations. We begin by looking at Figure 16a and note that the three tracts share extensive volumetric overlap in the anterior temporal lobe, indicating a common origin. However, as the tracts proceed posteriorly, they eventually split and course towards their respective posterior termination areas in the parietal and occipital lobes. Two specific regions exhibit regions of overlap, namely the occipitoparietal arch (Mai et al. 2015) (Supplementary Figure 3, area 18) for the ILF and MdLF-SPL and the extreme posterior angular gyrus for the ILF and the MdLF-Ang (Supplementary Figure 3, area 6). While the border between the MdLF subcomponents is fairly well defined (IPS, Supplementary Figure 3, area 13) the border between the MdLF 
components and the ILF (medial ramus of transverse occipital sulcus for MdLF-Ang and superior ramus of anterior occipital sulcus for MdLF-SPL) may be difficult to definitively identify. We note that, although reported in two separate panels (Figure 16b through e), the two subcomponents of the MdLF (Ang and SPL) can be thought of as forming a continuum with the occipital termination of the ILF.
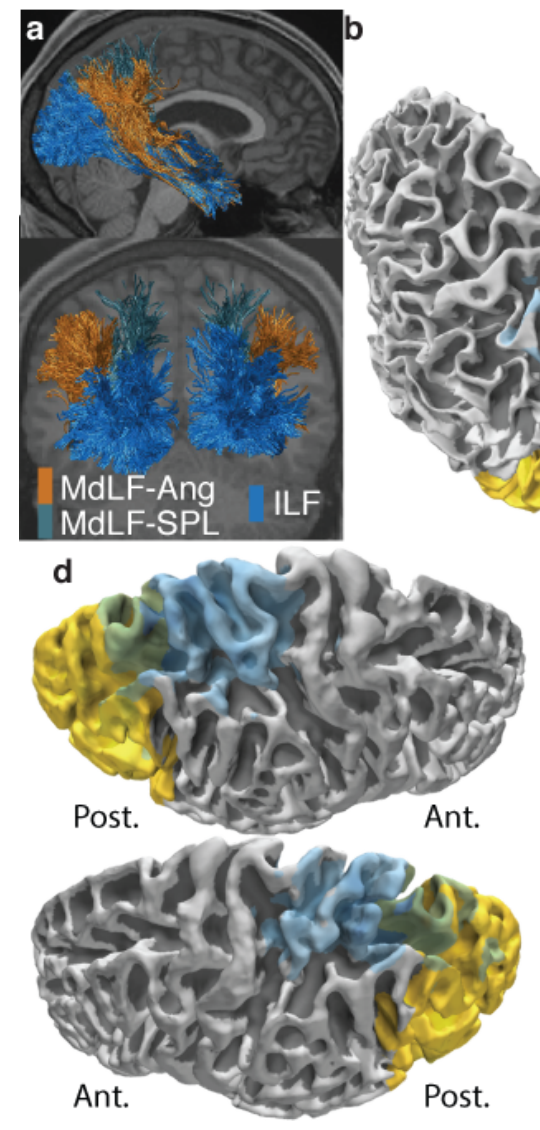
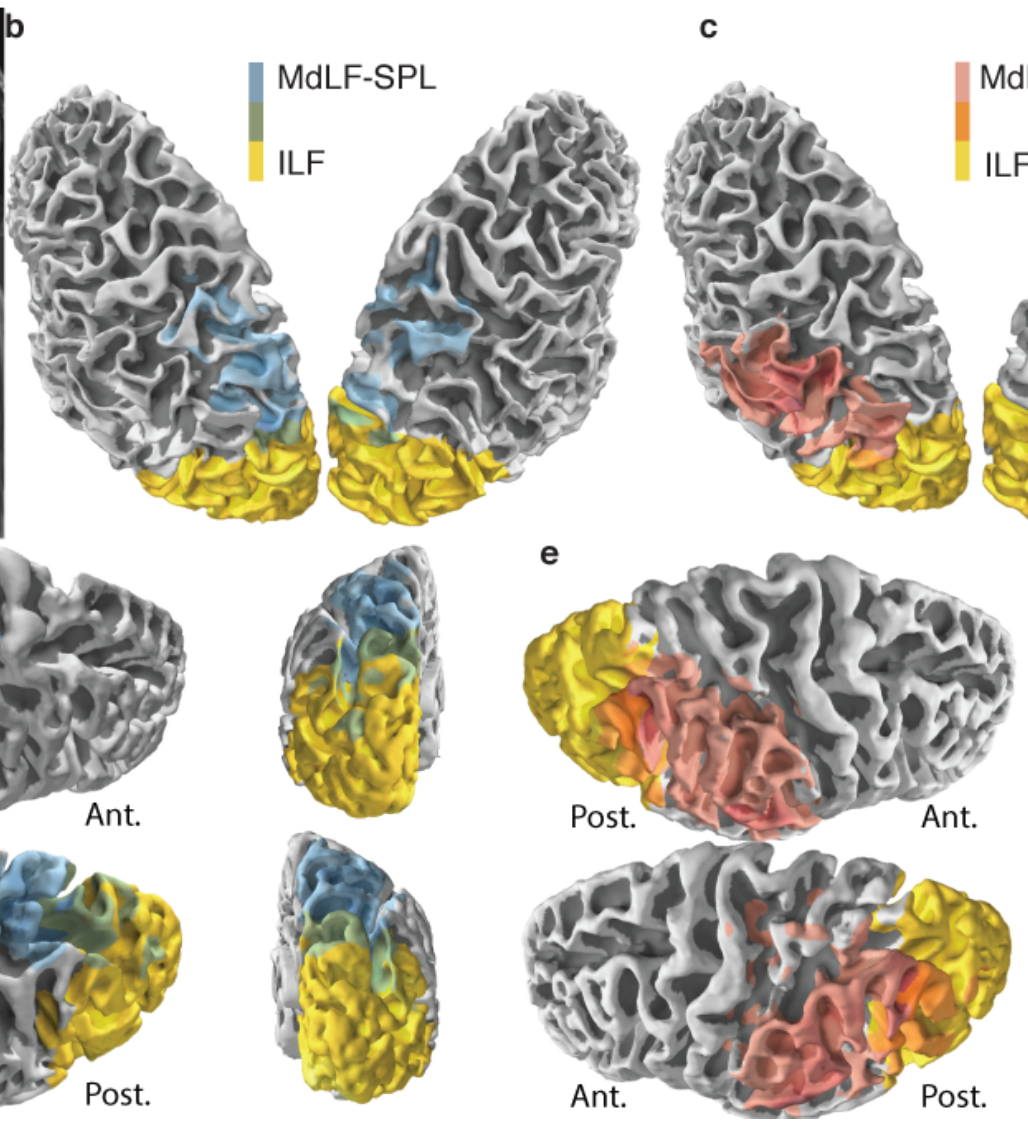

Fig. 16 Relation between MdLF and ILF cortical terminations patterns. a. Anatomy of the MdLF-Ang, MdLF-SPL and ILF. Anatomy of the three tracts of interest is plotted over a representative sagittal and coronal brain slice for a single subject (HCP 105115). b. Cortical endpoint density mapping MdLF-Ang and ILF. Density projections plotted on both cortical hemispheres. Blue coloring corresponds to nearby Mdlf-Ang endpoints while yellow coloring corresponds to nearby ILF endpoints. Green arises in areas of overlap. Viewed from external superior oblique c. Cortical endpoint density mapping MdLF-SPL and ILF. Density projections plotted on both cortical hemispheres. Red coloring corresponds to nearby MdLF-SPL endpoints while yellow coloring corresponds to nearby ILF endpoints. Orange arises in areas of overlap. Viewed from external superior oblique. a. and $\mathbf{b}$. depict data for a single subject (HCP 105115). $\mathbf{d}$ and $\mathbf{e}$. Compound tract endpoint overlap maps across 8 subjects. Maps depict sum plots across subjects displayed on the MNI template (same color conventions as $\mathbf{a}$ and $\mathbf{b}$; https://doi.org/10.6084/m9.figshare.4223811.v1).

\section{DISCUSSION}

Above, we provide an in vivo characterization of several heretofore understudied human white matter tracts. We present anatomical data demonstrating how tracts are organized within the deep white matter as well as putative zones of cortical termination. The contribution of our study is in providing a cohesive, global picture of the relation of commonly studied tracts, the canonical tracts, with less studied tracts. In addition, we provide open source software to segment these tracts automatically in tractographic data sets. This work complements previous reports and methods relating to white matter segmentation and contributes to open science efforts for the sharing of data and methods (Catani and de Schotten 2012; Yeatman et al. 2012, 2014, 2018; Garyfallidis et al. 2014; Takemura et al. 2016b; Wassermann et al. 2016; Weiner et al. 2016). 
The increased availability of data, software, and methods for modeling and segmenting underreported tracts can serve to advance our understanding of the complex arrangement of brain connections (Hagmann et al. 2008; Bullmore and Sporns 2009; Yeh et al. 2018). For example, using modern data and methods a white matter tract between the dorsal and ventral human occipital cortex, the VOF (Yeatman et al. 2013; Takemura et al. 2016b; Wu et al. 2016; Weiner et al. 2016; Lee Masson et al. 2017), has recently been "rediscovered". This result, along with contemporary work on the pArc (Catani et al. 2005; Weiner et al. 2016), have hinted at a more complex white matter architecture in the posterior of the human brain than previously presumed (Hubel and Livingstone 1987; Goodale and Milner 1992; Ungerleider and Haxby 1994; Sakata et al. 1997; Milner and Goodale 2008). Such findings are consistent both with the observation that cortical responses in the ventral and dorsal pathways are suggestive of substantial communication between these areas and with localization of functional specificity outside of cortical regions traditionally associated with responses to different classes of stimuli and actions (Grill-Spector et al. 1998; Steinmetz and Moore 2014; Saber et al. 2015) . Indeed, a more robust understanding of both canonical and non-canonical tracts can, in principle, help elucidate our understanding of information flow in the brain.

We introduced our investigation by reporting that different white matter tracts have received various levels of attention (Figures 1a and 2a). There can be multiple reasons for why this might be. Our goal is to provide clarification of the anatomical features characteristic of these tracts along with methods for segmentation to promote future investigations with a more uniform distribution of attention across these tracts. Below we briefly discuss a few reasons why some white matter tracts might have received less attention.

Disconnection syndromes, white matter tract size and investigative attention. Human white matter tracts have historically received attention due to two broad categories of studies. First, there is the body of work on disconnection syndromes (Catani and Ffytche 2005; Schmahmann and Pandya 2006; Catani and de Schotten 2012; Charcot 2016) wherein the effects of lesions of the cortex or white matter are studied in relation to their impact on behavior in clinical patients. Several reports starting in the mid- to late-19th century demonstrated that lesions within either the cortex or the white matter of the human brain were significantly associated with loss of particular behavioral capacities (Broca 1865; Wernicke 1874; Charcot 2016). These studies where fundamental in establishing a model of the brain where disruptions of connectivity impaired brain function (Geschwind 1965, 1974; Catani and Ffytche 2005; Miller 2010)(Geschwind 1965, 1974; Miller 2010). Second, there are the classical investigations of white matter architecture in putative healthy brains using macroscopic dissection methods (Dejerine and Dejerine-Klumpke 1895; Schmahmann and Pandya 2007). Many of the tracts identified as particularly relevant in these classical investigations are the same we categorize as canonical tracts in the current work (i.e., the arcuate and the SLF). The apparent prominence of these tracts in disconnection syndromes may genuinely be due to their central computational role within the brain -- in short, they truly are more "important". Alternatively, it may that this apparent prominence is merely a consequence of these tracts' larger size (Supplementary Tables 1 and 2) and that brain lesions might be biased towards affecting larger tracts. A hypothetical experiment helps illustrate this possibility.

Imagine an experiment wherein lesions are applied to localized portions of the brain tissue. We begin by choosing a sparse, random pattern of brain locations. For the purposes of our hypothetical experiment, the lesions can be applied either within the white matter or gray matter volume, as the rationale would still hold in either case. This is because larger tracts tend to project to larger cortical regions. Independently of function or importance, the larger white matter tracts, and the capacities they support, have a higher probability of being affected by such random lesions. Such an experiment highlight one possible reason why historical analyses based on lesion studies and dissection methods may have biased us toward the larger, horizontally oriented canonical tracts.

Theory of neuroscience and biases to investigative attention. The german neuroanatomist Theodor Meynert can be credited with highlighting the functional significance and the anatomical organization of the human white 
matter tracts (Schmahmann and Pandya 2006; Catani and de Schotten 2012; Yeatman et al. 2014; Charcot 2016). Meynert advocated a specific conception of white matter architecture with an anatomical classification matching the functional role of three major types of white matter pathways. Commissural fibers were defined as the pathways connecting between the two hemispheres. Projection fibers were defined as vertical white matter pathways connecting cortex to subcortical nuclei and the spinal cord. Association fibers were defined as connecting horizontally cortical regions within a hemisphere (Stricker 1871; Catani and Ffytche 2005; Yeatman et al. 2014; Charcot 2016). Meynert's theoretical framework influenced many subsequent studies of the cerebral white matter. The theoretical preference in defining association fibers as running horizontally and projection fibers as running vertically can be interpreted as a potential explanation for why non-canonical, vertically oriented tracts, might have received reduced investigative attention over time. The recent re-discovery of the vertical occipital fasciculus (VOF; (Yeatman et al. 2014)) has spurred interest in the possibility that the prevailing norms assuming horizontally travelling associative fibers might be limited. Here, we have provided additional clarity on the structure of several non-canonical tracts that can nonetheless be interpreted as being associative in nature.

In sum, both the relatively smaller size and shorter length of the non canonical tracts, along with the established paradigms governing the study of the brain connections may have jointly contributed to reduced investigative attention being paid to several of the tracts characterized and clarified in the present work. Below we briefly mention some of the debates and differences in definitions and naming of the non-canonical tracts that are still found in the literature. We hope this work will be helpful establishing a reconciled model of the human white matter that can inform future investigations and provide some degree of clarity on the white matter organization (Wandell 2016).

What is a white matter tract? Any work attempting to clarify white matter (Takemura et al. 2016b; Wandell 2016; Rokem et al. 2017) by characterizing the anatomy of white matter tracts (either utilizing ex vivo or in vivo methods) can raise general questions regarding the definition of a white matter tracts per se. What should be the "anatomical" definition of a white matter tract? When should a bundle of neuronal fibers be elevated to the level of tract? When instead it should be considered as part of another (larger tract)? In the present work, we present no previously unreported tracts here.

We believe that, in principle, any set of fibers that (1) cohesively and smoothly travel together and (2) connect regions with similar functional profiles can be clustered together into a single tract and named as such. Yet, historically tracts were named only on anatomical grounds (Obersteiner 1890; Dejerine and Dejerine-Klumpke 1895; Gray 1918). Because of this, several of the major white matter tracts (i.e. Arc, SLF) are likely to communicate multiple functions and connect a variety of areas. Indeed, given the above definition of tract, concerns about hypersegmentation of the white matter tissue into too many, small tracts, can be brought up. We believe that we are currently far from hypersegmentation as there are likely to be more functionally distinct white matter tracts than we currently name and understand. Indeed, recent work has proposed that some of the established tracts, such as the pArc, should be further subdivided and renamed (Panesar et al. 2019). Likewise, in the present work, and following the work of Kamaili et al. (Kamali et al. 2014a, b), we clarify the distinction between the posterior arcuate (pArc), which travels lateral to the Arcuate fasciculus, and the temporo-parietal connection (TP-SPL), which travels medial to the Arcuate fasciculus (Supplementary Figure 1).

A different question that follows the discussion on the definition of a white matter tract is novelty; when is a tract new? In the present work, we discuss tracts that have been previously reported elsewhere. Recently, tracts have been defined also as "rediscovered" (Gabrieli 2009; Thomason and Thompson 2011; Thompson et al. 2014; Ogawa et al. 2014; Yeatman et al. 2014; Gomez et al. 2015; Allen et al. 2015; Ajina et al. 2015; Leong et al. 2016). Importantly, tracts have been also newly named, for example when a larger tract has been subdivided into smaller one, as has been the case with the Arcuate and pArc (Catani et al. 2005), the pArc itself 
(Panesar et al. 2019). Other tracts have been also reported to provide novel insights in to brain connectivity (Catani et al. 2013; Ajina et al. 2015; Panesar et al. 2019; Sani et al. 2019).

Clarity on the distinction between the Temporo-Parietal Connection and the Posterior Arcuate. The temporal parietal connection (TP-SPL) is one of the least studied tracts among the ones we discuss here (Figure 2b). Previously the inferior portion of the TP-SPL has been shown to follow the posterior curvature of the arcuate fasciculus, but proceeding vertically into the Superior Parietal Lobule (SPL) instead of turning anteriorly as the arcuate does. There is a similarity between the paths taken by TP-SPL and the posterior arcuate (pArc) and previous work has noted such similarity (Wu et al. 2016). Indeed the two tracts have been characterized as being part of a single structure (Kamali et al. 2014a). The present results clarify the notion that these two structures can be meaningfully separated in virtue of their difference in inferior and superior cortical terminations. We further provide novel results which show that the TP-SPL terminates near the fusiform gyrus (Figures 7, Supplementary figure 5 and 7, Supplementary Figure 3, area 16), which in turn supports previous claims regarding the temporal terminations of the TP-SPL (Wu et al. 2016).

Clarity on the distinction between the Posterior Arcuate and Vertical Occipital Fasciculus. The posterior Arcuate (pArc) has been depicted as a single, fairly unified, laterally concave body (Catani et al. 2005). These early results were mostly based on virtual dissections using early tractography methods (deterministic tracking; see reviews for details on these methods (Wandell 2016)(Wandell 2016; Rokem et al. 2017). At the time, it was reported that the pArc connected between regions of the medial and inferior temporal gyri and the angular gyrus. These findings were consistent with post mortem dissections results (Lawes et al. 2008;

Fernández-Miranda et al. 2008). The present work uses a more recent tractography method (Ensemble Tractography (Takemura et al. 2016a). The more recent methods reportedly can map more tracts with a wider anatomical shapes, than the early methods (Takemura et al. 2016a). Yet, beside the remarkable differences in tractography methods and the anatomical differences that the methods can generate, the results reported in the current work are consistent with those of the early reports mentioned above. One exception is the extent of the cortical projection of the pArc; we find that extent to be potentially much larger than previously considered (Figure 6).

More recently, researchers have taken the care to distinguish between the pArc and VOF (Weiner et al. 2016). In the past, there have been various instances of an apparent confusion between two these tracts (Homola et al. 2012; Martino and García-Porrero 2013; Bartsch et al. 2013). Our results support the notion of a clear anatomical separation between these two tracts. As evident in their naming convention, the VOF is constrained within the occipital lobe, while the pArc does not enter the occipital lobe. We believe that the current results are consistent previous reports, and provide additional clarification on this issue (Weiner et al. 2016).

Clarity on the definition of the Middle Longitudinal Fasciculus. The MdLF is another of the understudied, non-canonical tracts (Figure 2b). We note that we adhere to a definition of MdLF that is based upon a consensus drawn from across the literature in the human brain (Makris et al. 2009; Menjot de Champfleur et al. 2013; Maldonado et al. 2013; Wang et al. 2013; Kamali et al. 2014b). We clarify the underreported characteristics of the anatomy of this tract, along with its cortical terminations zones and relation to other tracts such as the ILF and pArc, which have been previously confused with the MdLF because of potential overlap of cortical termination zones.

A number of reports have consistently indicated that the MdLF projects to the anterior superior temporal gyri (Makris et al. 2009; Menjot de Champfleur et al. 2013; Maldonado et al. 2013; Wang et al. 2013; Kamali et al. 2014b). Yet, there exists less consensus about the MdLF's posterior terminations. In several cases, researchers have argued that the MdLF either terminates in the angular gyrus only (Makris et al. 2009; Turken and Dronkers 2011; Menjot de Champfleur et al. 2013; Martino et al. 2013a; Maldonado et al. 2013), in the superior parietal lobule only (Wang et al. 2013; Dick et al. 2014), or in both (Makris et al. 2013a, b; Kamali et al. 2014a). We report 
evidence for two aspects of this tract terminating in the angular gyrus and superior parietal lobule respectively (see Figures 8, 9, and 11).

We further note that it is has been difficult to unequivocally distinguish the MdLF from the nearby tracts such as the ILF and pArc. Some previous reports appear to have confused anatomical features of MdLF and pArc. Finally, we note that some characterizations of the pArc can make the tract appear to overlap with the MdLF (Catani et al. 2005; Weiner et al. 2016; Budisavljevic et al. 2018). Our results show that MdLF and pArc as well as the MdLF and the ILF can be clearly separated and show distinguishing anatomical features (see Figures 13 and 16).

A positive outlook at understanding white matter. There have been long-standing debates about the nature of the anatomy of brain structure and function (Gall 1810; Flourens 1846; Zola-Morgan 1995; Haxby et al. 2000; Kanwisher 2010; Yeatman et al. 2014; Fang et al. 2018; Pestilli 2018). Over the years, investigators using different methodologies and theoretical perspectives - from anatomical dissection and tracing methods to virtual dissections and neuroimaging - have come to argue about the the degree of functional specialization, nomenclature, and anatomical characteristics of particular components of both the gray and white matter of the brain. As such, the modern methodological debates on the subject of tractography and white matter mapping are not an entirely new phenomenon. Among these modern debates, there are recent reports highlighting possible failures of tracking methods when using simulated data (Maier-Hein et al. 2017), or in specific brain locations (Thomas et al. 2014; Reveley et al. 2015), and even debates on the overarching structure of the white matter organization (Wedeen et al. 2012; Catani et al. 2012).

Despite the recent controversies about the limitations of in vivo tractography, we remain optimistic about the potential for impact. This is especially the case when good care is taken to look for convergent evidence from different measurements modalities and across mapping methods. In our case, we took great efforts to ensure that our segmentations and white matter ontology reflected previous anatomical work and to clarify when convergent evidence was lacking and further investigation will be necessary (see Supplementary Figure 1). Indeed, we find a great degree of consilience between the findings of traditional anatomical dissections and modern neuroimaging methods and that we agree on the significance of major associative white matter tracts (Mori et al. 2005; Schmahmann and Pandya 2006; Catani and de Schotten 2012). Importantly, in addition to establishing models of anatomy, tractography methods have the unique benefit of allowing investigators to study the human brain in vivo. In vivo automated segmentation methods, such as the one presented here (https://doi.org/10.25663/bl.app.46), allow multiple investigators to reproduce or extend previous mapping results. The tissue properties of tracts mapped in vivo can be also studied in their relation to individual-level phenotypes, both in healthy individuals and in states of disorder (Gabrieli 2009; Thomason and Thompson 2011; Yeatman et al. 2011; Thompson et al. 2014; Ogawa et al. 2014; Gomez et al. 2015; Allen et al. 2015; Ajina et al. 2015; Leong et al. 2016).

We have yet to fully unravel the precise role played by the human white matter in human cognition and perception. Neither the structural organization nor the functional role of white matter are completely understood. Indeed, only recently have we become aware of the critical role that white matter plays in establishing successful, healthy, trajectories across long-term life events such as development, learning and in the aging brain. Far more research will be necessary to clarify the functional significance of the human white matter for human behavior. For now we must content ourselves with moderate, incremental improvements in our understanding of this tissue which comprises up to 50 percent of the total brain volume: the white matter. (Zhang and Sejnowski 2000; Fields 2008b; Ventura-Antunes et al. 2013; Wandell 2016).

In this paper we set out to advance the understanding of white matter by better characterizing some of the more understudied, but nonetheless important, white matter structures in the posterior of the human brain - the TP-SPL, the two MdLF tracts, the pArc and VOF. Without these tracts, our understanding of full human visual white matter system is incomplete. 


\section{METHODS}

Data sources. Data and a pipeline developed to process the data according to the presented results is made openly available using the open services model and cloud computing resources at https://brainlife.io (see Table 1). Below we describe the acquisition parameters of the original data used for all our analyses. In short, we utilized data from two published sets the Stanford diffusion data (STN (Pestilli et al. 2014; Rokem et al. 2015)) and the Human Connectome Project data (HCP 3T; (Van Essen et al. 2012; Glasser et al. 2013; Sotiropoulos et al. 2013)). Data collection was approved by the respective Institutional Review Boards (IRBs) of the University of Washington, Saint Louis (HCP dataset) and Stanford University (STN dataset). All participants provided written informed consent to participate in the project.

Stanford data set (STN). Subjects for the STN data set were collected at the Center for Cognitive and Neurobiological Imaging at Stanford University under the supervision of B. Wandell. We used data collected in 4 males using a 3-Tesla General Electric Discovery MR 750 (G.E Healthcare, Chicago, IL), equipped with a 32-channel head coil (Nova Medical, Boston, MA). Diffusion data was collected at 96 diffusion-weighting directions, and this process was done twice during each individual's scan session. Dual-spin echo diffusion weighted sequences (Reese et al. 2003) were used with full head coverage. The standard electrostatic repulsion equation was used to orient diffusion sensitization uniformly on the surface of an idealized sphere. Voxel resolution was set to $1.5 \mathrm{~mm}$ isotropic resolution, with a diffusion gradient strength of $2,000 \mathrm{~s} / \mathrm{mm}^{2}$ (TE $=96.8$ $\mathrm{ms})$. Data were acquired using two excitations $\left(\mathrm{n}_{\mathrm{ex}}=2\right)$ then averaged in k-space. Ten unweighted diffusion images $(b=0)$ were measured at the start of each scan. These were used to calculate the SNR across repeats, which was found to be greater than 20 in all cases (SNR = mean / s.d. of the $b=0$ measures). A more detailed description of data acquisition and preprocessing can be found in preceding work (Pestilli et al. 2014; Rokem et al. 2015; Takemura et al. 2016b).

Human Connectome Project dataset (HCP 3T). For additional validation of our results, we repeated all analyses also on data from the Human Connectome Project's 3T data set by WU-Minn HCP Consortiums (Van Essen et al. 2012; Glasser et al. 2013; Sotiropoulos et al. 2013). Four subjects (105115, 110411, 111312, 113619) from the Human Connectome Project's $3 T$ collection were used. These data were originally collected with three diffusion gradient strengths $\left(1,000,2,000\right.$ and $\left.3,000 \mathrm{~s} / \mathrm{mm}^{2}\right)$ and 90 diffusion directions per gradient strength, with $1.25 \mathrm{~mm}$ isotropic resolution. We extracted the 2,000 shell and performed all analyses on this reduced data set. Data were preprocessed by WU-Minn HCP Consortiums using methods that are described in Sotiropoulos et al. (2013). A more detailed description of further data analyses can be found in preceding works (Pestilli et al. 2014; Rokem et al. 2015; Takemura et al. 2016b, a; Caiafa and Pestilli 2017).

Cortical Parcellation and White Matter Region Identification. The cortical surface was segmented into multiple regions using standard automatic parcellation methods (Fischl 2012). The Destrieux 2009 Atlas (Destrieux et al. 2010) was applied, generating a 152 region parcellation of the gray matter. The gray matter mask used in the cortical termination step was generated in this process. The brain white matter was also identified using this method and was used for tracking (see tract generation section). Below (Methods: White matter tracts segmentation), we also describe how multiple regions from this atlas were used to segment individual white matter tracts.

Repeated Measure Ensemble Tractography. In order to segment the tract of interest (TOI), we generated ten repeated-measure, whole brain ensemble tractography connectomes for each subject (Takemura et al. 2016a). Each repeated-measure was obtained using an independent and pseudo-randomly chosen set of fascicle seeds (Tournier et al. 2012). These repeated measures were used to determine the consistency and reliability of segmentation and other quantitative measures (see error bars in Figures 3A, B and C). Fiber tracking was conducted using MRtrix (Tournier et al. 2012), after generating a white matter mask to delineate the seed region, streamline were seeded from pseudo-random locations within this white matter mask. The tracking used constrained-spherical deconvolution (CSD; (Tournier et al. 2007; Descoteaux et al. 2009) combined with 
probabilistic tractography. This was applied across a range of CSD harmonic orders $\left(L_{\max }=2,4,6,8,10\right)$ and repeated ten times for each harmonic order. A step size of $0.2 \mathrm{~mm}$ was used along with a minimum curvature radius of $1 \mathrm{~mm}$. Additionally streamlines were excluded if they were shorter than $10 \mathrm{~mm}$. 500,000 streamlines were generated at each $L_{\max }$ setting. Within each $L_{\max }$ setting ten replications were performed. From each of these 500,000 streamline whole brain connectomes a random subsample of 100,000 were selected and incorporated into an ensemble. It has been shown that different tracking methods better characterize different white matter tracts (Takemura et al. 2016a). We replicated this previous finding with our tracts and exploited the different properties of each tractography model to improve the anatomical representation of our tracts.

Furthermore, our use of repeated measures allowed us to derive a measure of consistency and error when performing quantitative analysis; error bars in Supplementary Figure 3 indicate one standard deviation across 10 repeated measures tractography.

Tractography Evaluation. A key component of our effort in this investigation was the establishment of quantitative norms for these tracts. This means establishing their canonical size (volume and length) and the variation in these properties. The application of the Linear Fascicle Evaluation (LiFE) algorithm (Pestilli et al. 2014) allowed us to optimize the candidate connectomes and thereby obtain a quantitative measure of how much a given fiber tract improved the fit of our model to the data. Specifically, this method estimates how much each streamline in the candidate connectome predicts the diffusion signal of the measured data, on a voxel-wise basis. This amount is represented by the streamline weight, a property ascribed to individual streamlines, and can manifest as a value of 0 , indicating no contribution towards predicting the diffusion signal. Approximately 165,000 of the 500,000 streamlines per HCP candidate connectome are routinely found to successfully contribute to the measured diffusion signal (i.e., they are assigned non-zero weight values by the LiFE method). In the case of STN candidate connectomes approximately 110,000 of the 500,000 streamlines were found to contribute. This quantity of validated streamlines (and the disparity between data sets) has been linked to the quality of scan data (Caiafa and Pestilli 2017). The streamline weights were subsequently used to determine which streamlines should be featured in anatomical depictions (only those with non zero weightings are plotted in all figures), and as inputs in the virtual lesioning quantitative analysis.

Tract Statistical evidence. In order to quantify the the statistical evidence for a white matter tract, we computed the degree to which the diffusion data supported the existence of the tract by using the "virtual lesion" method (Honey and Sporns 2008; Pestilli et al. 2014). In short, a virtual lesion compares the error a connectome model exhibits when predicting the measured diffusion signal, with and without a TOI in the model. In our analyses, we computed the error (the root-mean-squared error) in predicting the demeaned diffusion signal in all voxels occupied by the tract. To do this we identified all streamlines that intersect voxels occupied by the TOI. The voxels traversed by the TOI are defined as the tract volume, while streamlines intersecting the same voxels are defined as the path neighborhood (Wedeen et al. 2012). The error associated with the virtual lesion method is computed within the tract volume voxels. The model error measured in this volume results from the shared contribution of the TOI and path-neighborhood streamline predictions. To perform virtual lesions we removed a TOI from the LiFE model and computed error in predicting the diffusion signal. This generated two distributions of errors in predicting the diffusion signal in the tract volume voxels; one with the TOI and the neighborhood the other with the neighborhood but no TOI. The increase in error in predicting the diffusion signal was computed by comparing these two distributions using multiple measures the earth mover's distance (EMD; (Rubner, Y., Tomasi, C. \& Guibas, L.J. 2000)) - more details about this procedure can be found in previous work (Pestilli et al. 2014; Gomez et al. 2015; Takemura et al. 2016b, a; Leong et al. 2016; Caiafa and Pestilli 2017; Uesaki et al. 2018).

White Matter Tracts Segmentation. A total of fifteen white matter tracts were segmented from 80 (8 subjects * 10 replications ) whole-brain connectomes. Segmentation of 11 major fiber tracts was achieved using Automated Fiber Quantification (AFQ; (Yeatman et al. 2012)). AFQ segments tracts from a white matter atlas (Wakana et al. 2007) using a combination of a refined 2-ROI tract identification method combined with an atlas 
of the human white matter (Mori et al. 2005). Although, it was originally designed to be used in combination with deterministic tractography, it has been successfully used recently in combination probabilistic tractography (Pestilli et al. 2014; Gomez et al. 2015; Leong et al. 2016; Takemura et al. 2016a, 2017; Caiafa and Pestilli 2017; Uesaki et al. 2018). The VOF was segmented using the addition to AFQ that uses a single ROI methodology, along with additional exclusion criteria, and has been described in previous work (Yeatman et al. 2014).

Here, we further developed an automated methodology for segmenting the pArc, MdLF-Ang, MdLF-SPL, TP-SPL. Our method utilizes ROIs from Freesurfer's Destrieux 2009 parcellation (Destrieux et al. 2010) in a manner similar to the White-Matter Query Language (WMQL; (Wassermann et al. 2013, 2016)). In addition to criteria described in the WMQL, we added additional criteria in order to ensure robustness against false positive streamlines (Maier-Hein et al. 2017) and thereby ensure accurate tract identification. Supplementary Table 3 details the specific criteria identified by our method to segment the MdLF-SPL, MdLF-Ang, TP-SPL, and pArc. Table columns under the "WMQL-like Endpoint inclusion criteria" heading (Supplementary Table 3, green highlight) correspond to inclusion ROIs which are used to identify candidate streamlines by their endpoints. The latter two columns, labeled "Anatomically informed streamline inclusion criteria" (Supplementary Table 3, blue highlight) correspond to additional non-endpoint criteria, necessary to segment a tract. These criteria go beyond what can be done using WMQL. For example, the segmentation of the MdLF-Ang also requires that all fibers mid-points be positioned above the Thalamus. This approach is necessary, particularly with probabilistic tractography, as merely using endpoint criteria is insufficiently specific to ensure accurate streamline identification. Indeed, in our early investigations we occasionally noted that several anatomically inaccurate or biologically implausible streamlines were identified as being part of a segmented tract when these additional criteria were not used. The thoroughly commented code implementation used to generate the data reported here is openly available: https://github.com/brainlife/Vertical Tracts.

Open services and software for automated tract segmentation and reproducible neuroscience. We provide the full set of scripts and code describing a processing pipeline that can be used for processing new dMRI data to segment the non-canonical tracts described here. In addition, we also provide web services that allow processing new data utilizing the algorithms used to generate the results reported above. Web services are made available at https://brainlife.io, the open source code implementing the services is available at www.github.com/brainlife. 


\section{Service method \\ Brain registration \\ Data files normalization \\ Shell splitting \\ $\mathrm{dMRI}$ data preprocessing \\ Brain parcellation \\ Tractography \\ White matter tracts segmentation}

Open cloud services

https://doi.org/10.25663/bl.app.16

https://doi.org/10.25663/bl.app.4

https: / /dai.org/10.25663/bl app.17

https: / /dei.erg/10.25663/blapp.3

https://doi.org/10.25663/bl.app.0

https://dei.erg/10.25663/bl.app.33

https: / /doi.org/10.25663/bl.app.46

Table 1. Examples of methods and open services code implementing the processing pipeline to take new dMRI data to segment the pArc, TP-SPL, MdLF-SPL, MdLF-Ang, and VOF

Cortical termination mapping and quantification. We analyzed the cortical termination endpoints of streamlines in each hemisphere in order to visualize both cortical projection extent and overlap between tract pairings. The fun Precursor versions of this code, specific to density volume creation, were used in previous works (Takemura et al. 2016b; Uesaki et al. 2018). The projected end points of segmented white matter tracts were visualized using pysurfer (https://pysurfer.github.io). Parameter inputs for the density volume creation function include the maximum distance threshold and decay function for determining which gray matter voxels are "near" an endpoint. Here a $7 \mathrm{~mm}$ gaussian was used, though other decay options include uniform and immediate (i.e., exact endpoint voxel). The final output of this function is an nii.gz volume for both sets of tract endpoints (one for each terminal end of the tract). We define the endpoint density as the nonzero entries of this volume which corresponds to the output of the endpoint-wise decay computation.

Multi subjects Figures. When creating a multi subject plot, this resultant data structure then has those voxels with less than 0.5 endpoint density dropped to zero (to help reduce noise from the smoothing), which is then summed into the MNI-aligned multi-subject data object. Thus, given that the minimum threshold value for the plot is 0.005 of the maximum value, the minimum value being plotted is either 0.5 worth of endpoint density or this threshold value, whichever is largest. This was done because, in cases where the maximum endpoint density is low, the plot becomes somewhat large and diffuse. Multi subject, multi tract plots employed a $5 \mathrm{~mm}$ smooth in order to be appropriately generous when considering overlap.

Individual subjects Figures. Alternatively, in Figures 10 through 16 (and Supplementary Figure 4) b. and c., as well as Supplementary Figures $\mathbf{5}$ through $\mathbf{8}$, only single subjects are depicted, so no warp is applied, and thus the 0.5 floor is also not applied. Additionally, Pysurfer optionally applies a surface smooth when the data is plotted. In the case of multi subject no such surface smoothing was applied, so as to avoid magnifying noise from the inherently noisy process of multi subject alignment. Single subject data employed a a $5 \mathrm{~mm}$ surface smooth kernel. It's still worth noting though that these single subject plots are still, in a sense, amalgams in that they are the sum of 10 ensemble replications.

Tract visualization and statistics. Tracts were visualized using the Matlab Brain Anatomy toolbox (https://github.com/francopestilli/mba; (Pestilli et al. 2014; Goldstone et al. 2015)). This suite of functions takes as its inputs a T1 image and a set of streamlines representing a fiber tract or several fiber tracts and plots them alongside chosen slices of the T1 image in the appropriate spatial orientation. These same streamlines were also used to compute the average length of the fiber. This was achieved by computing the euclidean distance between each sequential node pairing of the fiber. This provided a measure of the length of the streamline, which was itself averaged along all ten replications. This measure was then averaged across all streamlines in a given fiber tract to determine the average fiber length of the fiber tract. In order to determine the volume 
occupied by a given fiber tract we computed the number of nodes per voxel for a given fiber group and then totaled the number of voxels that had at least one node occupying it. A simple streamline count was performed by counting the number of streamlines within a segmented fiber tract. All quantitative statistics were performed with respect to only the positively weighted fibers within a given connectome, while, as previously mentioned, subject-wise mean and error statistics were computed across the 10 ensemble replications. 


\section{REFERENCES.}

Ajina S, Pestilli F, Rokem A, et al (2015) Human blindsight is mediated by an intact geniculo-extrastriate pathway. Elife 4.: doi: 10.7554/eLife.08935

Allen B, Spiegel DP, Thompson B, et al (2015) Altered white matter in early visual pathways of humans with amblyopia. Vision Res 114:48-55

Bajada CJ, Lambon Ralph MA, Cloutman LL (2015) Transport for language south of the Sylvian fissure: The routes and history of the main tracts and stations in the ventral language network. Cortex 69:141-151

Bartolomeo P, Thiebaut de Schotten M, Chica AB (2012) Brain networks of visuospatial attention and their disruption in visual neglect. Front Hum Neurosci 6:110

Bartsch AJ, Geletneky K, Jbabdi S (2013) The temporoparietal fiber intersection area and wernicke perpendicular fasciculus. Neurosurgery 73:E381-2

Basser PJ, Jones DK (2002) Diffusion-tensor MRI: theory, experimental design and data analysis - a technical review. NMR Biomed 15:456-467

Basser PJ, Pajevic S, Pierpaoli C, et al (2000) In vivo fiber tractography using DT-MRI data. Magn Reson Med 44:625-632

Behrens TEJ, Woolrich MW, Jenkinson M, et al (2003) Characterization and propagation of uncertainty in diffusion-weighted MR imaging. Magn Reson Med 50:1077-1088

Broca P (1865) Sur le siège de la faculté du langage articulé. Bull Mem Soc Anthropol Paris 6:377-393

Budisavljevic S, Dell'Acqua F, Castiello U (2018) Cross-talk connections underlying dorsal and ventral stream integration during hand actions. Cortex. doi: 10.1016/j.cortex.2018.02.016

Bullmore E, Sporns O (2009) Complex brain networks: graph theoretical analysis of structural and functional systems. Nat Rev Neurosci 10:186-198

Bullock D, Takemura H, Caiafa CF, et al (2019) Posterior White Matter Data Derivatives. Associative white matter connecting the dorsal and ventral posterior human cortex

Caiafa CF, Pestilli F (2017) Multidimensional encoding of brain connectomes

Catani M, Bodi I, Dell'Acqua F (2012) Comment on "The geometric structure of the brain fiber pathways." Science $337: 1605$

Catani M, de Schotten MT (2012) Atlas of Human Brain Connections (all tracts). In: Atlas of Human Brain Connections. pp 75-238

Catani M, Ffytche DH (2005) The rises and falls of disconnection syndromes. Brain 128:2224-2239

Catani M, Howard RJ, Pajevic S, Jones DK (2002) Virtual in vivo interactive dissection of white matter fasciculi in the human brain. Neuroimage 17:77-94

Catani M, Jones DK, Ffytche DH (2005) Perisylvian language networks of the human brain. Ann Neurol 57:8-16

Catani M, Mesulam M (2008) The arcuate fasciculus and the disconnection theme in language and aphasia: history and current state. Cortex 44:953-961

Catani M, Mesulam MM, Jakobsen E, et al (2013) A novel frontal pathway underlies verbal fluency in primary progressive aphasia. Brain 136:2619-2628

Catani M, Thiebaut de Schotten M (2008) A diffusion tensor imaging tractography atlas for virtual in vivo dissections. Cortex 44:1105-1132

Charcot JM (2016) Lectures on the Localisation of Cerebral and Spinal Diseases, Tr. and Ed. by W.B. Hadden. Palala Press 
Curran EJ (1909) A new association fiber tract in the cerebrum with remarks on the fiber tract dissection method of studying the brain. Journal of Comparative Neurology and Psychology 19:645-656

Davis LE (1921) AN ANATOMIC STUDY OF THE INFERIOR LONGITUDINAL FASCICULUS. Arch Neurol Psychiatry $5: 370$

De Benedictis A, Duffau H, Paradiso B, et al (2014) Anatomo-functional study of the temporo-parieto-occipital region: dissection, tractographic and brain mapping evidence from a neurosurgical perspective. $J$ Anat 225:132-151

De Benedictis A, Petit L, Descoteaux M, et al (2016) New insights in the homotopic and heterotopic connectivity of the frontal portion of the human corpus callosum revealed by microdissection and diffusion tractography. Hum Brain Mapp 37:4718-4735

Decramer T, Swinnen S, van Loon J, et al (2018) White matter tract anatomy in the rhesus monkey: a fiber dissection study. Brain Struct Funct 223:3681-3688

Dejerine J, Dejerine-Klumpke A (1895) Anatomie des centres nerveux

de Schotten MT, Dell'Acqua F, Forkel SJ, et al (2011) A lateralized brain network for visuospatial attention. Nat Neurosci 14:1245-1246

Descoteaux M, Deriche R, Knösche TR, Anwander A (2009) Deterministic and probabilistic tractography based on complex fibre orientation distributions. IEEE Trans Med Imaging 28:269-286

Destrieux C, Fischl B, Dale A, Halgren E (2010) Automatic parcellation of human cortical gyri and sulci using standard anatomical nomenclature. Neuroimage 53:1-15

Dick AS, Bernal B, Tremblay P (2014) The language connectome: new pathways, new concepts. Neuroscientist 20:453-467

Dick AS, Tremblay P (2012) Beyond the arcuate fasciculus: consensus and controversy in the connectional anatomy of language. Brain 135:3529-3550

Dohmen M, Menzel M, Wiese H, et al (2015) Understanding fiber mixture by simulation in 3D Polarized Light Imaging. Neuroimage 111:464-475

Edinger L (1885) Über den Verlauf der centralen Hirnnervenbahnen mit Demonstrationen von Präparaten. Arch Psychiatr Nervenkr 16:858-859

Edinger $L$ (1893) The significance of the cortex considered in connection with a report upon a dog from which the entire cerebrum had been removed by Prof. Goltz. J Comp Neurol 3:69-77

Fang Y, Wang X, Zhong S, et al (2018) Semantic representation in the white matter pathway. PLoS Biol 16:e2003993

Fernández-Miranda JC, Rhoton AL Jr, Alvarez-Linera J, et al (2008) Three-dimensional microsurgical and tractographic anatomy of the white matter of the human brain. Neurosurgery 62:989-1026; discussion 1026-8

Fields RD (2008a) White matter in learning, cognition and psychiatric disorders. Trends Neurosci 31:361-370

Fields RD (2008b) White Matter Matters. Scientific American 298:54-61

Fischl B (2012) FreeSurfer. Neuroimage 62:774-781

Flourens P (1846) Phrenology examined. The American Journal of the Medical Sciences 11:437

Forkel SJ, Thiebaut de Schotten M, Kawadler JM, et al (2014) The anatomy of fronto-occipital connections from early blunt dissections to contemporary tractography. Cortex 56:73-84

Friederici AD (2011) The Brain Basis of Language Processing: From Structure to Function. Physiological Reviews 91:1357-1392

Gabrieli JDE (2009) Dyslexia: a new synergy between education and cognitive neuroscience. Science 325:280-283

Gall FJ (1810) Anatomie et physiologie du système nerveux en général, et du cerveau en particulier: avec des 
observations sur la possibilité de reconnaître plusieurs dispositions intellectuelles et morales de l'homme et des animaux, par la configuration de leurs têtes

Garyfallidis E, Brett M, Amirbekian B, et al (2014) Dipy, a library for the analysis of diffusion MRI data. Front Neuroinform 8:8

Geschwind N (1965) Disconnexion syndromes in animals and man. I. Brain 88:237-294

Geschwind N (1974) Disconnexion Syndromes in Animals and Man. In: Boston Studies in the Philosophy of Science. pp 105-236

Glasser MF, Sotiropoulos SN, Wilson JA, et al (2013) The minimal preprocessing pipelines for the Human Connectome Project. Neuroimage 80:105-124

Goldstone RL, Pestilli F, Börner K (2015) Self-portraits of the brain: cognitive science, data visualization, and communicating brain structure and function. Trends Cogn Sci 19:462-474

Gomez J, Pestilli F, Witthoft N, et al (2015) Functionally defined white matter reveals segregated pathways in human ventral temporal cortex associated with category-specific processing. Neuron 85:216-227

Goodale MA, Milner AD (1992) Separate visual pathways for perception and action. Trends Neurosci 15:20-25

Goryainov SA, Kondrashov AV, Gol'dberg MF, et al (2017) [Long association tracts of the human white matter: an analysis of 18 hemisphere dissections and in vivo HARDI-CSD tractography]. Zh Vopr Neirokhir Im N N Burdenko 81:13-25

Gray H (1918) Anatomy of the Human Body

Grill-Spector K, Kushnir T, Edelman S, et al (1998) Cue-invariant activation in object-related areas of the human occipital lobe. Neuron 21:191-202

Hagmann P, Cammoun L, Gigandet X, et al (2008) Mapping the Structural Core of Human Cerebral Cortex. PLoS Biol 6:e159

Hagmann P, Jonasson L, Maeder P, et al (2006) Understanding Diffusion MR Imaging Techniques: From Scalar Diffusion-weighted Imaging to Diffusion Tensor Imaging and Beyond. Radiographics 26:S205-S223

Hart J (2015) White Matter and Cognition. The Neurobiology of Cognition and Behavior 169-186

Hau J, Sarubbo S, Houde JC, et al (2017) Revisiting the human uncinate fasciculus, its subcomponents and asymmetries with stem-based tractography and microdissection validation. Brain Struct Funct 222:1645-1662

Haxby JV, Hoffman EA, Ida Gobbini M (2000) The distributed human neural system for face perception. Trends in Cognitive Sciences 4:223-233

Homola GA, Jbabdi S, Beckmann CF, Bartsch AJ (2012) A brain network processing the age of faces. PLoS One 7:e49451

Honey CJ, Sporns O (2008) Dynamical consequences of lesions in cortical networks. Hum Brain Mapp 29:802-809

Hubel DH, Livingstone MS (1987) Segregation of form, color, and stereopsis in primate area 18. J Neurosci 7:3378-3415

Jbabdi S, Johansen-Berg H (2011) Tractography: where do we go from here? Brain Connect 1:169-183

Jbabdi S, Sotiropoulos SN, Haber SN, et al (2015) Measuring macroscopic brain connections in vivo. Nat Neurosci 18:1546-1555

Kamali A, Flanders AE, Brody J, et al (2014a) Tracing superior longitudinal fasciculus connectivity in the human brain using high resolution diffusion tensor tractography. Brain Struct Funct 219:269-281

Kamali A, Sair HI, Radmanesh A, Hasan KM (2014b) Decoding the superior parietal lobule connections of the superior longitudinal fasciculus/arcuate fasciculus in the human brain. Neuroscience 277:577-583

Kanwisher N (2010) Functional specificity in the human brain: A window into the functional architecture of the mind. 
Proceedings of the National Academy of Sciences 107:11163-11170

Lawes INC, Barrick TR, Murugam V, et al (2008) Atlas-based segmentation of white matter tracts of the human brain using diffusion tensor tractography and comparison with classical dissection. Neuroimage 39:62-79

Lazar M, Weinstein DM, Tsuruda JS, et al (2003) White matter tractography using diffusion tensor deflection. Hum Brain Mapp 18:306-321

Lee Masson H, Wallraven C, Petit L (2017) "Can touch this": Cross-modal shape categorization performance is associated with microstructural characteristics of white matter association pathways. Hum Brain Mapp 38:842-854

Leong JK, Pestilli F, Wu CC, et al (2016) White-Matter Tract Connecting Anterior Insula to Nucleus Accumbens Correlates with Reduced Preference for Positively Skewed Gambles. Neuron 89:63-69

Libero LE, Burge WK, Deshpande HD, et al (2016) White Matter Diffusion of Major Fiber Tracts Implicated in Autism Spectrum Disorder. Brain Connect 6:691-699

Lunven M, Thiebaut De Schotten M, Bourlon C, et al (2015) White matter lesional predictors of chronic visual neglect: a longitudinal study. Brain 138:746-760

Maier-Hein KH, Neher PF, Houde J-C, et al (2017) The challenge of mapping the human connectome based on diffusion tractography. Nat Commun 8:1349

Mai JK, Majtanik M, Paxinos G (2015) Atlas of the Human Brain. Academic Press

Makris N, Kennedy DN, Mclnerney S, et al (2004) Segmentation of Subcomponents within the Superior Longitudinal Fascicle in Humans: A Quantitative, In Vivo, DT-MRI Study. Cereb Cortex 15:854-869

Makris N, Papadimitriou GM, Kaiser JR, et al (2009) Delineation of the middle longitudinal fascicle in humans: a quantitative, in vivo, DT-MRI study. Cereb Cortex 19:777-785

Makris N, Preti MG, Asami T, et al (2013a) Human middle longitudinal fascicle: variations in patterns of anatomical connections. Brain Struct Funct 218:951-968

Makris N, Preti MG, Wassermann D, et al (2013b) Human middle longitudinal fascicle: segregation and behavioral-clinical implications of two distinct fiber connections linking temporal pole and superior temporal gyrus with the angular gyrus or superior parietal lobule using multi-tensor tractography. Brain Imaging Behav 7:335-352

Makris N, Zhu A, Papadimitriou GM, et al (2017) Mapping temporo-parietal and temporo-occipital cortico-cortical connections of the human middle longitudinal fascicle in subject-specific, probabilistic, and stereotaxic Talairach spaces. Brain Imaging Behav 11:1258-1277

Maldonado IL, de Champfleur NM, Velut S, et al (2013) Evidence of a middle longitudinal fasciculus in the human brain from fiber dissection. J Anat 223:38-45

Martino J, Brogna C, Robles SG, et al (2010a) Anatomic dissection of the inferior fronto-occipital fasciculus revisited in the lights of brain stimulation data. Cortex 46:691-699

Martino J, da Silva-Freitas R, Caballero H, et al (2013a) Fiber dissection and diffusion tensor imaging tractography study of the temporoparietal fiber intersection area. Neurosurgery 72:87-97; discussion 97-8

Martino J, De Lucas EM (2014) Subcortical anatomy of the lateral association fascicles of the brain: A review. Clinical Anatomy 27:563-569

Martino J, De Witt Hamer PC, Berger MS, et al (2013b) Analysis of the subcomponents and cortical terminations of the perisylvian superior longitudinal fasciculus: a fiber dissection and DTI tractography study. Brain Struct Funct 218:105-121

Martino J, García-Porrero JA (2013) Wernicke Perpendicular Fasciculus and Vertical Portion of the Superior Longitudinal Fasciculus. Neurosurgery 73:E382-E383

Martino J, Vergani F, Robles SG, Duffau H (2010b) New insights into the anatomic dissection of the temporal stem 
with special emphasis on the inferior fronto-occipital fasciculus: implications in surgical approach to left mesiotemporal and temporoinsular structures. Neurosurgery 66:4-12

Menjot de Champfleur N, Lima Maldonado I, Moritz-Gasser S, et al (2013) Middle longitudinal fasciculus delineation within language pathways: a diffusion tensor imaging study in human. Eur J Radiol 82:151-157

Miller BL (2010) A Commentary on “Disconnexion Syndromes in Animals and Man.” Neuropsychol Rev 20:126-127

Milner AD, Goodale MA (2008) Two visual systems re-viewed. Neuropsychologia 46:774-785

Mori S, Crain BJ, Chacko VP, van Zijl PC (1999) Three-dimensional tracking of axonal projections in the brain by magnetic resonance imaging. Ann Neurol 45:265-269

Mori S, Kaufmann WE, Davatzikos C, et al (2002) Imaging cortical association tracts in the human brain using diffusion-tensor-based axonal tracking. Magn Reson Med 47:215-223

Mori S, Wakana S, van Zijl PCM, Nagae-Poetscher LM (2005) MRI Atlas of Human White Matter. Elsevier

Mori S, Zhang J (2006) Principles of Diffusion Tensor Imaging and Its Applications to Basic Neuroscience Research. Neuron 51:527-539

Obersteiner H (1890) The Anatomy of the Central Nervous Organs in Health and Disease

Obersteiner H (1889) Anleitung beim Studium des Baues der nervosen Centralorgane im gesunden und kranken Zustande. Am J Psychol 2:304

Ogawa S, Takemura H, Horiguchi $\mathrm{H}$, et al (2014) White matter consequences of retinal receptor and ganglion cell damage. Invest Ophthalmol Vis Sci 55:6976-6986

Panesar SS, Belo JTA, Yeh F-C, Fernandez-Miranda JC (2019) Structure, asymmetry, and connectivity of the human temporo-parietal aslant and vertical occipital fasciculi. Brain Structure and Function 224:907-923

Pestilli F (2018) Human white matter and knowledge representation. PLoS Biol 16:e2005758

Pestilli F, Yeatman JD, Rokem A, et al (2014) Evaluation and statistical inference for human connectomes. Nat Methods 11:1058-1063

Petrides M, Pandya DN (2009) Distinct Parietal and Temporal Pathways to the Homologues of Broca's Area in the Monkey. PLoS Biol 7:e1000170

Ptak R, Schnider A (2010) The dorsal attention network mediates orienting toward behaviorally relevant stimuli in spatial neglect. J Neurosci 30:12557-12565

Reese TG, Heid O, Weisskoff RM, Wedeen VJ (2003) Reduction of eddy-current-induced distortion in diffusion MRI using a twice-refocused spin echo. Magn Reson Med 49:177-182

Reveley C, Seth AK, Pierpaoli C, et al (2015) Superficial white matter fiber systems impede detection of long-range cortical connections in diffusion MR tractography. Proc Natl Acad Sci U S A 112:E2820-8

Rokem A, Takemura H, Bock AS, et al (2017) The visual white matter: The application of diffusion MRI and fiber tractography to vision science. J Vis 17:4

Rokem A, Yeatman JD, Pestilli F, et al (2015) Evaluating the accuracy of diffusion MRI models in white matter. PLoS One 10:e0123272

Rubner, Y., Tomasi, C. \& Guibas, L.J. (2000) The Earth Mover's Distance as a Metric for Image Retrieval. Int J Comput Vis 40:99-121

Saber GT, Pestilli F, Curtis CE (2015) Saccade planning evokes topographically specific activity in the dorsal and ventral streams. J Neurosci 35:245-252

Sakata H, Taira M, Kusunoki M, et al (1997) The TINS Lecture. The parietal association cortex in depth perception and visual control of hand action. Trends Neurosci 20:350-357

Sani I, McPherson BC, Stemmann H, et al (2019) Functionally defined white matter of the macaque monkey brain 
reveals a dorso-ventral attention network. Elife 8.: doi: 10.7554/eLife.40520

Sarubbo S, De Benedictis A, Maldonado IL, et al (2013) Frontal terminations for the inferior fronto-occipital fascicle: anatomical dissection, DTI study and functional considerations on a multi-component bundle. Brain Struct Funct 218:21-37

Sarubbo S, De Benedictis A, Merler S, et al (2016) Structural and functional integration between dorsal and ventral language streams as revealed by blunt dissection and direct electrical stimulation. Hum Brain Mapp $37: 3858-3872$

Schmahmann JD, Pandya DN (2006) Fiber Pathways of the Brain

Schmahmann JD, Pandya DN (2007) Cerebral white matter--historical evolution of facts and notions concerning the organization of the fiber pathways of the brain. J Hist Neurosci 16:237-267

Schmahmann JD, Pandya DN, Wang R, et al (2007) Association fibre pathways of the brain: parallel observations from diffusion spectrum imaging and autoradiography. Brain 130:630-653

Scholz J, Klein M, Behrens T, Johansen-Berg H (2009) White Matter Microstructure Changes In Response to Training. Neurolmage 47:S77

Sotiropoulos SN, Jbabdi S, Xu J, et al (2013) Advances in diffusion MRI acquisition and processing in the Human Connectome Project. Neuroimage 80:125-143

Steinmetz NA, Moore T (2014) Eye movement preparation modulates neuronal responses in area V4 when dissociated from attentional demands. Neuron 83:496-506

Stricker S (1871) Handbuch der Lehre von den Geweben des Menschen und der Thiere

Takemura H, Caiafa CF, Wandell BA, Pestilli F (2016a) Ensemble Tractography. PLoS Comput Biol 12:e1004692

Takemura H, Pestilli F, Weiner KS, et al (2017) Occipital White Matter Tracts in Human and Macaque. Cereb Cortex. doi: 10.1093/cercor/bhx070

Takemura H, Pestilli F, Weiner KS (2018) Comparative neuroanatomy: Integrating classic and modern methods to understand association fibers connecting dorsal and ventral visual cortex. Neurosci Res. doi: 10.1016/j.neures.2018.10.011

Takemura H, Rokem A, Winawer J, et al (2016b) A Major Human White Matter Pathway Between Dorsal and Ventral Visual Cortex. Cerebral Cortex 26:2205-2214

Thomas C, Ye FQ, Irfanoglu MO, et al (2014) Anatomical accuracy of brain connections derived from diffusion MRI tractography is inherently limited. Proc Natl Acad Sci U S A 111:16574-16579

Thomason ME, Thompson PM (2011) Diffusion Imaging, White Matter, and Psychopathology. Annu Rev Clin Psychol 7:63-85

Thompson PM, Stein JL, Medland SE, et al (2014) The ENIGMA Consortium: large-scale collaborative analyses of neuroimaging and genetic data. Brain Imaging Behav 8:153-182

Tournier J-D, Calamante F, Connelly A (2012) MRtrix: Diffusion tractography in crossing fiber regions. Int J Imaging Syst Technol 22:53-66

Tournier J-D, Calamante F, Connelly A (2007) Robust determination of the fibre orientation distribution in diffusion MRI: Non-negativity constrained super-resolved spherical deconvolution. Neuroimage 35:1459-1472

Turken AU, Dronkers NF (2011) The neural architecture of the language comprehension network: converging evidence from lesion and connectivity analyses. Front Syst Neurosci 5:1

Uesaki M, Takemura $\mathrm{H}$, Ashida $\mathrm{H}$ (2018) Computational neuroanatomy of human stratum proprium of interparietal sulcus. Brain Struct Funct 223:489-507

Ungerleider L, Haxby J (1994) "What" and "where" in the human brain. Curr Opin Neurobiol 4:157-165 
Van Essen DC, Ugurbil K, Auerbach E, et al (2012) The Human Connectome Project: a data acquisition perspective. Neuroimage 62:2222-2231

Ventura-Antunes L, Mota B, Herculano-Houzel S (2013) Different scaling of white matter volume, cortical connectivity, and gyrification across rodent and primate brains. Front Neuroanat 7:3

Wakana S, Caprihan A, Panzenboeck MM, et al (2007) Reproducibility of quantitative tractography methods applied to cerebral white matter. Neuroimage 36:630-644

Wandell BA (2016) Clarifying Human White Matter. Annu Rev Neurosci 39:103-128

Wang X, Pathak S, Stefaneanu L, et al (2016) Subcomponents and connectivity of the superior longitudinal fasciculus in the human brain. Brain Struct Funct 221:2075-2092

Wang Y, Fernández-Miranda JC, Verstynen T, et al (2013) Rethinking the role of the middle longitudinal fascicle in language and auditory pathways. Cereb Cortex 23:2347-2356

Wassermann D, Makris N, Rathi Y, et al (2016) The white matter query language: a novel approach for describing human white matter anatomy. Brain Structure and Function 221:4705-4721

Wassermann D, Makris N, Rathi Y, et al (2013) On describing human white matter anatomy: the white matter query language. Med Image Comput Comput Assist Interv 16:647-654

Wasserthal J, Neher P, Maier-Hein KH (2018) TractSeg - Fast and accurate white matter tract segmentation. Neuroimage 183:239-253

Wedeen VJ, Rosene DL, Wang R, et al (2012) The geometric structure of the brain fiber pathways. Science 335:1628-1634

Weiner KS, Yeatman JD, Wandell BA (2016) The posterior arcuate fasciculus and the vertical occipital fasciculus. Cortex. doi: 10.1016/j.cortex.2016.03.012

Wernicke C (1881) Lehrbuch der Gehirnkrankheiten für Aerzte und Studirende

Wernicke C (1874) Der aphasische Symptomencomplex: eine psychologische Studie auf anatomischer Basis

Wu Y, Sun D, Wang Y, et al (2016) Tracing short connections of the temporo-parieto-occipital region in the human brain using diffusion spectrum imaging and fiber dissection. Brain Res 1646:152-159

Yeatman JD, Dougherty RF, Myall NJ, et al (2012) Tract profiles of white matter properties: automating fiber-tract quantification. PLoS One 7:e49790

Yeatman JD, Dougherty RF, Rykhlevskaia E, et al (2011) Anatomical properties of the arcuate fasciculus predict phonological and reading skills in children. J Cogn Neurosci 23:3304-3317

Yeatman JD, Rauschecker AM, Wandell BA (2013) Anatomy of the visual word form area: adjacent cortical circuits and long-range white matter connections. Brain Lang 125:146-155

Yeatman JD, Richie-Halford A, Smith JK, et al (2018) A browser-based tool for visualization and analysis of diffusion MRI data. Nat Commun 9:940

Yeatman JD, Weiner KS, Pestilli F, et al (2014) The vertical occipital fasciculus: a century of controversy resolved by in vivo measurements. Proc Natl Acad Sci U S A 111:E5214-23

Yeh F-C, Panesar S, Fernandes D, et al (2018) Population-averaged atlas of the macroscale human structural connectome and its network topology. Neurolmage 178:57-68

Zhang K, Sejnowski TJ (2000) A universal scaling law between gray matter and white matter of cerebral cortex. Proc Natl Acad Sci U S A 97:5621-5626

Zola-Morgan S (1995) Localization of Brain Function: The Legacy of Franz Joseph Gall (1758-1828). Annual Review of Neuroscience 18:359-383 


\title{
SUPPLEMENTARY MATERIAL
}

\section{Associative white matter connecting the dorsal and ventral posterior human cortex}

\author{
Bullock, D., Takemura, H., Caiafa, C. F., Kitchell, L., McPherson, B., Caron, B., and Pestilli, F.
}

\section{Affiliation}

1. Daniel Bullock. Department of Psychological and Brain Sciences, Program in Neuroscience, Indiana University Bloomington, 1101 E 10th Street, Bloomington, Indiana USA 47405; Center for Information and Neural Networks (CiNet), National Institute of Information and Communications Technology. Email: dnbulloc@indiana.edu

2. Hiromasa Takemura. Center for Information and Neural Networks (CiNet), National Institute of Information and Communication Technology, and Osaka University; Graduate School of Frontier Biosciences, Osaka University, 1-4 Yamadaoka, Suita-shi, Osaka Japan 565-0801. Email: htakemur@nict.go.jp

3. Cesar F. Caiafa. Department of Psychological and Brain Sciences, Indiana University Bloomington, $1101 \mathrm{E} 10$ th Street, Bloomington, Indiana USA 47405. Instituto Argentino de Radioastronomía (CCT-La Plata, CONICET; CICPBA), CC5 V. Elisa, ARGENTINA, 1894. Facultad de Ingeniería - Universidad de Buenos Aires, Buenos Aires, ARGENTINA, C1063ACV. Email: ccaiafa@gmail.com

4. Lindsey Kitchell. Department of Psychological and Brain Sciences, Department of Cognitive Science, Indiana University Bloomington, 1101 E 10th Street, Bloomington, Indiana USA 47405; Email: kitchell@umail.iu.edu

5. Brent McPherson. Department of Psychological and Brain Sciences, Department of Cognitive Science, Indiana University Bloomington, 1101 E 10th Street, Bloomington, Indiana USA 47405; Email: bcmcpher@umail.iu.edu

6. Bradley Caron. Program in Neuroscience, Vision Science, Indiana University Bloomington,702 N Walnut Grove St, Bloomington, Indiana USA 47405; Email: bacaron@iu.edu

7. Franco Pestilli. Department of Psychological and Brain Sciences, Engineering, Computer Science, Programs in Neuroscience and Cognitive Science, School of Optometry, and Indiana Network Science Institute, Indiana University Bloomington, $1101 \mathrm{E}$ 10th Street, Bloomington, Indiana USA 47405. Email: franpest@indiana.edu

Contribution D.N.B. and F.P. conceptualized and performed analyses. H.T. and C.F.C. provided data curation and software. D.N.B., H.T., C.F.C., and F.P. wrote the manuscript. L.K., B.M., and B.C. provided validation and software.

Competing interests The authors declare no competing financial interests.

Corresponding authors Franco Pestilli franpest@indiana.edu

\begin{abstract}
Historically, the primary focus of studies of human white matter tracts has been on large tracts that connect anterior to posterior cortical regions. These include the superior longitudinal fasciculus (SLF), the inferior longitudinal fasciculus (ILF), and the inferior fronto-occipital fasciculus (IFOF). Recently, more refined and well understood tractography methods have facilitated the characterization of several tracts in the posterior of the human brain that connect dorsal to ventral cortical regions. These include the vertical occipital fasciculus (VOF), the posterior arcuate fasciculus (pArc), the temporo-parietal connection (TP-SPL), and the middle longitudinal fasciculus (MdLF). The addition of these dorso-ventral connective tracts to our standard picture of white matter architecture results in a more complicated pattern of white matter connectivity than previously considered. Dorso-ventral connective tracts may play a role in transferring information from superior horizontal tracts, such as the SLF, to inferior horizontal tracts, such as the IFOF and ILF. We present a full anatomical delineation of these major dorso-ventral connective white matter tracts (the VOF, pArc, TP-SPL, MdLF). We show their spatial layout and cortical termination mappings in relation to the more established horizontal tracts (SLF, IFOF, ILF, Arc) and consider standard values for quantitative features associated with the aforementioned tracts. We hope to facilitate further study on these tracts and their relations. To this end, we also share links to automated code that segments these tracts, thereby providing a standard approach to obtaining these tracts for subsequent analysis. We developed open source software to allow reproducible segmentation of the tracts: https://github.com/brainlife/Vertical_Tracts. Finally, we make the segmentation method available as an open cloud service on the data and analyses sharing platform brainlife.io. Investigators will be able to access these services and upload their data to segment these tracts.
\end{abstract}

Keywords: diffusion imaging; white matter; historical; tractography; dorsal and ventral streams; computational neuroanatomy 


\section{Acknowledgements}

This research was supported by NSF IIS-1636893, NSF BCS-1734853, NIH NCATS UL1TR002529, a Microsoft Research Award, Japan Society for the Promotion of Science (JSPS) KAKENHI (JP17H04684, JP15J00412) and the Indiana University Areas of Emergent Research initiative "Learning: Brains, Machines, Children." In part by NIH NCATS UL1TR002529 to F.P.. D.N.B. and B.M. were partially funded via NIH NIMH 5 T32 MH103213 to B. Hetrick and B. D'Onofrio. We thank Soichi Hayashi, Steven O'Riley, David Hunt and Aman Arya for contributing to the development of brainlife.io, Craig Stewart, Winona Snapp-Childs, David Hancok and Jeremy Fischer for support with jetstream-cloud.org (NSF ACl-1445604). Data were provided in part by the Human Connectome Project, WU-Minn Consortium (Principal Investigators: David Van Essen and Kamil Ugurbil; 1U54MH091657) funded by the 16 NIH Institutes and Centers that support the NIH Blueprint for Neuroscience Research; and by the McDonnell Center for Systems Neuroscience at Washington University. Data were provided in part by Brian Wandell (Stanford University; https://purl.stanford.edu/bb060nk0241). Thanks to Sophia Vinci-Booher for comments on early versions of the manuscript. Thanks also to Josh Faskowitz for help with software.

\section{Compliance with ethical standards.}

Conflicts of interest:

Authors declare no conflicts of interest.

Research involving human participants and/or animals:

Data collection was approved by the respective Institutional Review Boards (IRBs) of the University of Washington, Saint Louis (HCP dataset) and Stanford University (STN dataset).

Informed consent.

All participants provided written informed consent to participate in the project 
Despite the limited number of studies two of the tracts of interest have been reported using both imaging modalities (dMRI) and post-mortem dissection in the human brain. This is the case for the pArc (Catani et al. 2004; Lawes et al. 2008; Martino and García-Porrero 2013; Wu et al. 2016) and MdLF-SPL(Lawes et al. 2008; Makris et al. 2013; Wang et al. 2013). Both the TP-SPL (Makris et al. 2013; Kamali et al. 2014a; Wu et al. 2016) and MdLF-Ang (Kamali et al. 2014b; Wu et al. 2016) have instead only been reported using dMRI.

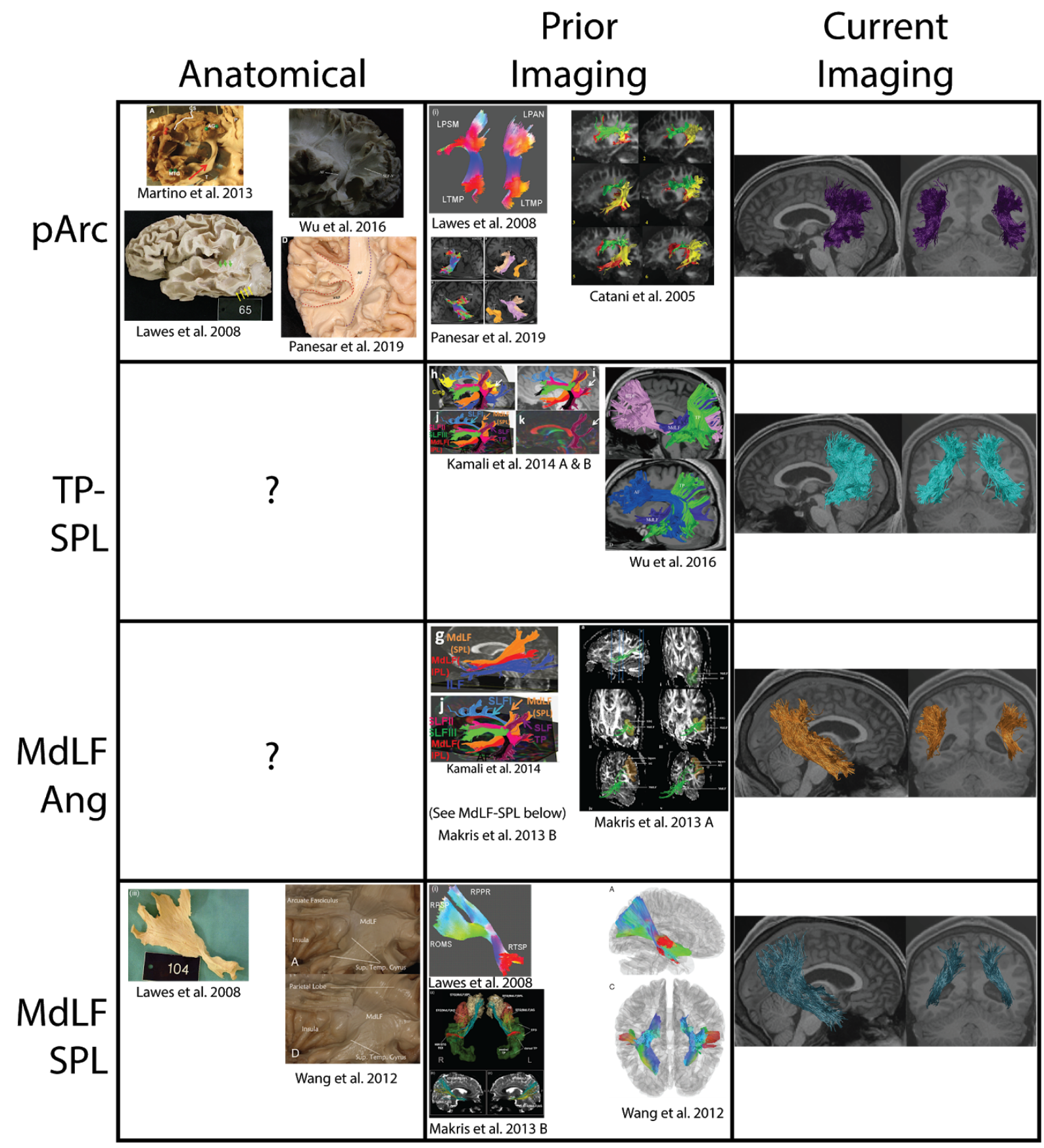

Supplementary Fig. 1 Evidence from different data modalities supporting the four tracts of interest discussed in the main text. Rows correspond to specific tracts while columns correspond to evidential context. In the first two rows (Columns 1 and 2) figures are taken from their respective papers (see captions below each figure). Column 3 presents a tractography segmentation from the current study for comparison. The question 
marks in the "Anatomical" column, found in the TP-SPL and MdLF-Ang rows, indicate the current lack of anatomical evidence for the corresponding tracts

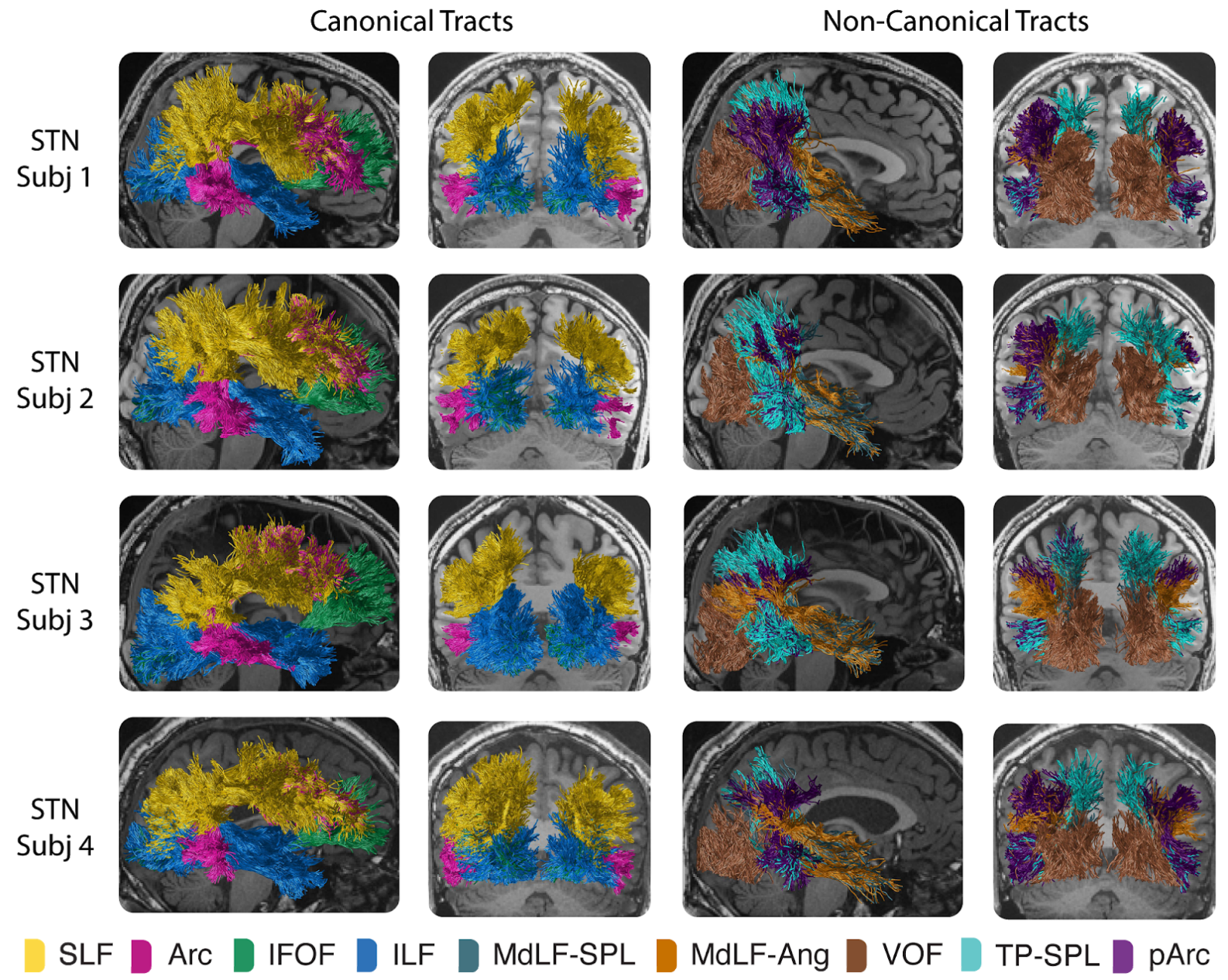

Supplementary Fig. 2 Anatomy of canonical (horizontal) and non-canonical (dorso-ventral connecting) white matter tracts identified from STN dataset. Conventions are identical to those used in Figure 2 


\section{Quantitative characterization of canonical and non-canonical tracts}

\begin{tabular}{|c|c|c|c|c|c|c|c|c|c|c|}
\hline & \multicolumn{2}{|c|}{ Count } & \multicolumn{2}{|c|}{ Length $(\mathrm{mm})$} & \multicolumn{2}{|c|}{ Volume $\left(\mathrm{mm}^{3}\right)$} & \multicolumn{2}{|c|}{ Volume (\%) } & \multicolumn{2}{|c|}{ EMD } \\
\hline IFOF $L$ & 59 & 6.7 & 159.56 & 2.48 & 5943.9 & 576.2 & 0.998 & 0.097 & 7.72E-05 & $2.44 E-05$ \\
\hline $\boldsymbol{R}$ & 36 & 5.3 & 157.60 & 2.51 & 4018.7 & 484.8 & 0.689 & 0.084 & 7.75E-05 & 2.82E-05 \\
\hline Arcuate $L$ & 572.6 & 25.4 & 117.27 & 0.79 & 19135.9 & 564.9 & 3.310 & 0.097 & 1.61E-03 & 2.70E-04 \\
\hline $\boldsymbol{R}$ & 401.4 & 21.0 & 125.69 & 1.15 & 17081.8 & 633.8 & 2.957 & 0.112 & 6.51E-04 & $1.63 E-04$ \\
\hline ILF $L$ & 229.6 & 14.4 & 107.83 & 0.98 & 11555.5 & 471.2 & 2.017 & 0.082 & 3.22E-04 & $1.15 E-04$ \\
\hline $\boldsymbol{R}$ & 236.1 & 11.3 & 106.07 & 0.91 & 12562.9 & 371.5 & 2.176 & 0.064 & 3.14E-04 & $6.04 E-05$ \\
\hline SLF $L$ & 1707.9 & 42.0 & 84.47 & 0.55 & 45106.5 & 714.5 & 7.777 & 0.125 & 1.62E-03 & $2.86 E-04$ \\
\hline$R$ & 2077.7 & 50.1 & 88.30 & 0.41 & 49388.2 & 750.0 & 8.529 & 0.131 & 2.30E-03 & 8.17E-05 \\
\hline MdLF-Ang $L$ & 138.35 & 11.9 & 69.61 & 2.58 & 6253.3 & 550.0 & 1.093 & 0.095 & 1.40E-04 & $4.81 E-05$ \\
\hline $\mathbf{R}$ & 136.97 & 9.1 & 70.14 & 2.46 & 6985.4 & 408.5 & 1.201 & 0.070 & 1.12E-04 & 2.83-E05 \\
\hline MdLF-SPL $L$ & 14.6 & 3.2 & 105.76 & 3.48 & 1378.7 & 298.6 & 0.239 & 0.053 & 2.86E-05 & 8.57E-06 \\
\hline $\boldsymbol{R}$ & 25.9 & 5.5 & 106.32 & 3.67 & 2356.1 & 479.9 & 0.407 & 0.082 & 4.14E-05 & 7.17E-06 \\
\hline pArc $L$ & 257.1 & 16.8 & 62.21 & 0.65 & 8356.3 & 378.4 & 1.463 & 0.066 & 5.00E-04 & $1.31 E-04$ \\
\hline $\boldsymbol{R}$ & 332.6 & 13.8 & 62.32 & 0.54 & 8959.5 & 257.9 & 1.513 & 0.045 & 9.35E-04 & $4.48 E-04$ \\
\hline VOF $L$ & 470.9 & 17.4 & 35.33 & 0.41 & 8676.9 & 198.5 & 1.511 & 0.034 & 1.23E-03 & $2.42 E-04$ \\
\hline$R$ & 404.6 & 17.7 & 34.28 & 0.57 & 8255.1 & 267.6 & 1.429 & 0.045 & 9.39E-04 & 2.01E-04 \\
\hline TP-SPL $L$ & 57.9 & 7.0 & 79.41 & 2.85 & 3570.7 & 414.7 & 0.641 & 0.074 & 7.10E-05 & $2.62 E-05$ \\
\hline$R$ & 102.9 & 8.7 & 75.70 & 1.48 & 5665.3 & 468.1 & 1.003 & 0.084 & 1.41E-04 & $4.82 E-05$ \\
\hline
\end{tabular}

Supplementary Table 1. Quantitative properties of major tracts in HCP 3 T dataset. For each tract (Left, $L$ and Right, $R$ ) we provide the average count (average number of streamlines), average tract length (average length of streamlines composing tract, in millimeters), average volume (average volume occupied by tract, in cubic millimeters), volume proportion (white matter volume occupied by tract relative to whole brain white matter volume, in percentage). Additional statistical metrics are provided are in the form of statistical evidence (scanner units of diffusion accounted for by the tract, as modeled by the earth mover's distance). Each mean measure is reported followed by the standard deviation across subjects, STD values in italics

\begin{tabular}{|c|c|c|c|c|c|c|c|c|c|c|}
\hline \multirow[b]{2}{*}{ IFOF $L$} & \multicolumn{2}{|c|}{ Count } & \multicolumn{2}{|c|}{ Length $(\mathrm{mm})$} & \multicolumn{2}{|c|}{ Volume $\left(\mathrm{mm}^{3}\right)$} & \multicolumn{2}{|c|}{ Volume (\%) } & \multicolumn{2}{|c|}{ EMD } \\
\hline & 28 & 5.1 & 155.09 & 3.05 & 3293.9 & 493.2.2 & 0.561 & 0.085 & 5.79E-05 & 2.20E-05 \\
\hline $\boldsymbol{R}$ & 22 & 4.3 & 153.25 & 3.77 & 2531.5 & 402.1 & 0.434 & 0.068 & 4.38E-05 & $7.84 E-06$ \\
\hline Arcuate $L$ & 365.5 .6 & 16.3 & 108.90 & 0.71 & 14306.6 & 310.7 & 2.485 & 0.054 & $9.18 \mathrm{E}-04$ & $2.42 E-04$ \\
\hline $\boldsymbol{R}$ & 261.4 & 13.9 & 110.24 & 1.02 & 11680.8 & 374.4 & 2.056 & 0.064 & 8.35E-04 & 3.30E-04 \\
\hline ILF $L$ & 156.1 & 9.3 & 102.04 & 1.48 & 8681.1 & 401.8 & 1.480 & 0.070 & 1.80E-04 & $9.58 E-05$ \\
\hline $\boldsymbol{R}$ & 131.9 & 10.6 & 96.84 & 1.08 & 8169.8 & 442.1 & 1.392 & 0.077 & 1.23E-04 & 2.87E-05 \\
\hline SLF $L$ & 1171.5 & 36.5 & 72.65 & 0.52 & 32136.9 & 672.9 & 5.505 & 0.117 & 1.27E-03 & 1.17E-04 \\
\hline $\boldsymbol{R}$ & 928.2 & 20.8 & 77.63 & 0.59 & 26896.6 & 512.2 & 4.628 & 0.090 & 1.29E-03 & 3.07E-04 \\
\hline MdLF-Ang $L$ & 56.3 & 8.0 & 79.08 & 3.05 & 3617.3 & 443.7 & 6.289 & 0.077 & 4.69E-05 & $1.27 E-05$ \\
\hline $\mathbf{R}$ & 40.9 & 6.3 & 56 & 4.78 & 194 & 325.8 & 3.356 & 0.055 & 5.85E-05 & $1.29 E-05$ \\
\hline MdLF-SPL $L$ & 18.1 & 3.3 & 107.76 & 4.58 & 7 & 5.1 & 81 & 0.051 & 2.61E-05 & $7.62 E-06$ \\
\hline$R$ & 12.0 & 2.6 & 9 & 7.14 & 3 & 233.8 & 84 & 41 & -05 & $5.52 E-06$ \\
\hline pArc $L$ & 169.9 & 11.3 & 65.79 & 0.68 & 2 & 347.8 & 1.103 & 0.059 & 3.06E-04 & 1.67E-04 \\
\hline$R$ & 51.0 & 7.1 & 66.19 & 1.84 & 2514.1 & 336.6 & 0.440 & 0.059 & 1.00E-04 & $7.23 E-05$ \\
\hline VOF $L$ & 273.2 & 12.6 & 31.36 & 0.57 & 5510.0 & 223.9 & 0.970 & 0.038 & $6.57 \mathrm{E}-04$ & $4.76 E-04$ \\
\hline$R$ & 253.7 & 11.4 & 30.74 & 0.59 & 4928.4 & 203.7 & 0.870 & 0.036 & 8.84E-04 & 4.17E-04 \\
\hline TP-SPL $L$ & 37.0 & 5.1 & 80.52 & 1.92 & 2505.4 & 325.5 & 0.439 & 0.057 & 4.95E-05 & $1.43 E-05$ \\
\hline$R$ & 44.5 & 4.6 & 77.01 & 2.32 & 2485.3 & 267.1 & 0.449 & 0.047 & 5.90E-05 & 4.03E-05 \\
\hline
\end{tabular}

Supplementary Table 2. Quantitative properties of major tracts in STN96 dataset. Conventions are identical to those in Supplementary Table 1 
Volume measures were computed by identifying and counting those $1 \mathrm{~mm}$ volumes that contained at least one streamline node from the TOI. The SLF stands out as occupying the most volume (approximately 45,000 and $50,000 \mathrm{~mm}^{3}$ in the left and right hemispheres, respectively), easily doubling the next closest tract, the Arcuate $\left(19,000\right.$ and $17,000 \mathrm{~mm}^{3}$ in the left and right hemispheres, respectively). The remaining tracts, both canonical and non canonical, range between 4000 and 11,0000 cubic millimeters. Supplementary Tables 1 and 2 also reveal a number of significant asymmetries in volume, for example in the IFOF, the MdLF-SPL and the TP-SPL. Volume proportion serves as another means of considering the volumetric properties of white matter tracts, but has the added benefit of controlling for cross subject variation in brain volume.

Statistical measures using diffusion-derived properties provide another means of characterizing and standardizing these tracts (Pestilli et al. 2014; Gomez et al. 2015; Pestilli 2015; Leong et al. 2016; Takemura et al. 2016; Caiafa and Pestilli 2017; Rokem et al. 2017). The statistical evidence corresponds to the amount of model error (relative to the whole brain tractography) accounted for by the tract. Here, in the evidence column of Supplementary Table 1 , we see the evidence associated with the aforementioned tracts. As with count (and volume), the SLF and Arcuate are noted to exhibit the greatest quantities of evidence, though the VOF and pArc are also observed to exhibit moderate levels of evidence as well. Supplementary Tables 1 and 2 reports average streamline count, tract length, volume, white matter proportion, and statistical evidence (EMD) across subjects for the HCP 3T dataset (Supplementary table 1 reports the same measures for the STN96 dataset). The non-canonical tracts, aside from the MdLF, are measured to be significantly shorter than the shortest of the canonical tracts (i.e., the SLF with an average of $86 \mathrm{~mm}$ ). The MdLF (mean $110 \mathrm{~mm}$ ) is found to be longer than either the SLF (mean $86 \mathrm{~mm}$ ) or the ILF (mean $95 \mathrm{~mm}$ ), but shorter than the Arc (mean $121 \mathrm{~mm}$ ). As expected, the IFOF is found to be notably longer than any of the other canonical tracts $(159 \mathrm{~mm}$ versus $121 \mathrm{~mm}$ for the next longest tract, the Arc). In order to illustrate the wide range of tract lengths measured across canonical and non-canonical tracts we can note that the VOF, the shortest tract, is on average $35 \mathrm{~mm}$ as compared to the IFOF, the longest tract, which is $157 \mathrm{~mm}$, on average, indicating a range of $122 \mathrm{~mm}$ in the tracts described here. Conversely, the MdLF (the longest dorso-ventral associative tract) is noted to be $110 \mathrm{~mm}$ while the SLF (the shortest canonical tract) is noted to be $86 \mathrm{~mm}$, a difference of $24 \mathrm{~mm}$ in favor of the non-canonical tract. In light of this, we see that length is not necessarily a unifying or distinguishing characteristic of the canonical and non-canonical connective tracts.
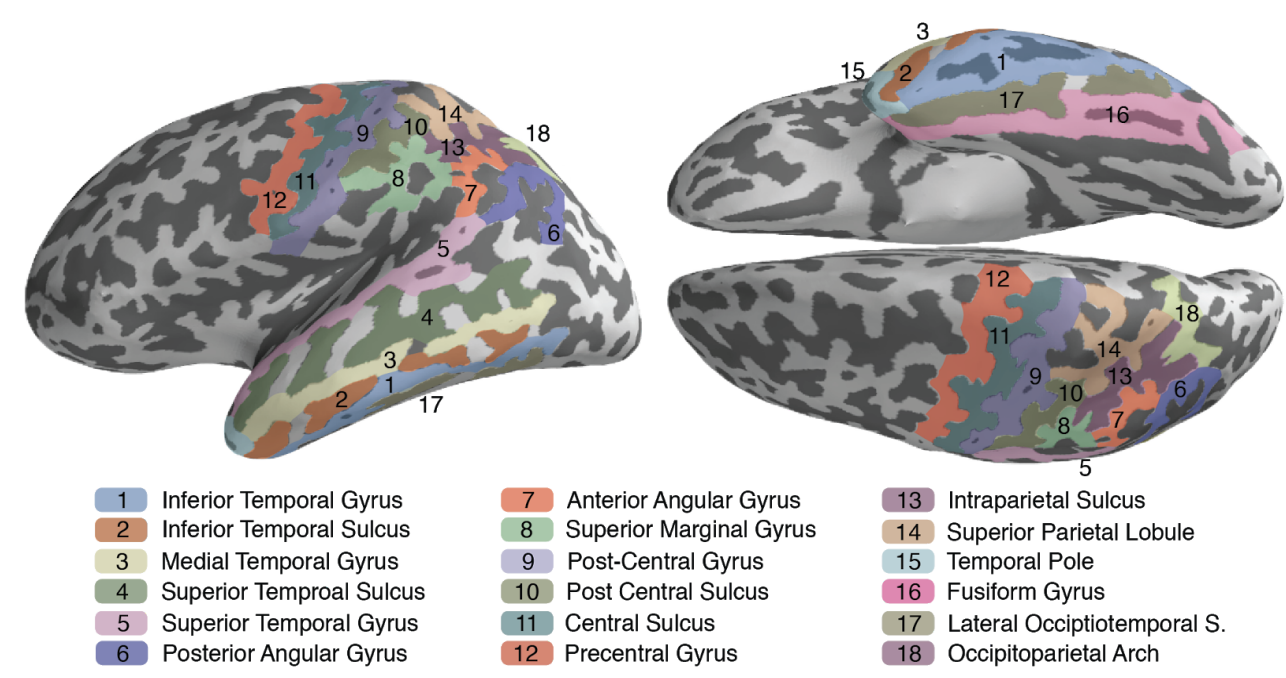

13 Intraparietal Sulcus

14 Superior Parietal Lobule

15 Temporal Pole

16 Fusiform Gyrus

17 Lateral Occiptiotemporal S

18 Occipitoparietal Arch

Supplementary Fig. 3 Summary of major organization of relevant cortical gyri and sulci and white matter tracts. A sagittal view of a inflated cortical surface. Labeling has been applied to identify regions that are relevant to the posterior or superior terminations of relevant fiber tracts 
Supplementary Figure 4 reports the overlap in cortical termination between the SLF, pArc and TP-SPL (Catani et al. 2004; de Schotten et al. 2011; Catani and de Schotten 2012; Martino et al. 2013). Panels B and $\mathrm{C}$ show that the comparatively large SLF shares termination regions with both the TP-SPL

(Supplementary Figure $\mathbf{4 b}$ and $4 \mathrm{~d}$, green) and pArc (Supplementary Figure 5C, orange). Interestingly, the terminations of the SLF appear to extend slightly more posterior than the terminations of either tract. Also, we see that the SLF contains terminations which extend somewhat inferior of the pArc, into the posterior superior temporal gyrus (Supplementary Figure 3, area 5).

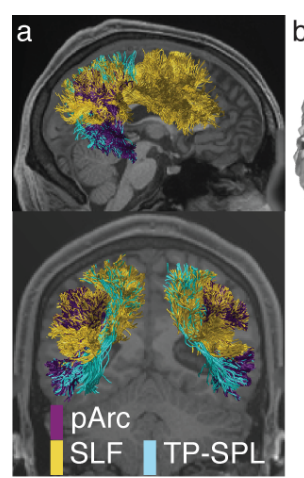

d

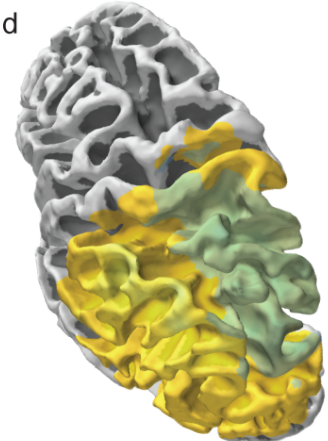

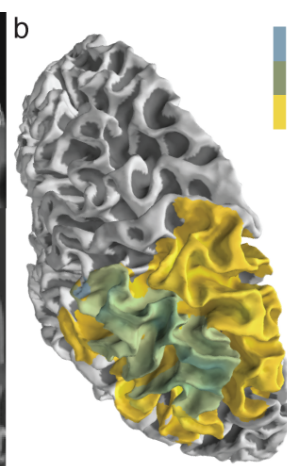

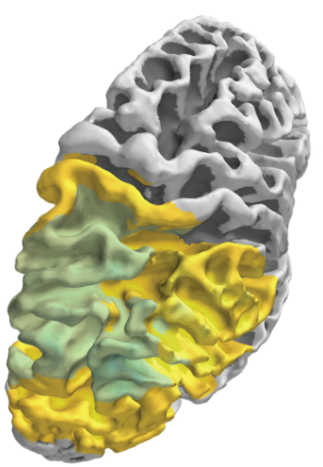

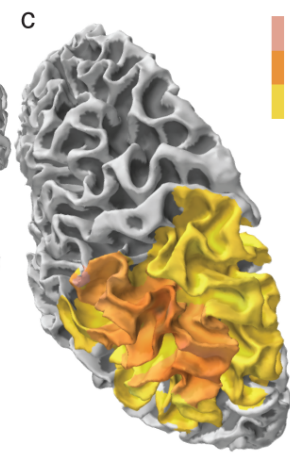
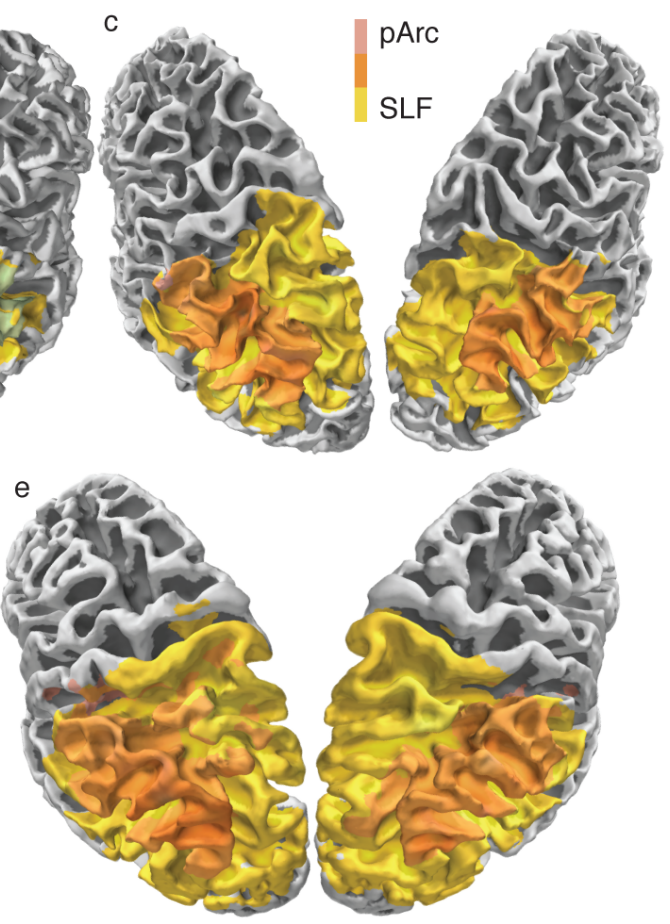

Supplementary Fig. 4 Relation between the SLF, pArc and TP-SPL cortical termination patterns. a. Three tracts anatomy, SLF, pArc and TP-SPL. Anatomy of the three tracts of interest plotted over a representative sagittal and coronal brain slice for a single subject (HCP 105115). b. Cortical endpoint density mapping SLF and TP-SPL. Density projections plotted on both cortical hemispheres. Blue coloring corresponds to nearby TP-SPL endpoints while yellow coloring corresponds to nearby SLF endpoints. Green arises in areas of overlap. c. Cortical endpoint density mapping SLF and pArc. Density projections plotted on both cortical hemispheres. Red coloring corresponds to nearby pArc endpoints while yellow coloring corresponds to nearby SLF endpoints. Orange arises in areas of overlap. $\mathbf{a}$. and $\mathbf{b}$. depict data for a single subject (HCP 105115). d and e. Compound tract endpoint overlap maps across 8 subjects. Maps depict sum plots across subjects displayed on the MNI template (same color conventions as a and $\mathbf{b}$; https://doi.org/10.6084/m9.figshare.4223811.v1). 


\begin{tabular}{|c|c|c|c|c|c|c|c|c|}
\hline \multicolumn{7}{|c|}{ Endpoint inclusion criteria (AND) } & \multirow[b]{2}{*}{$\begin{array}{l}\text { Streamline inclusion criteria } \\
\text { (AND) }\end{array}$} & \multirow[b]{2}{*}{ Streamline exIcusion criteria (NOT) } \\
\hline $\begin{array}{l}\text { Tract } \\
\text { Name }\end{array}$ & $\begin{array}{c}\text { ROI 1 } \\
\text { D2009 } \\
\text { numbers } \\
\text { (11xxx/12xxx) }\end{array}$ & $\begin{array}{c}\text { ROI } 1 \\
\text { English equivalent }\end{array}$ & $\begin{array}{c}\text { ROI } 1 \\
\text { Additional } \\
\text { Criteria }\end{array}$ & $\begin{array}{c}\mathrm{ROI} 2 \\
\mathrm{D} 2009 \\
\text { numbers } \\
(11 \mathbf{x x x} / 12 \times x \mathbf{x})\end{array}$ & $\begin{array}{c}\text { ROI } 2 \\
\text { English equivalent }\end{array}$ & $\begin{array}{c}\text { ROI } 2 \\
\text { Additional } \\
\text { Criteria }\end{array}$ & & \\
\hline pArc & $\begin{array}{l}\text { 157, } 127,168 \\
136,126,125\end{array}$ & $\begin{array}{l}\text { Intraparietal and transverse parietal } \\
\text { sulci; } \\
\text { Superior parietal gyrus; } \\
\text { Postcentral sulcus; } \\
\text { Superior temporal gyrus: planum } \\
\text { temporale; } \\
\text { Inferior parietal gyrus: supermarginal } \\
\text { gyrus; } \\
\text { Inferior parietal gyrus: angular gyrus }\end{array}$ & None & $\begin{array}{l}\text { 121, 161, 137, } \\
162,138,173\end{array}$ & $\begin{array}{l}\text { Temporal: lateral fusiform gyrus; } \\
\text { Lateral temproal sulcus; } \\
\text { Inferior temporal gyrus; } \\
\text { Medial temporal and lingual sulci; } \\
\text { Middle temporal gyrus; } \\
\text { Inferior temporal sulcus; }\end{array}$ & $\begin{array}{l}\text { Poster to } \\
\text { posterior edge of } \\
\text { putamen (ROls } \\
12 / 51)\end{array}$ & $\begin{array}{l}\text { > Passes through transverse } \\
\text { plane at inferior border of } \\
\text { Jensen sulcus (ROI XX156) } \\
\text { > Passes through transverse } \\
\text { plane at inferior border of } \\
\text { planum temporale (ROI XX136) }\end{array}$ & $\begin{array}{l}\text { > No part superior to the inferior border of Jensen } \\
\text { Sulcus (ROI XX156) passes through coronal } \\
\text { plane at the posterior border of the superior } \\
\text { frontal gyrus (ROI XX116) } \\
\text { > DOES NOT pass through the overlap of inflated } \\
\text { (27 mm diameter smoothing kernel) } \\
\text { precuneus (ROI XX127) and intraparietal and } \\
\text { transverse parietal sulci (ROI XX157) } \\
\text { > No part posterior to the posterior border of (ROI } \\
\text { XX162) passes through transverse plane at the } \\
\text { superior border of the medial temporal and } \\
\text { lingual sulci (ROI XX162) }\end{array}$ \\
\hline TPC & See above & See above & None & See above & See above & See above & $\begin{array}{l}\text { > See first criteria above } \\
\text { > See second criteria above } \\
\text { > Passes through the overlap } \\
\text { of inflated (27 mm diameter } \\
\text { smoothing kernel) precuneus } \\
\text { (ROI XX127) and intraparietal } \\
\text { and transverse parietal sulci } \\
\text { (ROI XX157) }\end{array}$ & > See first criteria above \\
\hline $\begin{array}{l}\text { MdLF- } \\
\text { Ang }\end{array}$ & See above & See above & None & $\begin{array}{l}\text { 134, } 144 \\
174,135\end{array}$ & $\begin{array}{l}\text { superior temporal gyrus: lateral } \\
\text { part; } \\
\text { temporal pole; } \\
\text { superior temporal sulcus; } \\
\text { planum polare; }\end{array}$ & $\begin{array}{l}\text { Anterior to } \\
\text { posterior edge of } \\
\text { amygdala (ROls } \\
18 / 54)\end{array}$ & None & $\begin{array}{l}\text { > No part interects with inferior lateral ventricle, } \\
\text { Putamen, Pallidum, Hippocampus, Amygdala } \\
\text { (ROIs } 5 / 44,12 / 51,13 / 52,17 / 53,18 / 54 \text { ) } \\
>\text { No part superior to the superior border of } \\
\text { circular sulcus of the insula (ROI XX156) passes } \\
\text { through coronal plane at the anterior border of } \\
\text { the subparietal sulcus (ROI XX172) } \\
>\text { No midpoints above the highest subcortical ROI } \\
\text { (inferior lateral ventricle, Putamen, Pallidum, } \\
\text { Hippocampus, Amygdala [ROIs } 5 / 44,12 / 51 \text {, } \\
13 / 52,17 / 53,18 / 54] \text { ) between the anterior border } \\
\text { of the subparietal sulcus (ROI XX172) and the } \\
\text { posterior edge of amygdala (ROls } 18 / 54 \text { ). } \\
>\text { No midpoints anterior to the anterior edge of } \\
\text { the amygdala (ROIs } 18 \text { / } 54 \text { ). } \\
>\text { DOES NOT pass through the overlap of } \\
\text { inflated (27 mm diameter smoothing kernel) } \\
\text { precuneus (ROI XX127) and intraparietal and } \\
\text { transverse parietal sulci (ROI XX157). }\end{array}$ \\
\hline $\begin{array}{l}\text { MdLF- } \\
\text { SPL }\end{array}$ & See above & See above & None & See above & See above & See above & $\begin{array}{l}\text { > Passes through the overlap } \\
\text { of inflated ( } 27 \mathrm{~mm} \text { diameter } \\
\text { smoothing kernel) precuneus } \\
\text { (ROI XX127) and intraparietal } \\
\text { and transverse parietal sulci } \\
\text { (ROI XX157) }\end{array}$ & \\
\hline
\end{tabular}

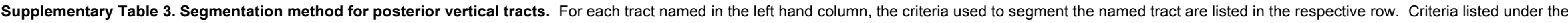

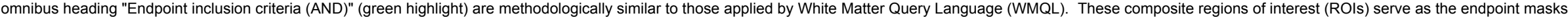
for the given tracks such that streamlines classified as being a member of the given tract must have endpoints in the specified ROls. Criteria under the latter two column headings (blue highlight) are not replicable using WMQL, which accounts for this study's use of an alternative segmentation method (description found in methods section of main text). These criteria relate to NOT planes and borders (areas that no streamline can intersect with) and midpoint criteria (requirements specific to the midpoints of tract streamlines, taken to be akin to the "saddle" of an anatomical white matter tract). ROls correspond to Freesurfer's Destrieux 2009 atlas. 

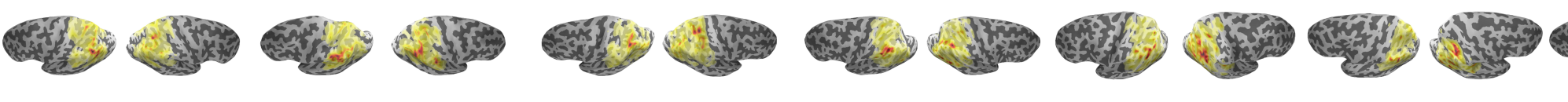

ILF<smiles></smiles>

\section{\%}
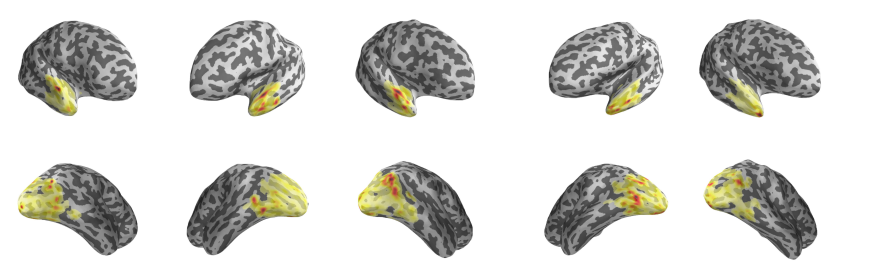

(1)
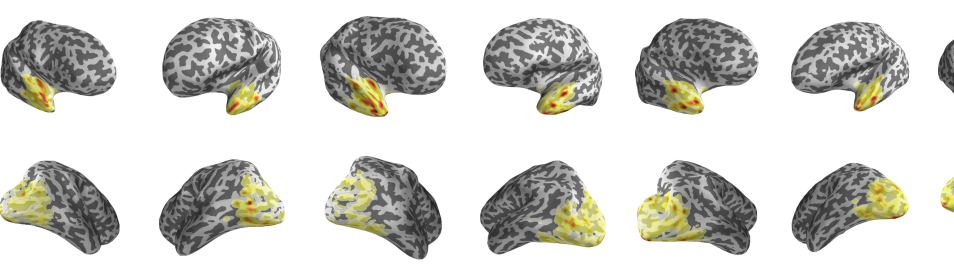

/I
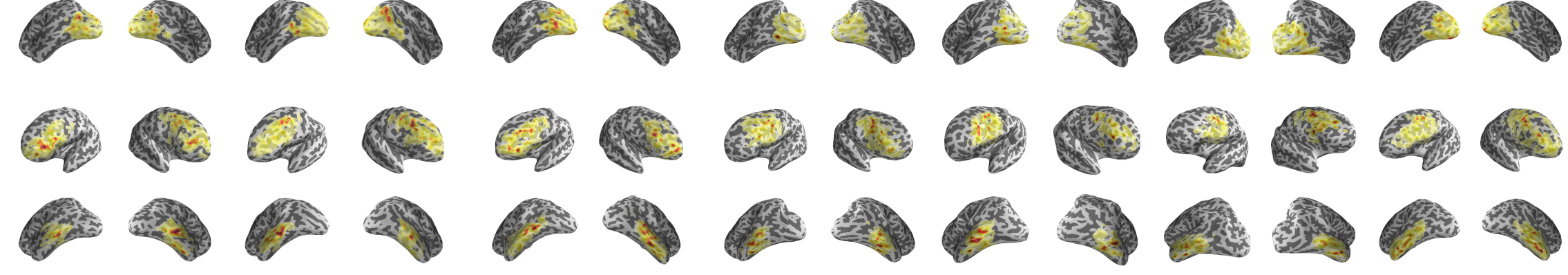

$P /$
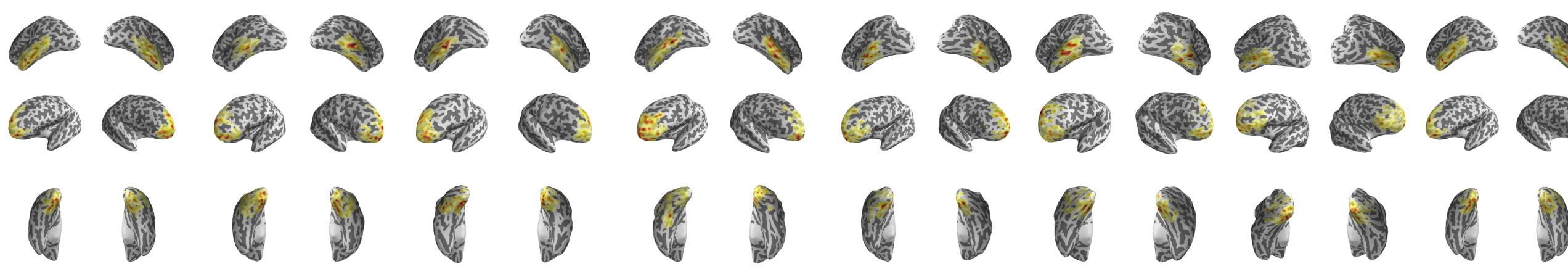

\section{Minimum normalized Density}

Maximum normalized
Density

Supplementary Fig. 5. Cortical termination mappings for cannonical tracts. Horziontal axis labeling corresponds to subject ID (for HCP, subject numbers; for STN96, subject initials), while L/R indicates hemisphere (L=left, $R=$ right). Vertical axis labeling corresponds to tract name, while $\mathrm{A} / \mathrm{S}$ or $\mathrm{P} / \mathrm{l}$ indicate tract termination group (A/S = anterior or superior; $\mathrm{P} / \mathrm{l}=$ posterior or inferior). Heatmaps depicted on cortical surfaces correspond to normalized endpoint density mappings; lighter yellows indicate less dense endpoint mappings while darker reds indicate more dense endpoint mappings. 

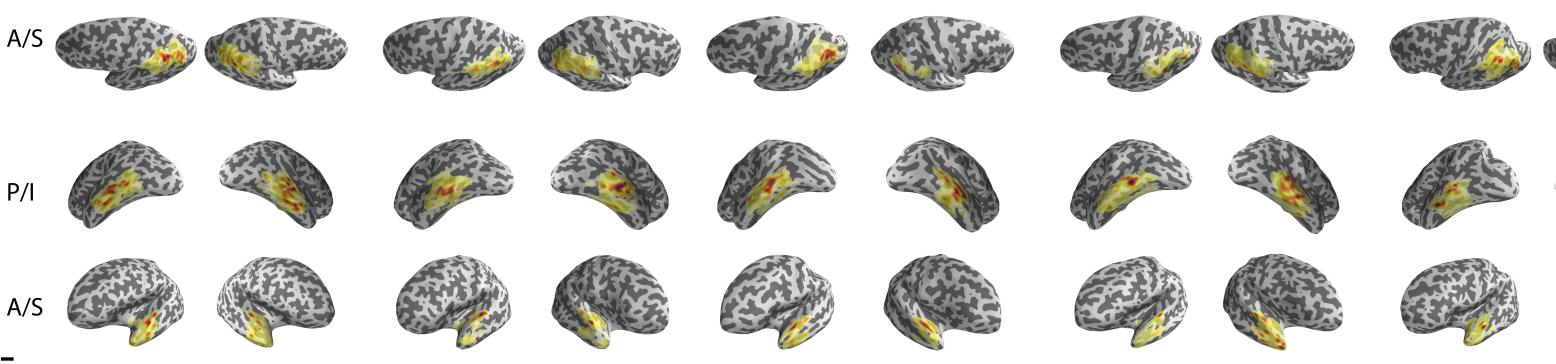

\section{(1)}
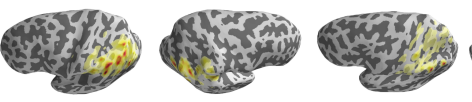

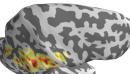
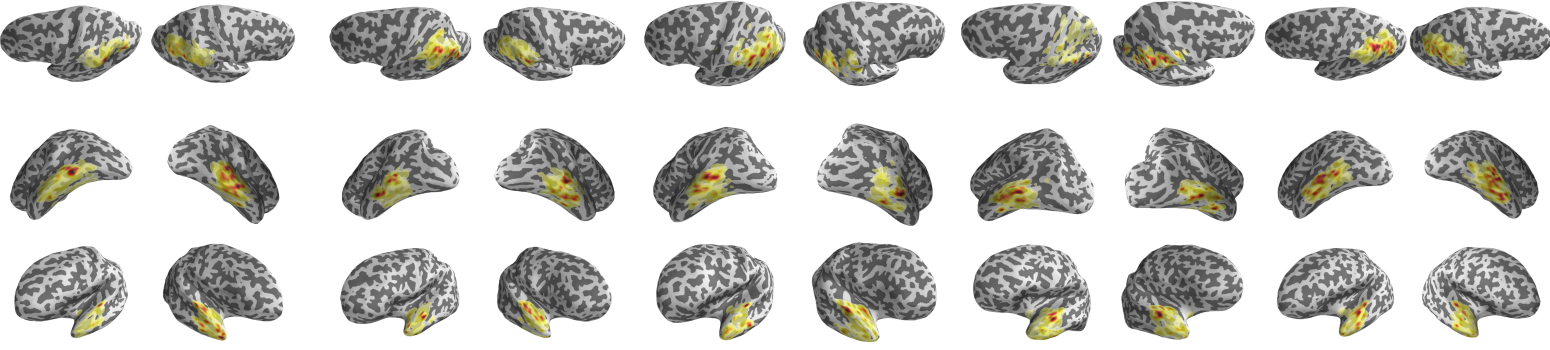

SPL

MdLF-
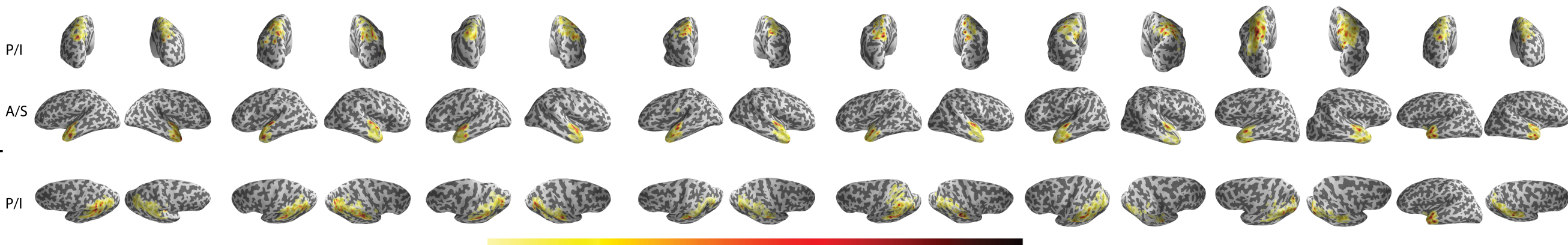

Minimum normalized

Density
Maximum normalized Density

Supplementary Fig. 6. Cortical termination mappings for non-cannonical tracts. Horziontal axis labeling corresponds to subject ID (for HCP, subject numbers; for STN96, subject initials), while L/R indicates hemisphere (L=left, $R=$ right). Vertical axis labeling corresponds to tract name, while $\mathrm{A} / \mathrm{S}$ or $\mathrm{P} / \mathrm{l}$ indicate tract termination group $(\mathrm{A} / \mathrm{S}=$ anterior or superior; $\mathrm{P} / \mathrm{I}=$ posterior or inferior). Heatmaps depicted on cortical surfaces correspond to normalized endpoint density mappings; lighter yellows indicate less dense endpoint mappings while darker reds indicate more dense endpoint mappings. 
$\begin{array}{cc}L & R \\ 21 & \end{array}$

P/I
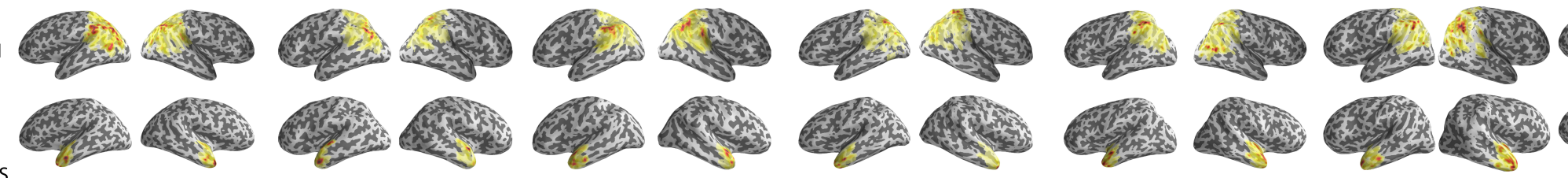

Subj 4

ILF
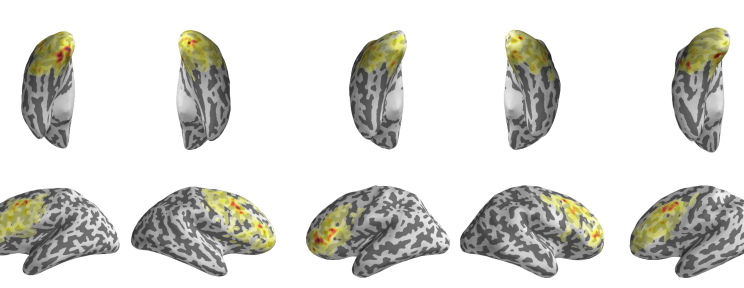

1

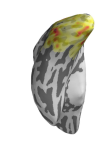

)

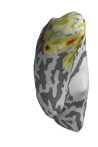

1

.

(1)

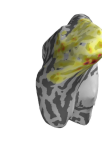

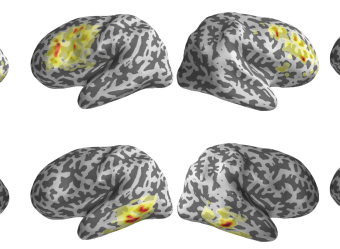

.
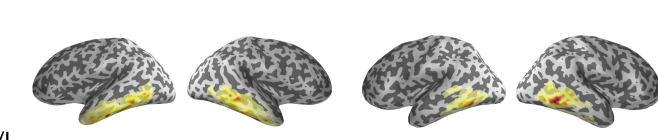

7\%

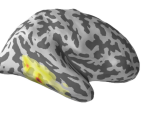

.
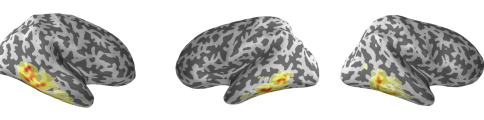

w

(x)

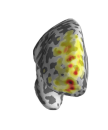

更

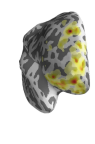

酶
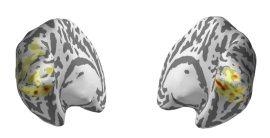

(a)

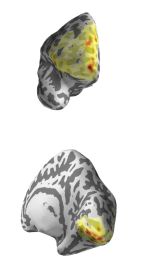

(6)

$\mathrm{R}$

$\frac{\pi}{6}$

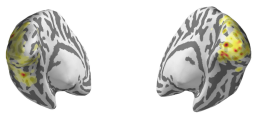

Minimum normalized

Density
Maximum normalized Density

Supplementary Fig. 7. Cortical termination mappings for cannonical tracts. Horziontal axis labeling corresponds to subject ID (for HCP, subject numbers; for STN96, subject initials), while L/R indicates hemisphere (L=left, $R=$ right). Vertical axis labeling corresponds to tract name, while $\mathrm{A} / \mathrm{S}$ or $\mathrm{P} / \mathrm{I}$ indicate tract termination group ( $\mathrm{A} / \mathrm{S}=$ anterior or superior; $\mathrm{P} / \mathrm{I}=$ posterior or inferior). Heatmaps depicted on cortical surfaces correspond to normalized endpoint density mappings; lighter yellows indicate less dense endpoint mappings while darker reds indicate more dense endpoint mappings. 
TPc

pArc

$\mathrm{P} / \mathrm{I}$

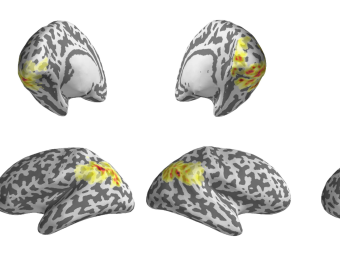

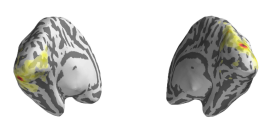

a)

9
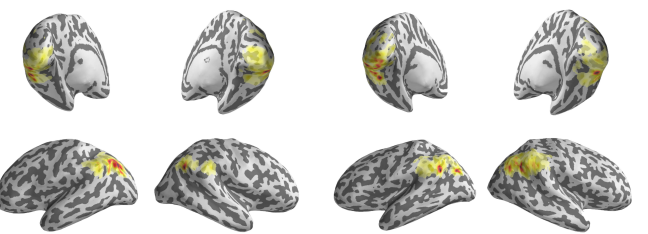
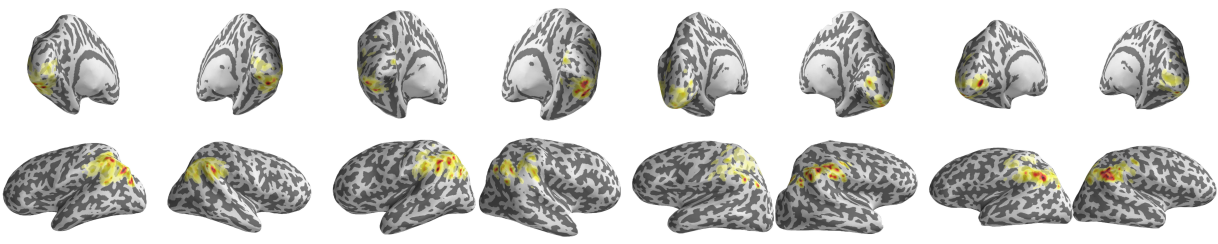

4

(7)
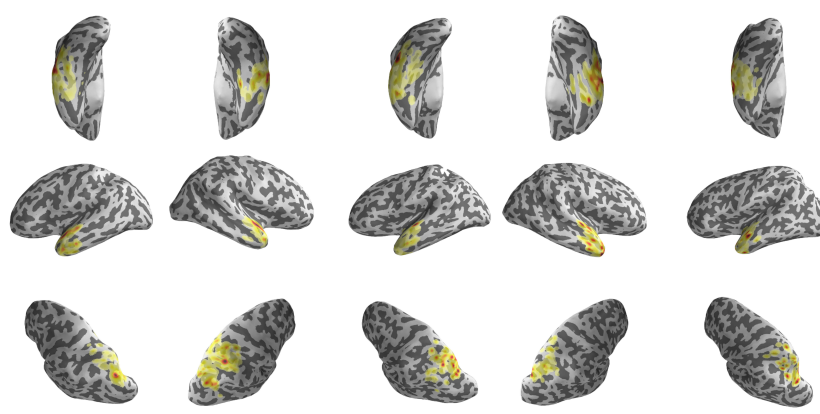

1

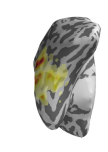

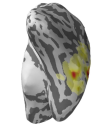

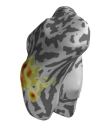

i

(4)

?
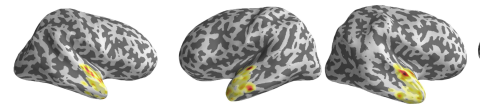

(3)
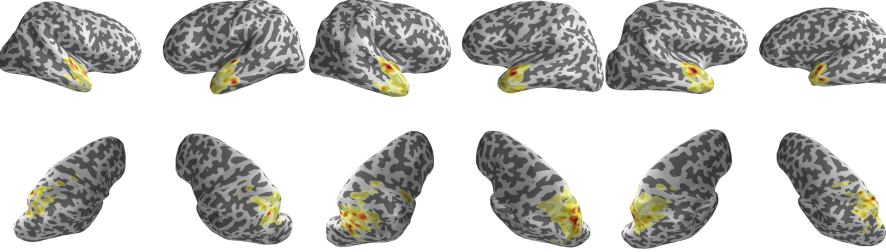

称

实

6

E

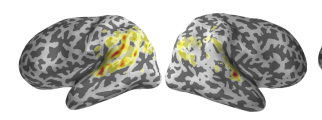

\section{(1)}

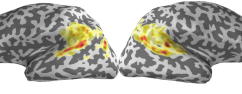

\section{Minimum normalized}

Density
Maximum normalized Density

Supplementary Fig. 8. Cortical termination mappings for non-cannonical tracts. Horziontal axis labeling corresponds to subject ID (for HCP, subject numbers; for STN96, subject initials), while L/R indicates hemisphere (L=left, $R=$ right). Vertical axis labeling corresponds to tract name, while $\mathrm{A} / \mathrm{S}$ or $\mathrm{P} / \mathrm{l}$ indicate tract termination group $(\mathrm{A} / \mathrm{S}=$ anterior or superior; $\mathrm{P} / \mathrm{I}=$ posterior or inferior). Heatmaps depicted on cortical surfaces correspond to normalized endpoint density mappings; lighter yellows indicate less dense endpoint mappings while darker reds indicate more dense endpoint mappings. 


\section{SUPPLEMENTARY REFERENCES}

Caiafa CF, Pestilli F (2017) Multidimensional encoding of brain connectomes

Catani M, de Schotten MT (2012) Atlas of Human Brain Connections (all tracts). In: Atlas of Human Brain Connections. pp 75-238

Catani M, Jones DK, Ffytche DH (2004) Perisylvian language networks of the human brain. Ann Neurol 57:8-16

de Schotten MT, Dell'Acqua F, Forkel SJ, et al (2011) A lateralized brain network for visuospatial attention. Nat Neurosci 14:1245-1246

Gomez J, Pestilli F, Witthoft N, et al (2015) Functionally defined white matter reveals segregated pathways in human ventral temporal cortex associated with category-specific processing. Neuron 85:216-227

Kamali A, Flanders AE, Brody J, et al (2014a) Tracing superior longitudinal fasciculus connectivity in the human brain using high resolution diffusion tensor tractography. Brain Struct Funct 219:269-281

Kamali A, Flanders AE, Brody J, et al (2014b) Tracing superior longitudinal fasciculus connectivity in the human brain using high resolution diffusion tensor tractography. Brain Struct Funct 219:269-281

Lawes INC, Barrick TR, Murugam V, et al (2008) Atlas-based segmentation of white matter tracts of the human brain using diffusion tensor tractography and comparison with classical dissection. Neuroimage 39:62-79

Leong JK, Pestilli F, Wu CC, et al (2016) White-Matter Tract Connecting Anterior Insula to Nucleus Accumbens Correlates with Reduced Preference for Positively Skewed Gambles. Neuron 89:63-69

Makris N, Preti MG, Wassermann D, et al (2013) Human middle longitudinal fascicle: segregation and behavioral-clinical implications of two distinct fiber connections linking temporal pole and superior temporal gyrus with the angular gyrus or superior parietal lobule using multi-tensor tractography. Brain Imaging Behav 7:335-352

Martino J, De Witt Hamer PC, Berger MS, et al (2013) Analysis of the subcomponents and cortical terminations of the perisylvian superior longitudinal fasciculus: a fiber dissection and DTI tractography study. Brain Struct Funct 218:105-121

Martino J, García-Porrero JA (2013) Wernicke perpendicular fasciculus and vertical portion of the superior longitudinal fasciculus: in reply. Neurosurgery 73:E382-3

Pestilli F (2015) Test-retest measurements and digital validation for in vivo neuroscience. Sci Data 2:140057

Pestilli F, Yeatman JD, Rokem A, et al (2014) Evaluation and statistical inference for human connectomes. Nat Methods 11:1058-1063

Rokem A, Takemura H, Bock AS, et al (2017) The visual white matter: The application of diffusion MRI and fiber tractography to vision science. J Vis 17:4

Takemura H, Caiafa CF, Wandell BA, Pestilli F (2016) Ensemble Tractography. PLoS Comput Biol 12:e1004692

Wang Y, Fernández-Miranda JC, Verstynen T, et al (2013) Rethinking the role of the middle longitudinal fascicle in language and auditory pathways. Cereb Cortex 23:2347-2356

Wu Y, Sun D, Wang Y, et al (2016) Tracing short connections of the temporo-parieto-occipital region in the human brain using diffusion spectrum imaging and fiber dissection. Brain Res 1646:152-159 